\title{
STATUS AND PERSPECTIVES OF PHOTOPRODUCTION EXPERIMENTS
}

C. A. HEUSCH

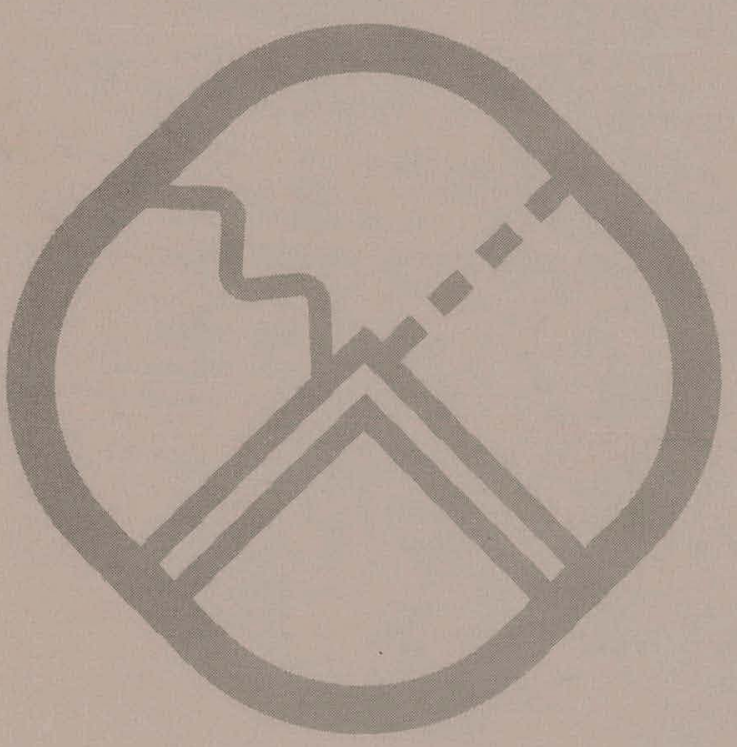

SYNCHROTRON LABORATORY

CALIFORNIA INSTITUTE OF TECHNOLOGY 


\section{DISCLAIMER}

This report was prepared as an account of work sponsored by an agency of the United States Government. Neither the United States Government nor any agency Thereof, nor any of their employees, makes any warranty, express or implied, or assumes any legal liability or responsibility for the accuracy, completeness, or usefulness of any information, apparatus, product, or process disclosed, or represents that its use would not infringe privately owned rights. Reference herein to any specific commercial product, process, or service by trade name, trademark, manufacturer, or otherwise does not necessarily constitute or imply its endorsement, recommendation, or favoring by the United States Government or any agency thereof. The views and opinions of authors expressed herein do not necessarily state or reflect those of the United States Government or any agency thereof. 


\section{DISCLAIMER}

Portions of this document may be illegible in electronic image products. Images are produced from the best available original document. 
Status and Perspectives of Photoproduction Experiments ${ }^{*}$

\section{Lectures Delivered at}

The International School of Physics, "Ettore Majorana", Erice, Sicily July i3-27, 1968

Clemens A. Heusch

California Institute of Technology

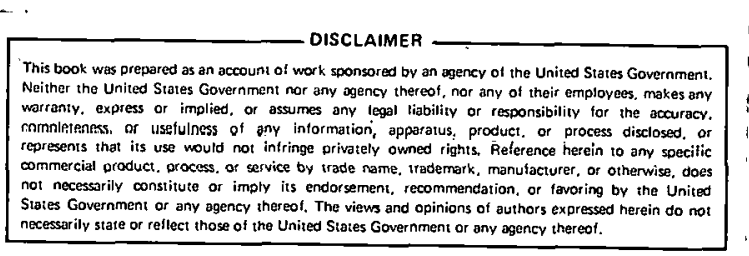

* Work supported in part by the U. S. Atomic Energy Commission. Prepared under Contract AT(11-1)-68 for the San Francisco Operations Office, U. S. Atomic Energy Commission. 
Status and Perspectives of Photoproduction Experiments

In line three lectures presented this year at the Erice Summer School, I shall attempt to give an up-tin-rate picture of what is luw and, to some, exciting in the photoproduction ficld. There are a few severe limitations forced on us by the width of the field and the finite time available. I will therefore assume that the student is acquainted with the general trend of modern particle physics; that he has some knowledge of techniques such as used in partial wave analysis of scattering amplitudes, of the simplest consequencef of the invariance of strong interaction parameters under $\mathrm{SU}(2)$ and $\mathrm{SU}(3)$ transformation, of the hasies of the non-rclativistle quark model, and the concept of Regge poles. But even for strictly data-oriented people, I hope to give a fairly full picture of what has recently been happening in the field of photon-initiated hadronic interactiuns leading to a nucleon-plus-boson f'inal state. No attempt was made to avoid personal bias for particular aspects of the field. We will deal primarily with the photoproduction of single non-strange pseudoscalar and vector mesons - data, problems they pose, and current ideas toward their interpretation. 
Contents

Lecture 1 The Photoproduction of Pseudo-scalar Mesons in the Isobar Region.

1.1 Introduction: Features of Photoproduction Experiments 4

1.2 The Isobar Region in $\pi$ Photoproduction $\quad 7$

1.3 Helicity Formulation of Partial Wave Analysis $\quad 10$

1.4 Sum Rules for Photoproduction Amplitudes 21

1.5 Other Mesons: K and $\eta$ Photoproduction in the Isobar Region 22

1.h Dispersion Treatment $\quad 31$

1.7 Isobars and SU(3) 33

1.8 Isobars in the Non-relativistic Quark Model 36

Lecture 2 The Photoproduction of Vector Mesons.

2.I Models for the Photoproduction of Vector Mesons 39

$2.2 \rho$ Photoproduction $\quad 45$

$2.3 \omega$ Photoproduction $\quad 63$

$2.4 \Phi$ Photoproduction $\quad 68$

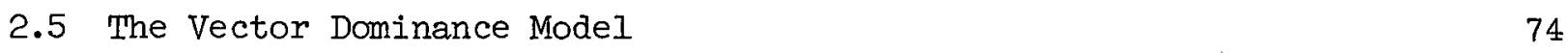

2.6 Some Specific Tests of the Vector Dominance Model 78

2.7 Total Hadronic Cross-section $\sigma_{\text {tot }}(\gamma \mathrm{p})$

2.8 Measurement of the Total Cross-section $\sigma_{\text {tot }}(\gamma \mathrm{p})$.

Lecture 3 The Photoproduction of Pseudo-scalar Mesons at High Energies.

3.1 Data on Forward Photoproduction (t Channel Poles) 92

3.2 Data on Backward Photoproduction (u Channel Folca) 101

3.3 The Simple-rinded Regge Picture 106

3.4 The Not-So-Simple-Minded Regge Picture:Cuts,Fixed Poles,Conspiracy 118

3.4 Interpretation of Forward Dips or Peaks: Conspiracy or Not? 121

3.6 The $\pi^{-} / \pi^{+}$Ratio 126

3.7 A Few Tests of SU(3) Symmetry. 133

3.8 High-Energy $\eta$ Photoproduction 136

3.9 Concluding Remarks $\quad 143$

Acknowledgments $\quad$. 148

References $\quad 149$ 
Lecture 1 Photoproduction of Pseudoscalar Mesons in the Isobar Region.

1.1 Introduction: Features of Photoproduction Experiments.

We will investigate processes of the type

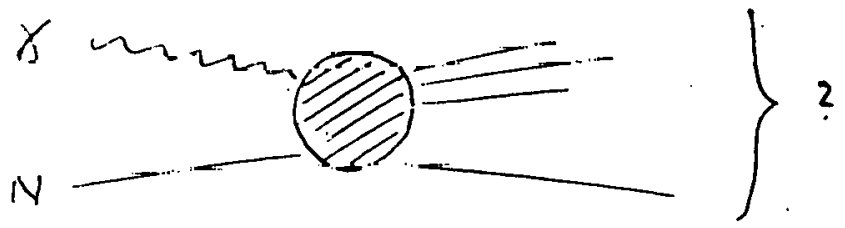

with, to start with, an arbitrary final state. The coupling of the photons to the strong vertex is the distinctive feature. We will, in this context, completely disregard purely electromagnetic interactions. We'll just keep in mind that, as of this time, theorists and experimentalists alike are confident that quantum electrodynamics describes them adequately. We therefore focus our interest on the more problematic field of the strong interactions an coupled lo the plútun-nucleon initial state.

To fully define the incoming channel, let us recall the quantum numbers of the photon:

$$
\begin{aligned}
& J^{P}=I^{-} ; C=-1 \\
& \text { helicity for røal phntinns: } \lambda_{k}=1.1 \\
& \text { helicity for virtual photons: } \lambda_{k}=0, \pm 1
\end{aligned}
$$

We further know that the Gell-Mann-Nishijima formula, $Q=\frac{Y}{2}+I_{3}$, suggests that the photon behaves partly like an isovector, partly like an isoscalar (the hypercharge $Y$ is invariant under transform ations in isospin space). The electromagnetic interactions are 
further known to be invariant under rotations in $U$ spin space, so that we have the SU(2) content of the photon as that of an $a(I=I)+b(I=0)$, and $(U=0)$ object. In SU(3) space, we conventionally view the photon as the $U=0$ member of an octet (an assignment which is yet waiting for unambiguous experimental verlfication of lecture 3).

We notice immediately that, since we have focused on hadronic interactions, we find several hadrons with quantum numbers similar to those of the photon. These are the vector mesons, $0^{0}, \omega, \Phi$. This similarity of space-time properties stimulated the idea of describing the hadronic electromagnetic field in terms of the vector meson fields. We will therefore attempt to tie up the vertices

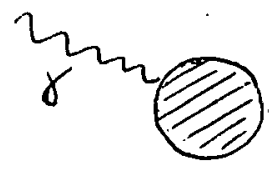

and

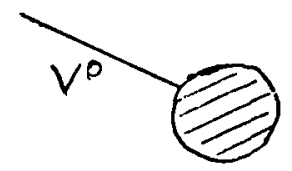

with a possible direct coupling of the type uno $\gamma_{v}^{\gamma} v^{0}$. If such a direct coupling exists, then we may have a chance to describe $\gamma$-hadronic interactions in terms of purely hadronic interactions; we will just have to combine properly the corresponding isospin states of $\mathrm{V}$-mesonic interactions; the coefficients will be related to the photon-vector meson coupling constants $\gamma_{\rho}, \gamma_{\omega}, \gamma_{\Phi^{*}}$ This aspect will be explicltly dealt with in the second lecture; its fundamental importance appears obvious.

Let us turn, for a minute; to experimental aspects; how can we perform $\gamma$-hadronic experiments such that they are of the type of $V$-hadronic reaction studies? 
There are experimental limitations: the photons are available only as secondary beams. Iike all neutral secondary beams, they have the disadvantage of being non-focusable, and, in general, their momentum is not defined. Worse than neutral kaon or neutron beams, the1r masslessness forbids the identification of spectral components by time-of-flight techniques. This means then that

1. Spatial definition is possible only through collimation (meaning intencity loss aud some linavoidable production of secondaries);

2) Momentum definition, short of kinematical reconstruction from the final state, is effected only through fairly tricky techniques. They yield low intensities (as in "photon tagging", because of thin radiator requirements), and mostly degrade the obtainable maximum photon energy (as in positron annihilation, bremsstrahlung production from crystale, and backward compton scattering of lasser photons). Unavoidable backgrounds and collimation problcms allu to the aifficulty.

3) For the definition of polarization components, the absence of a photon magnetic moment excludes direct methods. Crystal bremsstrahlung and scattered laser photons can be used, but there is always a price to pay, as above. For virtual photons, definite polarization states are available in electron- ne mun-In1tiated reactions.

Worst of all, non-hadronic processes see to it that the experiment, if not warefully and ingeniously defined, may be swamped by backgrounds which stem from the abundance of final-state particles due to purely electromagnetic processes (pair production, Compton effect) in the target and along the beam line. 
1.2 The Isobar Region in $\pi$ Photoproduction.

If we take a look at the total cross section data for the photoproduction of single pions below a total energy of $\sim 2 \mathrm{GeV}$, (Fig. 1.2-1), a pronounced resonance structure suggests that the production may proceed dominantly through resonant intermediate states. We might therefore try to interpret the experimentally observed phenomena in terms of diagrams of this tyoe,

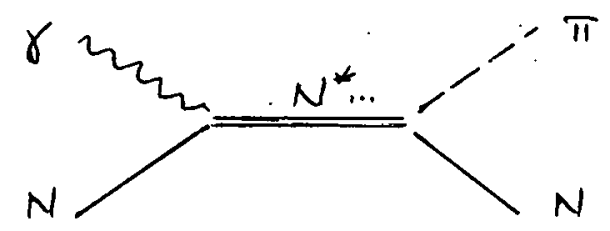

(where the photon, at the left vertex, couples either to the charge or to the magnetic moment of the nucleon). Fig. 1.2-2 shows that the differential cross-sections at $0^{\circ}$ and $180^{\circ}$ do not exhibit the same structure as the total cross-section: We will therefore look into terms which will be present in addition to these s-channel poles. The Born diagrams

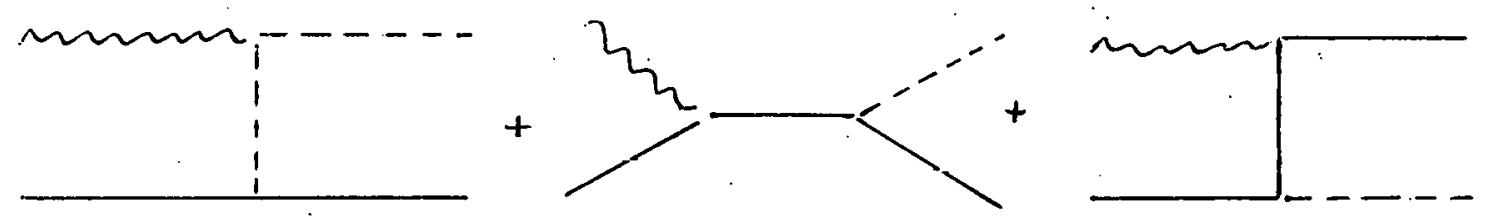

associated with the lowest-mass poles in the $s, t$, and u-channels, are obvious candidates. The first diagram (the $\pi$ pole) will exist for charged $\pi$ production only (c conservation forbids the vertex 


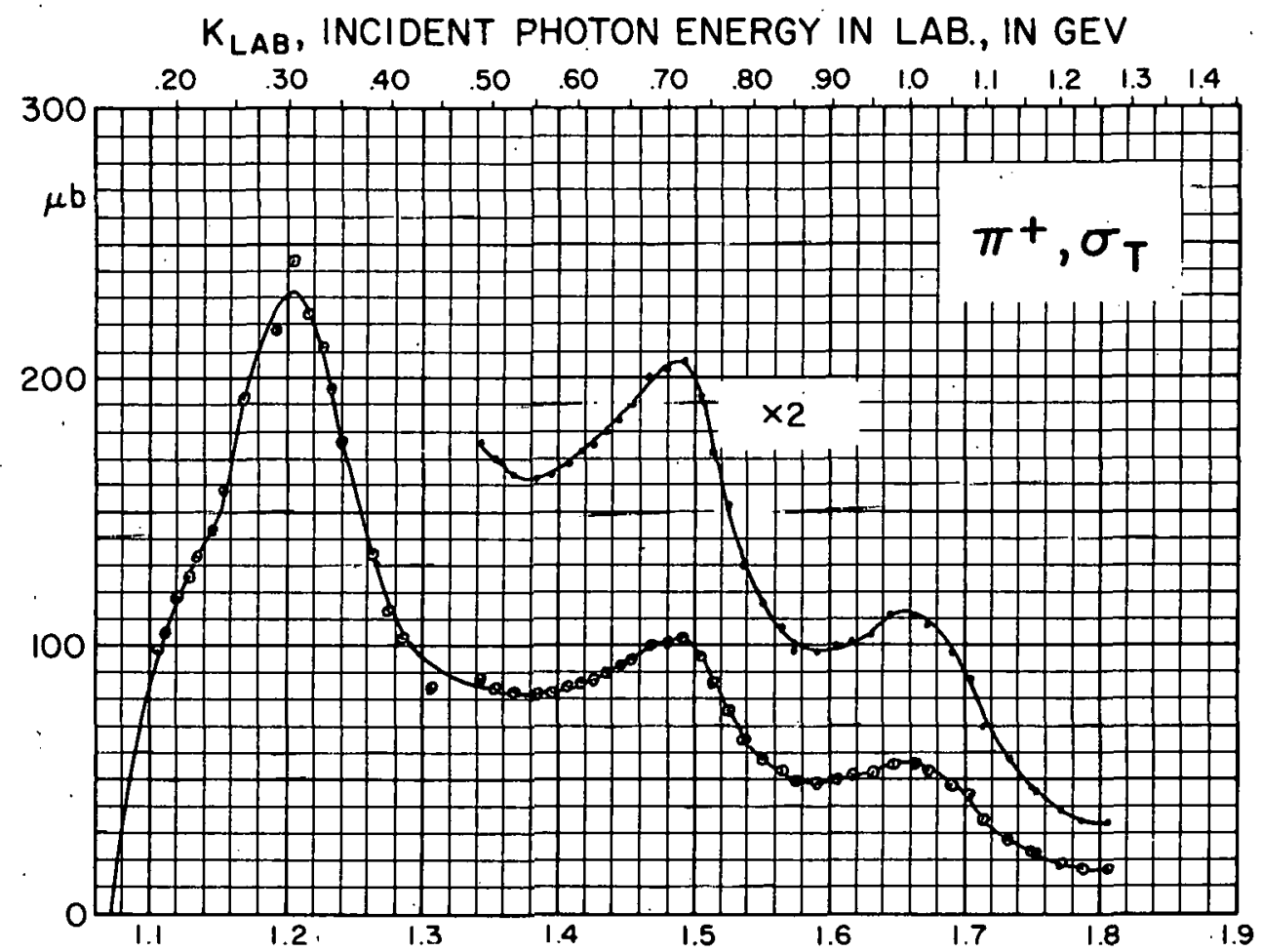

Fig. 1.2-1 Total cross-section for $\gamma p \rightarrow \pi^{+} n$ from threshold

) to $\mathrm{E}_{\gamma}=1.4 \mathrm{GeV}$. (From Ref. No. 7)

\section{$K_{\text {LAB }}$, INCIDENT PHOTON ENERGY IN LAB., IN GEV}

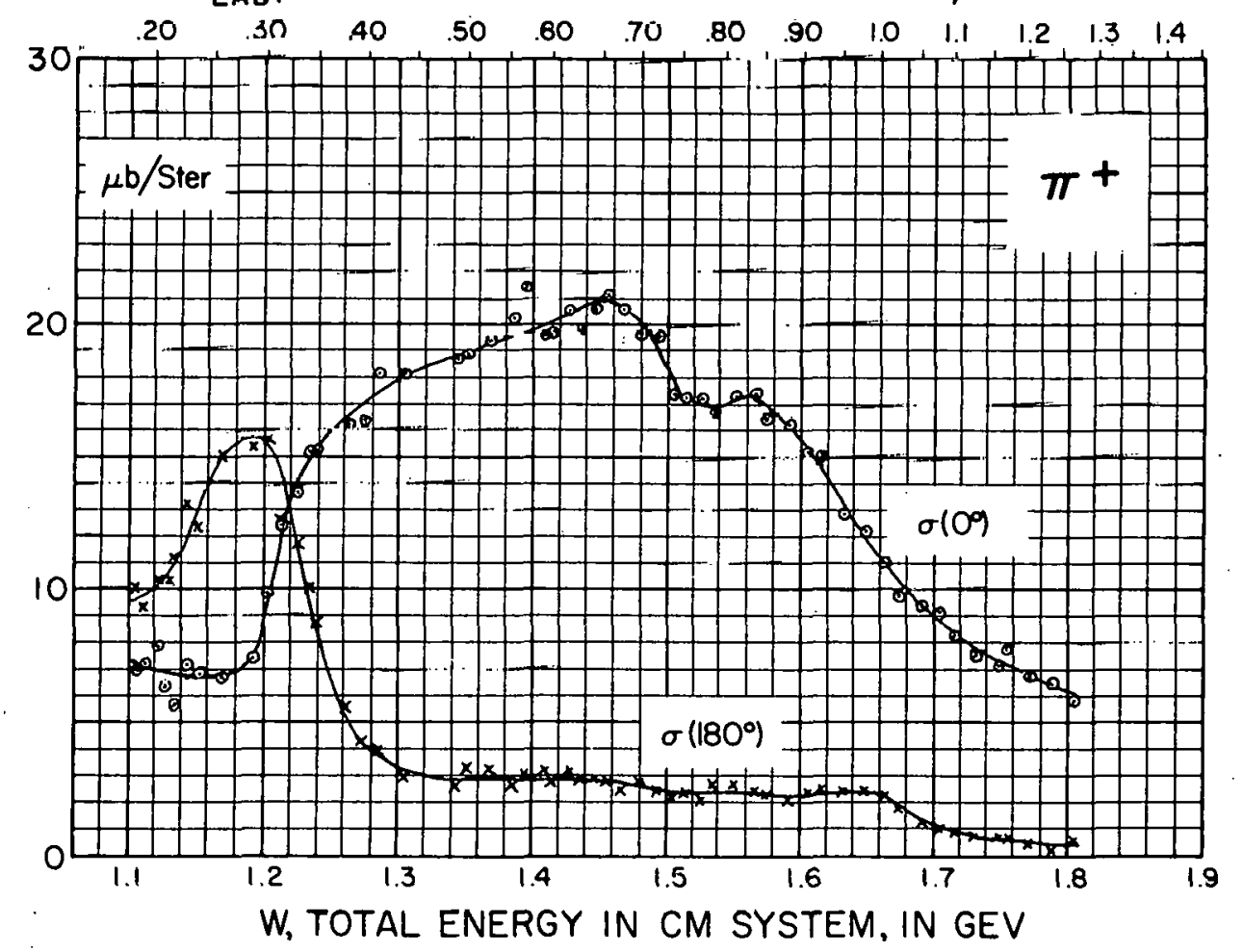

Fig. 1.2-2 Differential cross-section for $\gamma \mathrm{p} \rightarrow \pi^{+} \mathrm{n}$ at $0^{\circ}, 180^{\circ}$. (From Ref. No. 7) 
$\left.\gamma \pi^{\circ} \pi^{\circ}\right)$. The photon coupling to the meson current will give a contribution of the form $\vec{\epsilon} \cdot \vec{q}(\vec{\epsilon}=$ photon polarization, $\vec{q}=\pi$ momentum $)$. When averaged over photon polarizations, it will give a contribution to the cross-section of the form

$$
\frac{d \sigma}{d \Omega} \sim e^{2} \sin ^{2} \theta \vec{q}^{2}
$$

i.e., it will not influence the $0^{\circ}$ or $180^{\circ}$ cross-sections. Since we need a forward and backward contribution to the cross-section, we add the second diagram (direct channel nucleon pole, whose addition is necessary to make the "Born approximation" gauge invariant) and, possibly, the third (crossed or u-channel nucleon pole).

$s$ channel poles are characterized by quantum numbers $\mathcal{J}, I ; m, \Gamma$. They show up as resonance-like behavior in one particular partial wave and may therefore lead to a typical angular structure in the cross-section. t- and u-channel poles, on the other hand, when projected into the $s$ channel, will lead to many multipoles contributing to the angular distribution.

For the $\pi$ exchange diagram, where the small-t behavior is close to the pole at $\cos \theta=\frac{1}{\beta_{\pi}} \cdot\left(\beta_{\pi}=\pi\right.$ velocity $)$, i.e., close to the exchange of a physical particle, a large number of multipoles are expected to contribute to the amplitude $A$.

$$
A_{\pi \operatorname{exch}} \sim \frac{1}{1-\beta_{\pi} \cos \theta} \sim \frac{1}{t-m_{\pi}^{2}}
$$

For $\cos \theta \approx 1, \beta_{\pi} \approx 1$, many $\ell$ waves will be involved; the forces are long-range. To still find the effect of isobars in the pressence of this pnl.e term, the recipe of Moravcsik ${ }^{(1)}$ is to divide out the $\pi$ pole, 
then expand in terms of powers of $\cos \theta$

$$
\left(1-\beta_{\pi} \cos \theta\right)^{2} \frac{d \sigma}{d \Omega}=\sum_{n} c_{n} \cos ^{n} \theta
$$

in order to find angular characteristics, which may then be correlated with individual resonant s-channel contributions.

\subsection{Helicity Formulation nf Partial Wave Alulys1s.}

"Isobar models", consisting of the analysis of photoproduction data in terms of the diagrams mentioned above in the framework of some pragmatic fitting procedure, have been increasingly successful in the phenomenological interpretation of experimental material below $\sim 2 \mathrm{GeV}$ in recent years. Gourdin and $\operatorname{Salin}^{(2)}{ }^{(2)} \operatorname{Salin}^{(3)}, \operatorname{Beder}^{(4)}$, and, most recently, Wa.7ker, ${ }^{(5)}$ have bccil allle to reproduce experimentally observed features well. with such models. (See also Chau et. al. )

Let uis stop briefly and give the relevant. formulatiun in terms of the helicity langruage as spelled out by Jacob and Wick (6), which has proven to be particularly suited to the problem.

We quantize the spins in the initial and final states of the interaction along the directions of photon and pion momenta $\mathrm{k}$ and $\mathrm{q}$, respectively, for the process $\gamma \mathbb{N} \rightarrow \pi \mathbb{N}$, in the cenlur-mass system. We can then def'ine the helicity amplitudes

$$
A_{\mu, \lambda}(\theta, \Phi) \text {, with } \mu=\lambda_{q}-\lambda_{2}=-\lambda_{2} \text {, and } \lambda=\lambda_{k}-\lambda_{1}
$$

the final and initial-state helicities $\left(\lambda_{1}, \lambda_{2}\right.$ refer to the corresponding nucleon helicities). For real, transverse photons, $\lambda_{k}= \pm 1 . \lambda$ then has four possible values; $\pm 1 / 2, \pm 3 / 2$; with $-\lambda_{2}=\mu$ 
being $\pm 1 / 2$, we have a chọice of 8 helicity amplitudes. However, parity conservation provides a link between the $\lambda_{k}=+1$ and -1 amplitudes.

$$
A_{-\mu,-\lambda}(\theta, \Phi)=e^{i(\lambda-\mu)(\pi-2 \Phi)} A_{\mu, \lambda}(\theta, \Phi)
$$

so that we are left with four independent amplitudes; we choose $\lambda=+1$, and restrict ourselves to the plane $\Phi=0$; we then obtain the "helicity amplitudes"

$$
\mathrm{H}_{1} \cdots \mathrm{H}_{4}(\theta)=\mathrm{A}_{\mu \lambda}(\theta, \Phi=0) ; \lambda_{\mathrm{k}}=+1 \text {. }
$$

In terms of these amplitudes, we write down the experimentally observable quantities. The differential cross-section is

$$
\frac{d \sigma}{d \Omega}(\theta)=\frac{1}{2} \frac{\mathrm{q}}{\mathrm{k}} \sum_{i=1}^{4}\left|\mathrm{H}_{i}\right|^{2}
$$

The polarization of the final-state nucleon in the direction $\vec{q} \times \vec{k}$ is then (we denote the differential cross-section briefly by $\sigma(\theta)$ )

$$
P(\theta)=\frac{q}{k} \frac{l}{\sigma(\theta)} \operatorname{Im}\left(H_{1} H_{3}^{*}+H_{2} H_{4}^{*}\right)
$$

If we have a polarized nucleon target, we can define the asymmetry parameter $T(\theta)=\frac{\sigma_{+}{ }^{-\sigma_{-}}}{\sigma_{+}+\sigma_{-}}$, with $\sigma_{+}, \sigma_{-}$the differential cross-sections for target polarization up or down with respect to $\overrightarrow{\mathrm{k}} \times \vec{q}$. Then, we can express

$$
\mathrm{T}(\theta)=\frac{\mathrm{q}}{\mathrm{k}} \frac{\mathrm{l}}{\sigma(\theta)} \mathrm{Im}\left(\mathrm{H}_{1} \mathrm{H}_{2}^{*}+\mathrm{H}_{3} \mathrm{H}_{4}^{*}\right)
$$

Last, we define an asymmetry parameter $\Sigma(\theta)=\frac{\sigma_{\perp}-\sigma \|}{\sigma_{\perp}+\sigma \|}$ for 
processes initiated by polarized photons, where the $\sigma_{\perp}, \sigma_{\|}$ are the cross-sections for photon polarization perpendicular and parallel to the production plane. We find

$$
\Sigma(\theta)=\frac{\mathrm{q}}{\mathrm{k}} \frac{\mathrm{l}}{\sigma(\theta)} \operatorname{Re}\left(\mathrm{H}_{1} \mathrm{H}_{4}^{*}-\mathrm{H}_{2} \mathrm{H}_{3}^{*}\right)
$$

Determination of all these observable parameters would lead to a more complete picture; however, final-state nucleon polarization $\mathrm{P}$ and. polarized photon asymmetry $\Sigma$ have been measured only at a few angles and energies, $(7)$ and polarized nucleon targets suited to photoproduction work are only now starting to be practically considered ${ }^{(8)}$. The polarized proton targets used in $\pi$ p scattering experiments up to now, contained heavy elements which will not allow for a reconstruction of the full kinematics in the presence of a continuous incoming photon spectrum. Even the existing cross-section data are by no means as complete and consistent as one would wish; particularly the photoproduction processes

$$
\begin{aligned}
& \gamma \mathrm{n} \rightarrow \mathrm{n} \pi^{\circ} \\
& \gamma_{\mathrm{n}} \rightarrow \mathrm{p} \pi^{-}
\end{aligned}
$$

have been investigated only sparsely. (7).

To decompose the observables into partial-wave contributions, we now write down the partial-wave expansion for the helicity amplitudes

$$
A_{\mu \lambda}(\theta, \Phi)=\sum_{j} A_{\mu \lambda}^{j}(2, i+1) d_{\lambda \mu}^{j}(\theta) e^{i(\lambda-\mu) \Phi}
$$


For our specific case, with $\Phi=0$, we have

$$
H_{i}(\theta)=\sum_{j} H_{i}^{j}(2 j+1) d_{\lambda_{\mu}}^{j}(\theta)
$$

The $A_{\mu}{ }_{j}^{j}$ and $H_{i}^{j}$ are the helicity coefficients, for given helicity and angular momentum; the angular functions are mutually orthogonal and appropriately normalized when integrated over the solid angle. In order to have helicity coefficients corresponding to states of definite parity, we have to take sums and differences of final states having opposite helicity, $+\mu$ and $-\mu$, in order to define

$$
\begin{aligned}
& A_{n+}=-\frac{1}{\sqrt{2}}\left(A_{\frac{1}{2} \frac{1}{2}}^{j}+A_{-\frac{1}{2}, \frac{1}{2}}^{j}\right) \\
& A_{(n+1)-}=\frac{1}{\sqrt{2}}\left(A_{\frac{1}{2}}^{j} \frac{1}{2}-A_{-\frac{1}{2}, \frac{1}{2}}\right) \\
& B_{n+}=\sqrt{\frac{2}{n(n+2)}\left(A_{\frac{1}{2}}^{j} \frac{3}{2}+A_{-\frac{1}{2}} j_{\frac{2}{2}}\right)} \\
& B_{(n+1)-}=-\sqrt{\frac{1}{n(n+2)}}\left(A_{\frac{1}{2}}^{j} \frac{3}{2}-A_{-\frac{1}{2}} \frac{j}{2}\right)
\end{aligned}
$$

The $A$ and $B$ are seen to refer to initial helicities $\frac{2}{2}$ and $\frac{3}{2}$, respectively. The subscript notation, with $n=j-1 / 2$, is that used in the CGLN formalism $(9)$. The brackets are the same as $\left(\mathrm{H}_{2}^{j} \pm \mathrm{H}_{4}^{j}\right),\left(\mathrm{H}_{I}^{j} \pm \mathrm{H}_{3}^{j}\right)$. It is then straight-forward to express the four independent helicity amplitudes in terms of these coefficients $A, B$ and the angular functions introduced in (1.3-8).

It is interesting to look at the angular structure of a few of the helicity amplitudes, as they are influenced by the "hellclty elements" A, B from eq. (1.3-10). Fig.1.3-1. shows the dependence 

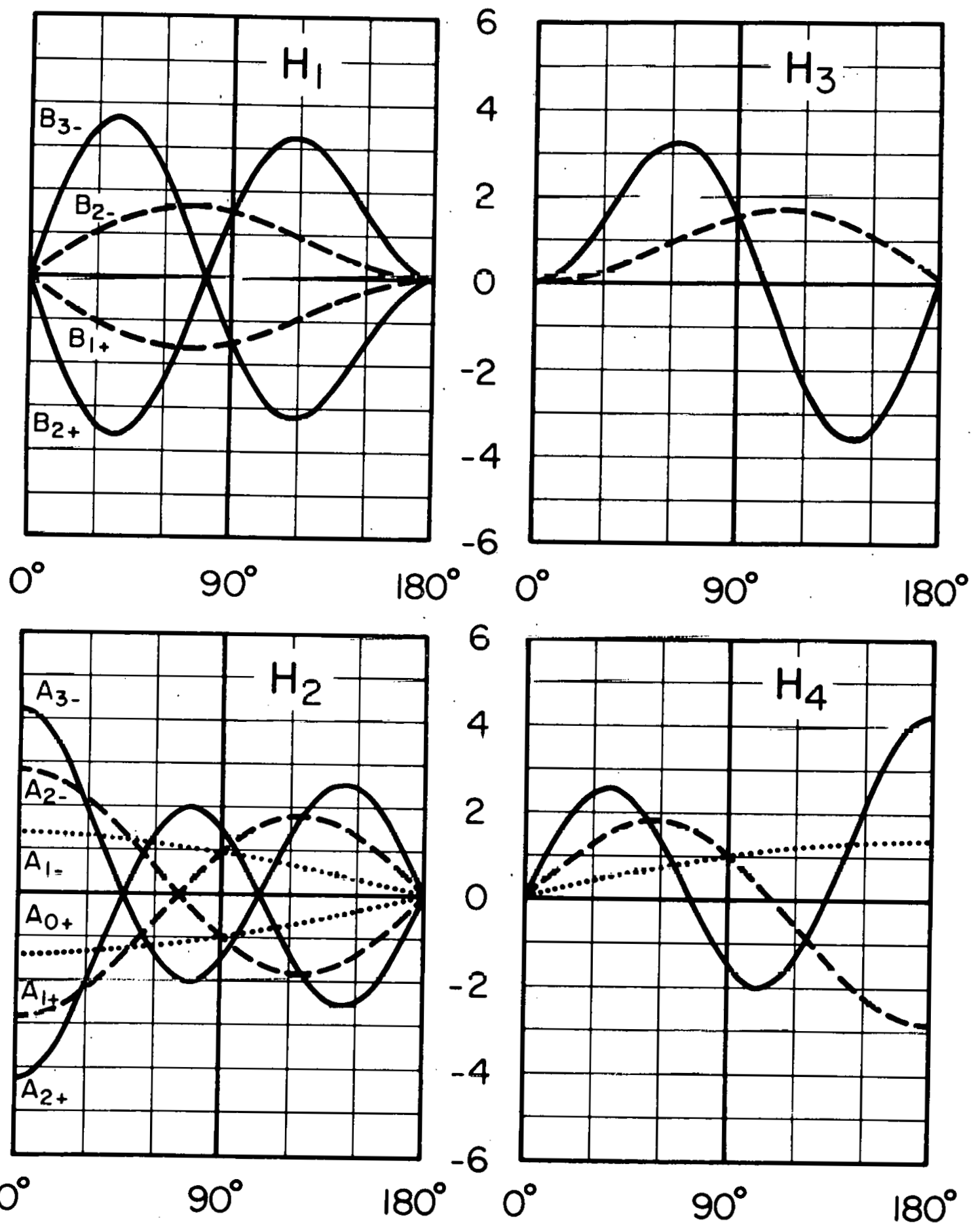

Fig. 1.3-1 Influence of the helicity elements A, B on the helicity amplitudes $H_{1} \ldots H_{4}$, for $j \leq \frac{5}{2}$ (From Ref. No. 5). 
of $\mathrm{H}_{1} \cdot-\mathrm{H}_{4}$ on the individual $A^{\prime} \mathrm{s}$ and $B^{\prime} \mathrm{s}$ for $j \leq \frac{5}{2}$. Two features stand out: with increasing $j$, the angular behavior gets more complicated (as. we expect); and the forward and backward amplitudes each show contributions from one helicity amplitude only ( $\mathrm{H}_{2}$, and $\mathrm{H}_{4}$, respectively). This points up a particularly useful feature of the formalism employed.

Using the helicity language, $R$. Walker ${ }^{(5)}$ at CalTech has recently finished an extensive phenomenological study of single $\pi$ photoproduction data from many laboratories. He fits the data for all isospin amplitudes as far as they are experiwentally accessible, in terms of a model incorporating Born terms (electric coupling only); s-channel resonances as known from $\pi N$ phase shift analysis; and a non-resonant background in the low partial waves which is required to vary smoothly with energy. Resonances are inserted in a Breit-Wigner form with an appropriate phase-space factor.

Notwithstanding the incompleteness of the data and the obvious Iimitations of the model, the method is fairly powerful. Resonances show up when the real or imaginary parts of a particular partial wave amplitude demonstrate a typical behavior with energy like the one in Fig. 1.3-2. Since the $\pi^{+}$data are the most accurately known, we show a few examples of Walker's fits in Fig. 1.3-3.

We now revert to the general picture of section 1.2 , and ask which features of photoproduction in the isobar region have emerged from such analysis. 


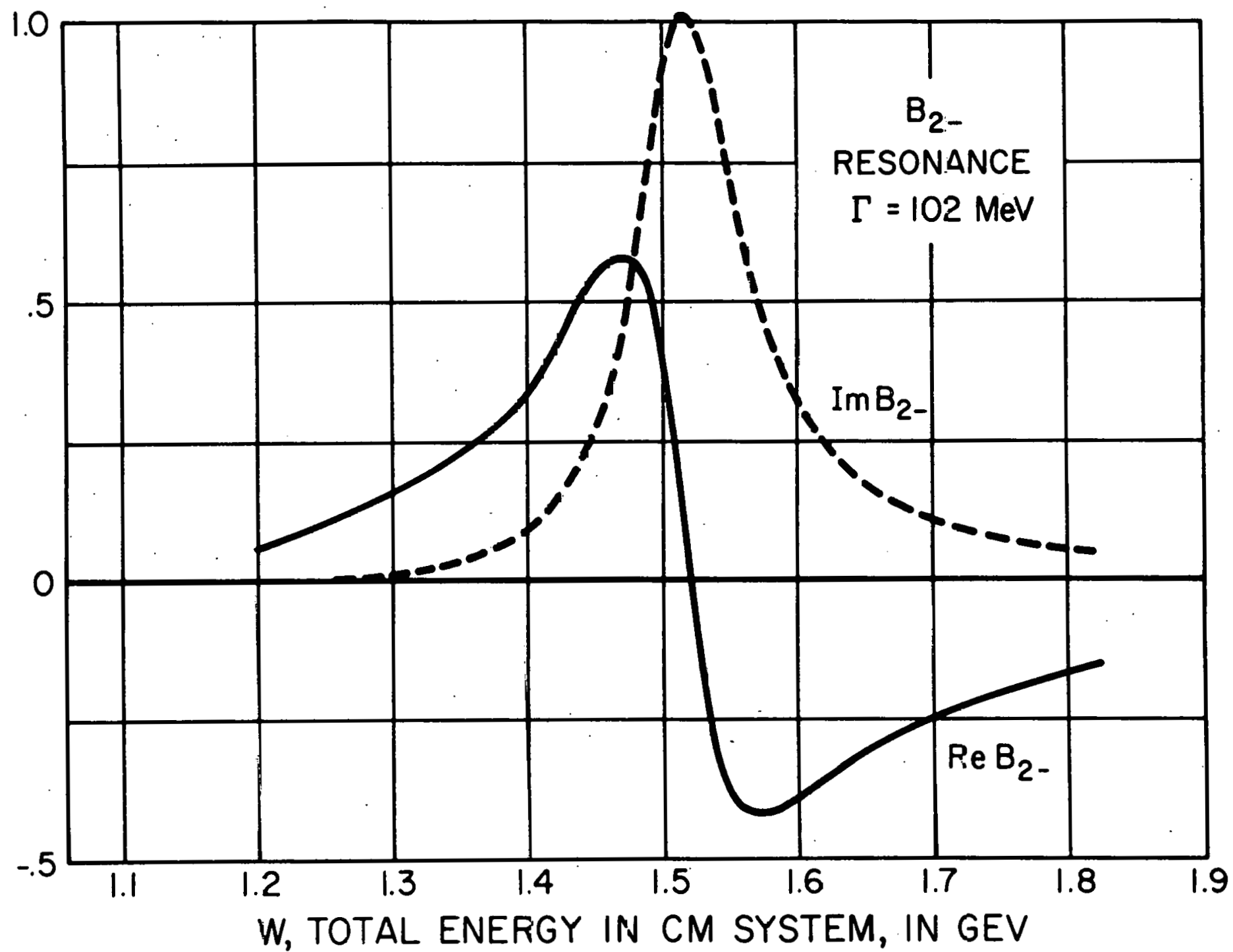

Fig. 1.3-2 Typica $\mathrm{I}$. at resonarce peak of $D_{13}$ (1520). (From Ref. No. 5) 

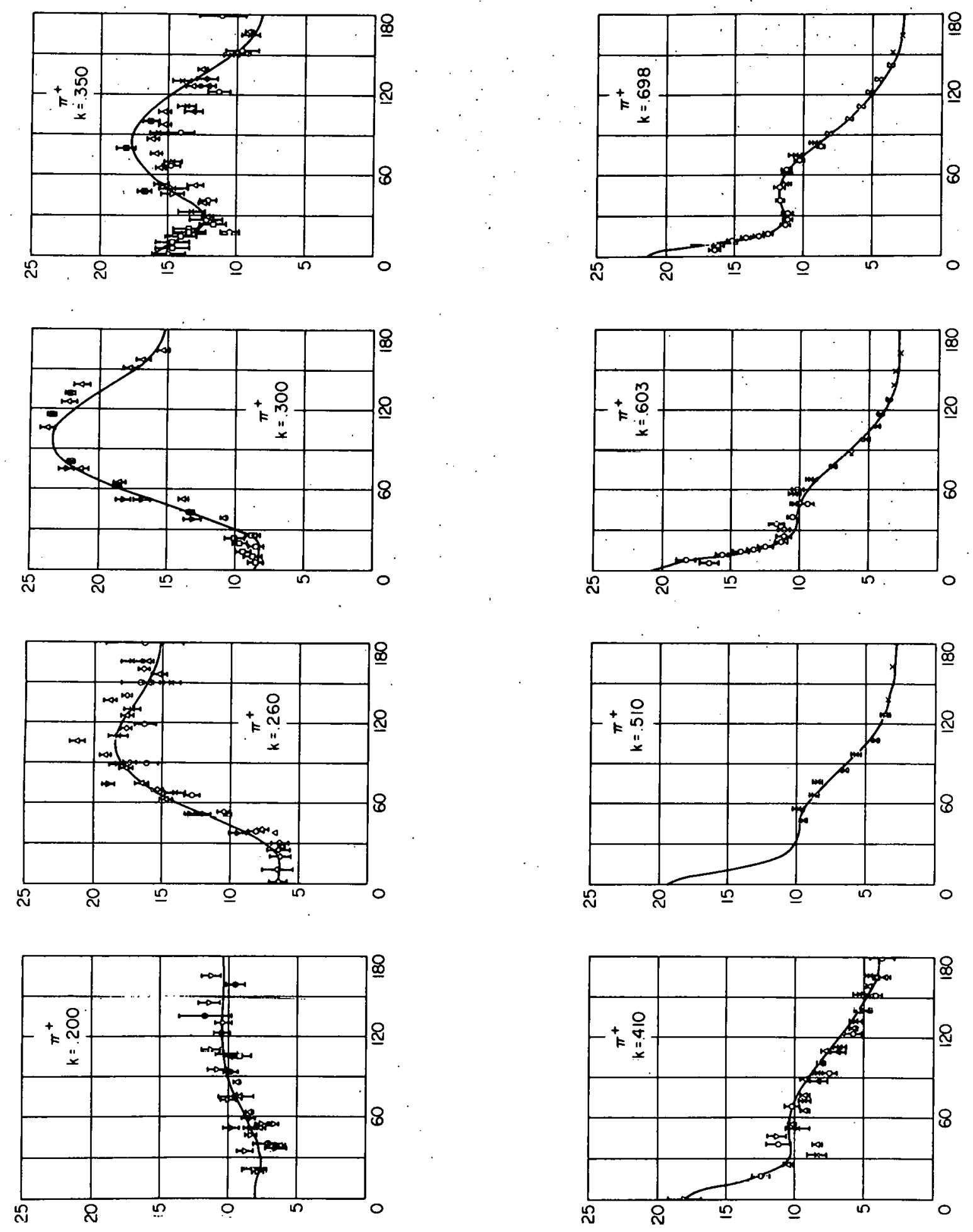

Fig. 1.3-3 Examples of Isobar Model Fits to $\pi^{+}$Production Lata. From Ref. No. 5) 
I) It is :confirmed that the "first resonance" ( $\Delta$ 1238) is predominantly excited by the magnetic dipole (Fig. 1.3-4 shows, for $\pi^{\circ}$ photoproduction, the relevant $\mathrm{H}_{i}$ 's; the dominant $90^{\circ}$ contribution comes from the imaginary part of $\left.\mathrm{B}_{1+}\right)$. The resulting strength for this photo-excitation is close to that given by the quark model or by $\mathrm{SU}(6)$ (cf. section 1.8). There is a small admixture ( G\%) of electiris quarrnpolo exoitation, found ftum a cumparison of $0^{\circ}$ and $180^{\circ}$ data with the $90^{\circ}$ cross-section.

2) The $D_{13}$ (1527), the old "second resonance", is produced mainly in the $\mathrm{B}_{2-}$ amplitude; the $\mathrm{F}_{15}$ (1688), the old "third resonance", predominantly in $B_{3-}$. This implies they are both produced from initial helicity 3/2. The absence of $\mathrm{A}$ amplitudes (initial helicity $1 / 2$ ) is reflected in the small effect ma.de hy these states on the $0^{\circ}$ and $180^{\circ}$ cross sections (cf. Fig. 1.?-2). (Remember that angular momentum conservation demands that at these angles, only helicity $1 / 2$ can determine the s-channel behavior.

3) The $S_{1 I}$ (1570) isobur, whlukl 1s likely to produce the $\eta \mathbb{N}$ enhancement close to the threshold for this final state, is indicaled in the $A_{0+}$ amplitude (cf. section 1.7).

4) There is little if any evidence for the production of the $\mathrm{D}_{15}$ (1670). Improvement of polynomial fits in this energy region by lise addition of a small amount of $D_{15}$ was reported earlier by Ecklund and Walker ${ }^{(10)}$ for $\pi^{+}$photoproduction, but the corresponding amplitude $\mathrm{B}_{2+}$ is very small (cf. section 1.8). 

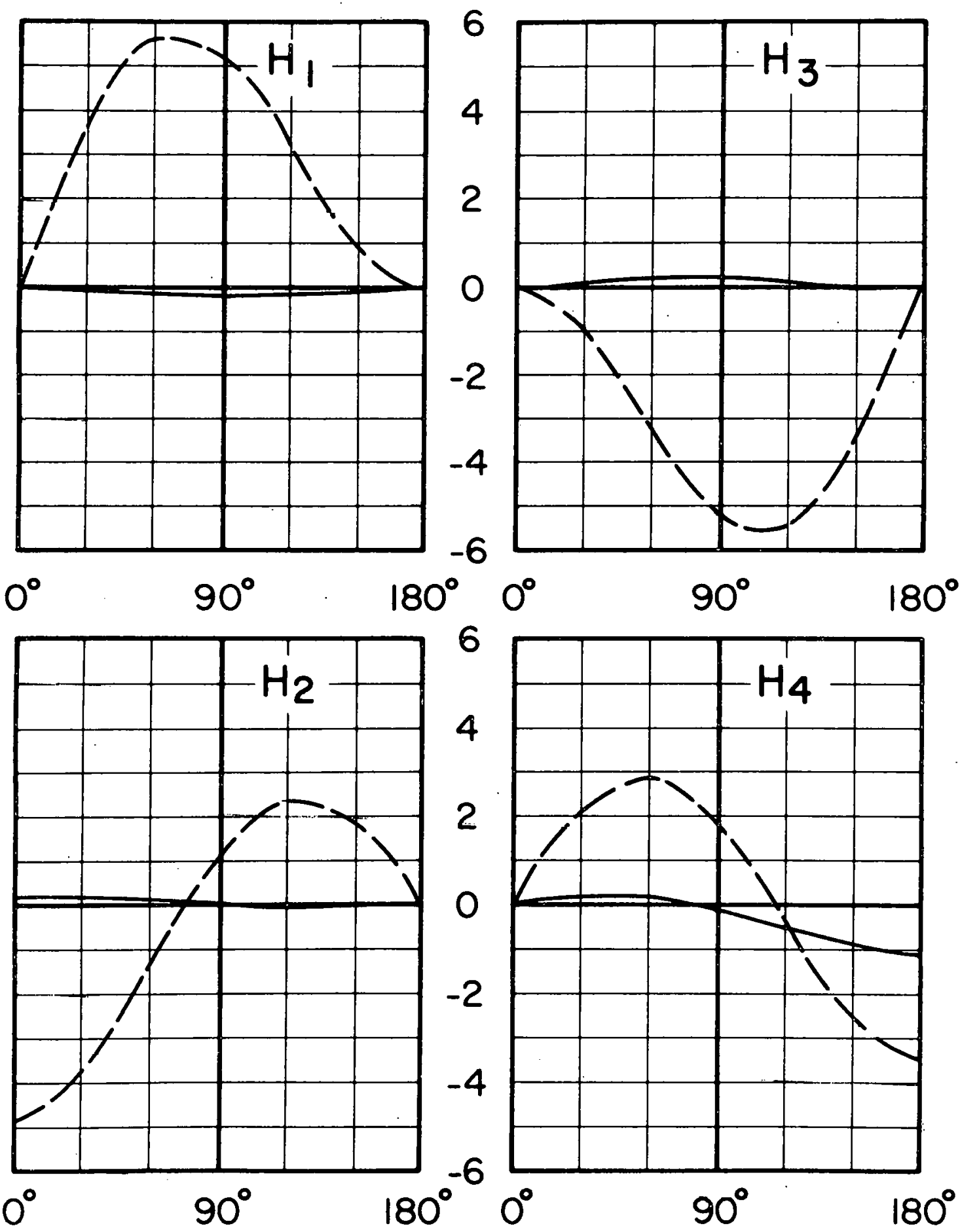

Fig. 1.3-4 Best fits for helicity amplitudes at position of $\Delta$ (1238). Comparison with Fig. 1.3-1 shows that, for the positiveparity state, $\mathrm{B}_{1}$ is the dominant mode. A $\mathrm{A}_{1+}$ admixture shows up at $0^{\circ},{ }^{+} 180^{\circ}$. (Solid curves: ${ }^{1+}$ real part; dashed curves: imaginary part). (From Ref. No. 5). 
5) The $\mathrm{S}_{11}$ (1709) does not emerge in the photoproduction of $\pi$ 's. This may be due to the nature of the fitting procedure employed, and to the fact that the state lies close in energy to other. resonant states. We will see (cf. 1.7) that in the photoproduction of $\eta$ mesons we may have a better chance to investigate this question quantitatively.

6) Similarly, the $\mathrm{P}_{11}$ (1400), the "Koper resonance", has not been uniquely identified in the photoproduction of single $\pi^{\prime}$ s.

7) Most isobars appear to be produced predominantly by isovector photons. Clearly, to make this statement firm, we will need more data (especially from $\pi^{-}$production); and we will need new ideas to understand their implications. Many of the features emerging here have interesting implicatiuns in terms of symmetry considerations and the quark model. We will deaj. with them in Eeotions 1.7 and 1.8 .

Extension to Higher Energies

Phenomenological models of the type discussed have been successful for total energies up to $\sim 1.7 \mathrm{GeV}$. Beyond that range, too many partial waves will have to be considered, and irlerpretätion becomes liss stringent. Also, exchange graphs of the tiyne

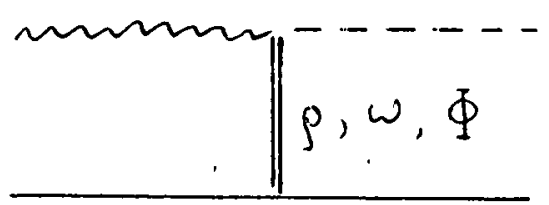

can probably no. longer be disregarded. One attempt to include such exchanges was made by Bloom et. al. (1l), who found that a reggeized vector meson exchange (one can safely assume $\omega$ exchange only) 
considerably improves their fit to cross-section and polarization data in the intermediate energy region. Elementary vector exchange does not fit. In their fit, isobars up to the $F_{I 7}$ (1920) were included, and the trajectory exchange was slowly "turned on" in an empirical way (with the residues assumed to be constant along the trajectory in the $t$ range covered).

We will treat the Regge picture in lecture 3 and remark here only that we will need more, and more precise experimental information before we will be able to link up intelligently the isobar region and the region which is more successfully described in terms of $t$ channel singrlarities.

\subsection{Sum Rules for Photoproduction Amplitudes}

Various sum rules have been proposed in recent years to test physical assumptions, particularly the ideas of current algebra and super-convergence relations. It is often hard to check these against experiment due to a lack of precise information about amplitudes which are hard to extract from the data (like small amplituies in processes dominated by a resonant term).

Nevertheless, analyses like the above-mentioned isobar models go a long way tuward malking such evaluations feasible.

I an going to mention twn of these sum rules explicitly here; both are currently being reported ${ }^{(12)}$ to be in good numerical agreement wi.th experiment.

The Cabibbo-Raricati sum rule ${ }^{(13)}$ ties up the mean-square isovector proton charge radius, $\left\langle r^{2}\right\rangle{ }_{G E}^{V}$, and the total isovector 
nucleon magnetic moment, $\mu^{\mathrm{V}}$ tot, with an integral' over the total absorption cross-section for isovector photons into $I=I / 2$ and $I=3 / 2$ states, $\sigma^{\mathrm{v}}$ tot $(1 / 2)$ and $\sigma_{\text {tot }}^{\mathrm{v}}(3 / 2)$ :

$\frac{1}{6}\left\langle r^{2}\right\rangle_{\mathrm{GE}}^{\mathrm{v}}=\frac{1}{2}\left(\frac{\mu^{\mathrm{v}} \mathrm{tot}}{2 \mathrm{~m}}\right)^{2}+\frac{1}{4 \pi^{2} \alpha} \int_{0}^{\infty} \frac{\mathrm{dk}}{\mathrm{k}}\left[2 \sigma_{t o t} v^{\mathrm{v}}\left(\frac{I}{c^{2}}\right)-\sigma_{\operatorname{tot}}^{\mathrm{v}}\left(\frac{3}{2}\right)\right]$ According to Gilman and Schnitzer ${ }^{(14)}$, the contributions of the various resonances, plus a large non-resonant $S$-wave term resulting from the fits, largely cancel each other under the integral, and the terms other than the integral dominate.

Agreement up to $\mathrm{k}=800 \mathrm{MeV}$ is good to a few percent. The Drell-Hearn sum rule ${ }^{(15)}$ relates the proton anomalous magnetic moment, $\mu_{\mathrm{p}}^{\prime}$, lu an intēgral over total photo-ahsnrption orooo-secliuns for photons with spins paraldel or anti-parullel to the proton spin, $\sigma_{p}$ and $\sigma_{\Lambda}:$

$$
\frac{2 \pi^{2} \alpha}{m^{2}} \mu_{p}{ }^{2}=\int_{0}^{\infty} \frac{d k}{k}\left[u_{p}-u_{\Lambda}\right]
$$

An evaluation by Chau et. al. (16) reports the relation to hold withill the errors inherent in the evaluation of the integral.

\subsection{Other Mesons: $\mathrm{K}$ and $\eta$ Photoproduction in the Isobar Region}

\section{A: $\eta$ Photoproduction}

our interest in the study of the process $\gamma p \rightarrow p \eta$ is due mainly to two features:

1) The isoscalarity of the $\eta$ makes this process a selective 
probe for the $I=I / 2$ channel in $\gamma p \rightarrow p+\left(0^{-}\right)$reactions, whereas the $\pi \mathbb{N}$ final state allows for both $I=1 / 2,3 / 2$.

2) The assignment, together with the $\pi^{\circ}$, of the $\eta$ to the weight -2 position of the $\mathrm{O}^{-}$octet of $\mathrm{SU}(3)$, makes comparisons with the relatively well-studied $\pi^{\mathrm{O}} N$ final state fruitful for tests of symmetry predictions.

Experimentally, the short life-time $\left(\approx 10^{-20} \mathrm{sec}.\right)$ and the many decay modes make $\eta$ production comparatively hard to study. Most photoproduction studies with counter techniques use the partial mode $\eta \rightarrow 2 \gamma$ for definition of the final state ${ }^{(17)}$, although some work has been done using precise momentum analysis of the recoil proton alone. Experiments at CalTech have obtained $\eta$ samples good to $\leq 96 \%{ }^{(18)}$; thereby, not only cross-section data but also information on recoil proton polarization becomes available, and we can hope to look for an analysis of these data in a spirit similar to that outlined for the $\pi \mathbb{N}$ final state in section 1.3 .

The experimental data available at present can be summarlzed briefly as follows: the Bubble Chamber data (19) on the total cross section, shown in Fig. 1.5-1, show a sharp rise above threshold, and a falrly dramatic decrease within $150 \mathrm{MeV}$. Counter experiments Iledsuring differential rross-sections show this feature with somewhat smaller errors. Recent data from Frascati (99) confirm approximate isotropy of the cross-section up to photon energies of $\sim 350 \mathrm{MeV}$ (Fig. 1.5-?). New data from CalTech show up features of the $50^{\circ}, 70^{\circ}$, and $90^{\circ}$ cross sections as shown in Fig. 1.5-3, again confirming the 
$Y p \longrightarrow p \eta$

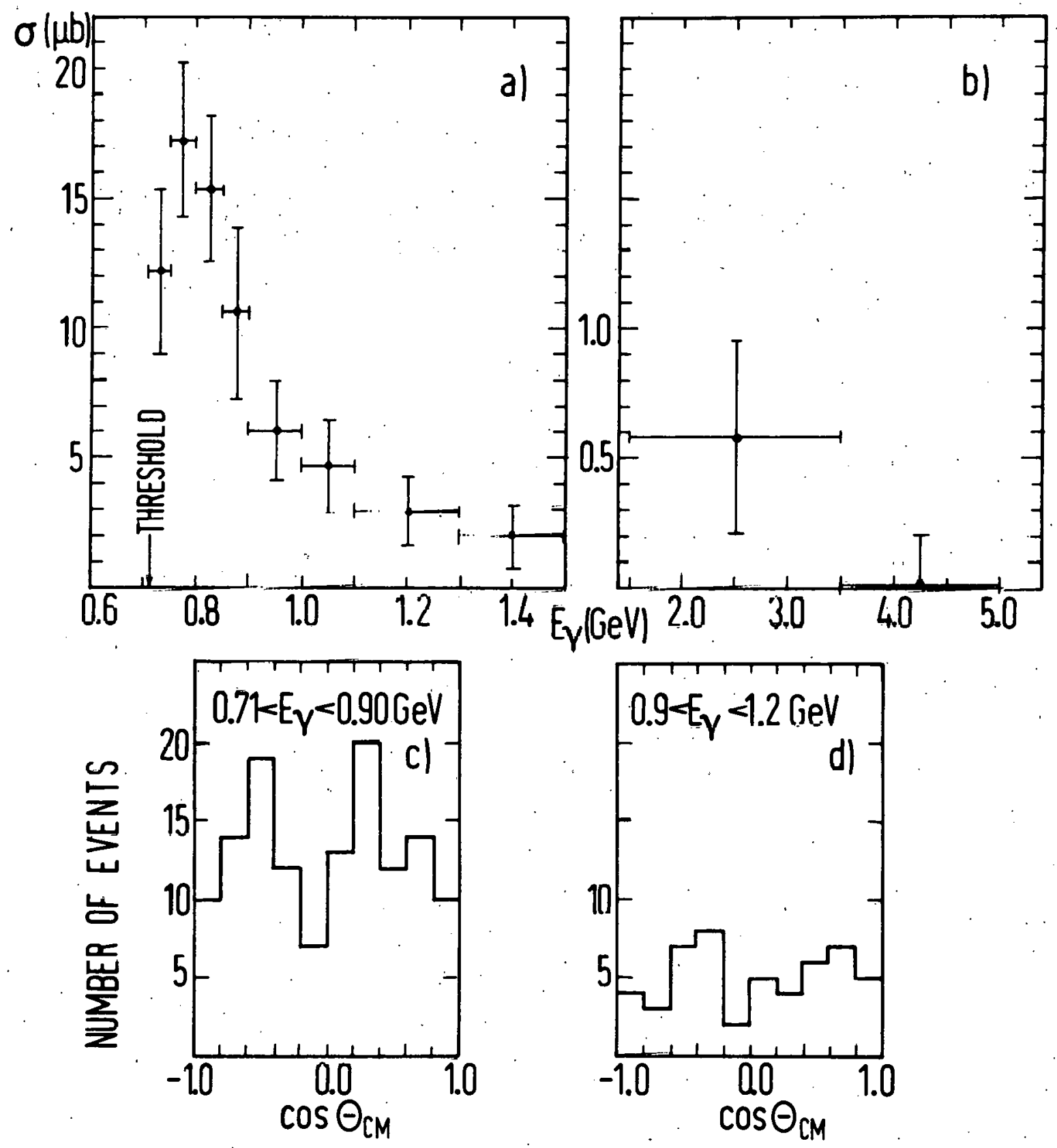

Fig. 1.5-1 Total cross-sections and angular distributions for $\gamma \mathrm{p} \rightarrow \mathrm{p} \eta$, from bubble chamber analysis. (From Ref. No. 19) 


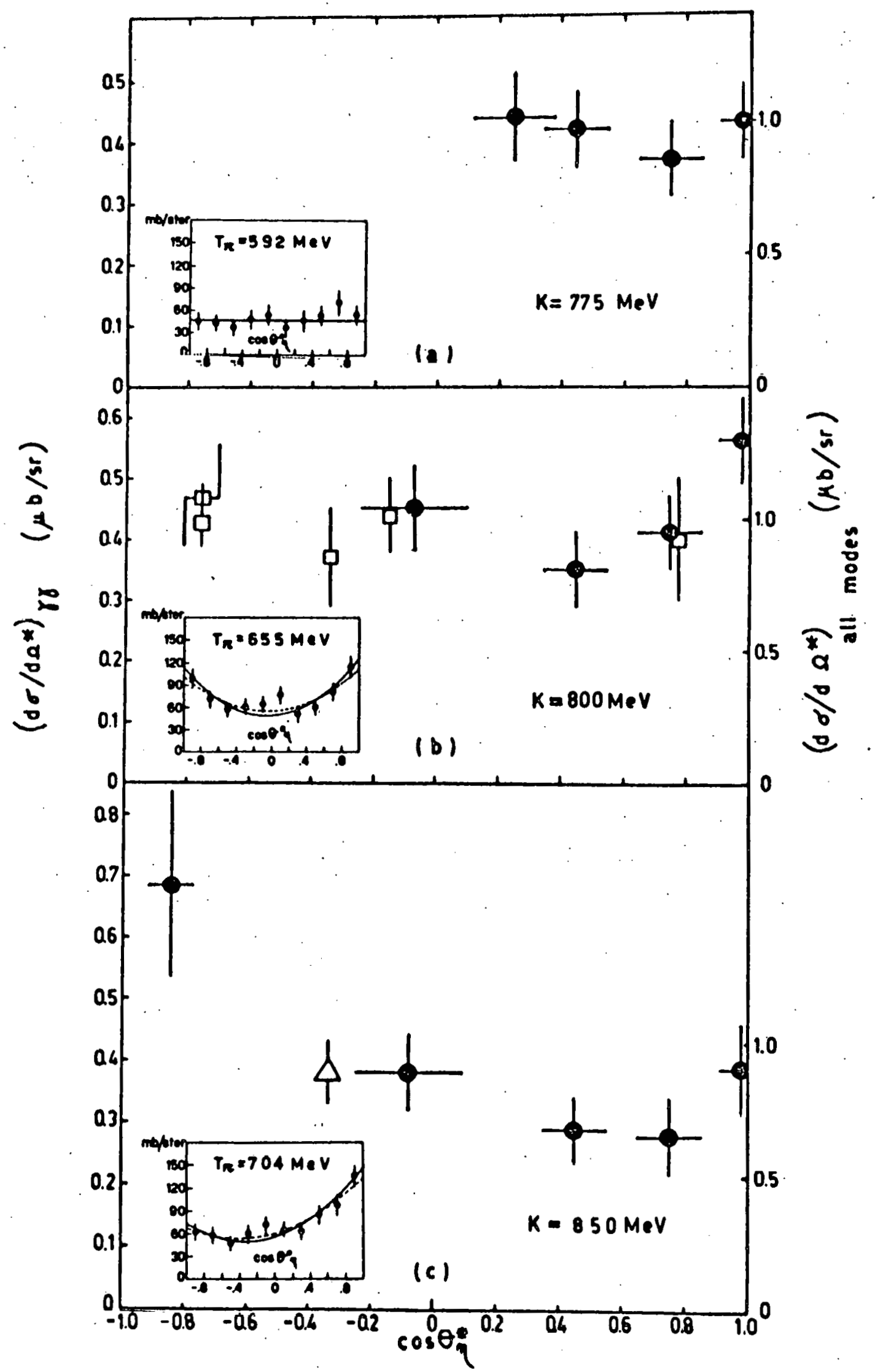

H'ig. 1.5-2 Angular dictributions fnr the differential cross section $\frac{d \sigma}{d \Omega}(\gamma p \rightarrow p \eta)$, from counter experiments at Frascati. Insets show $\pi p$ data at same energies. (From Ref. No. 99) 

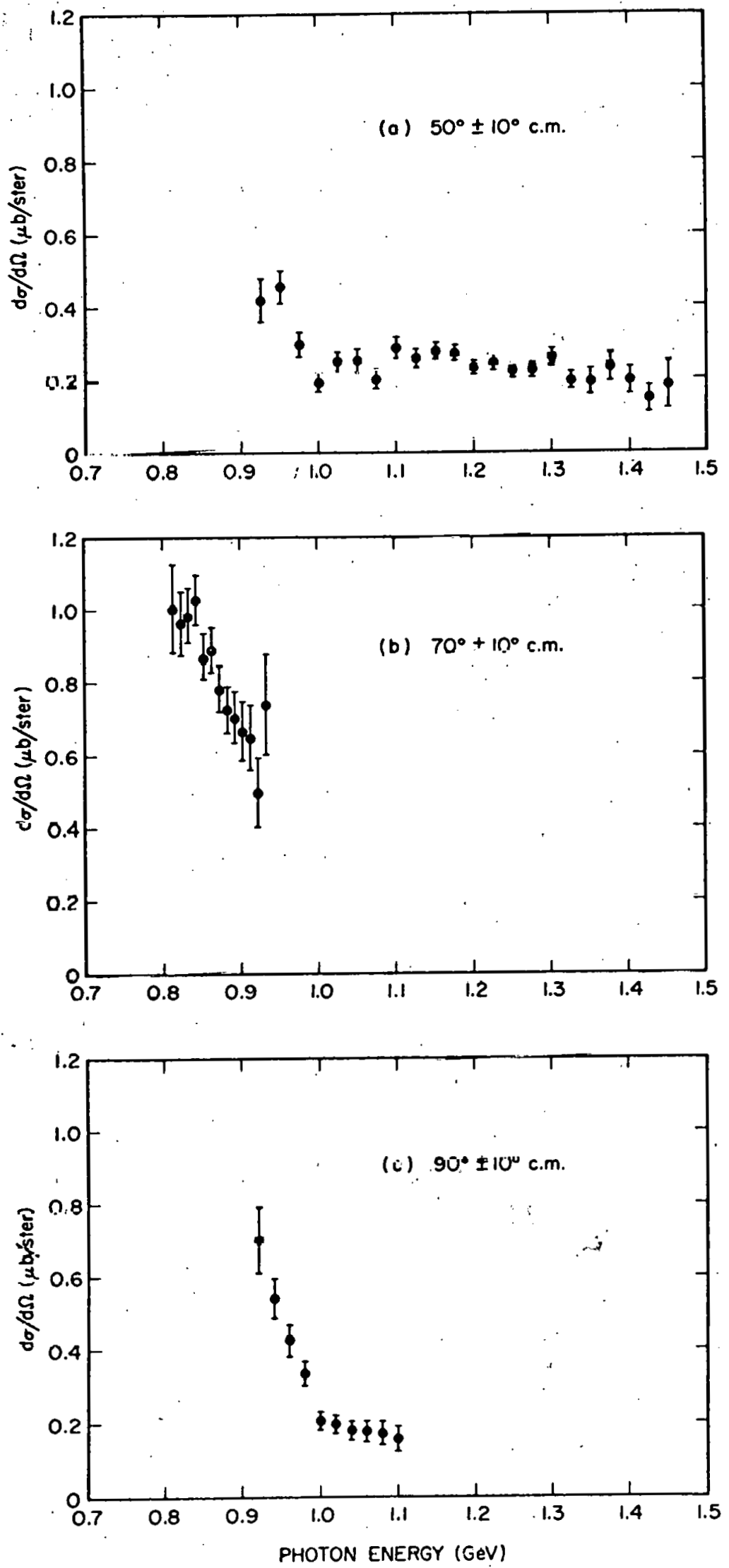

Fig. 1.5-3 $\eta$ photoproduction cross sections at $50^{\circ}, 70^{\circ}, 90^{\circ}$. Caltech counter data.

(From Ref. No. 18) 
quick decay of the $\eta \mathbb{N}$ threshold enhancement, a probable small dip of the $50^{\circ}$ cross-section in the region of $\mathrm{k}=1000-1100 \mathrm{MeV}$, a long shoulder at energies $k=1100-1300$, and a consistently lower value for the $90^{\circ}$ cross-section for $1000 \leq k \leq 1100 \mathrm{MeV}$. Polarization data are not available yet, but have been taken at CalTech, where some 300,000 spark-chamber frames are presently being analyzed.

To try to interpret the cross-section data in terms of some isobar model along the lines discussed in 1.2 , let us look at the possible diagrams. As in section 1.2, we have the Born terms. For s-channel resonances we have the $I=I / 2$ isobars only. We may add vector meson exchange and higher mass poles in the $t$ and $u$ channels.

Fits to the data have been made in this way, involving the s-channel nucleon pole and the $\mathrm{S}_{11}(1570)$ isobar, plus some possible admixtures of $D_{13}(1527), F_{15}$ (1688), and vector exchange ${ }^{(20)}$. With the new data from CalTech extending up to $1450 \mathrm{MeV}$, further work is under way trying to clarify these questions:

1. Can the $\mathrm{S}_{11}$ (1570) alone account tor the lurge enhancement above threshold, or is there some contribution from the $\mathrm{P}_{11}(\sim 1400)$ below thréshöld, or from the $\mathrm{D}_{13}$ (1527)? The latter appears to be ruled out by angular momentum barrier effects $\left(\sim q^{2 b}+1=q^{5}\right.$ near threshold); a contribution of the $\mathrm{P}_{11}$, whose mass is not precisely known, can be isolated only by a $90^{\circ}$ polarization measurement on the recoil proton; it would interfere with the opposite-parity $S_{11}$ and give a large polarization close to threshold. Its presence in photoproduction would pc.se a serious problem to the non-rela- 
tivistic quark model (cf. sections 1.7, 1.8).

2. Since the photoproduction of $\eta^{\prime} s$ through the $F_{15}$ (1688) appears to be strongly suppressed $(27)$, as can be understood in terms of the proper $\mathrm{SU}$ (3) Clebsch-Gordan coefficients ${ }^{(22)}$, we may be able to see whether the shoulder apparent in Fig. $1.5-3$ for the $50^{\circ}$ cross-sertion at 1.1-1.2 GeV is due to decay of the $S_{11}$ (1\%09) isobar, whose presence io hasd lu Uetect in $\pi$ photoproduction (cf, section 1.3). Its presence again poses problems for a quark model interpretation of the photoexcitation of nucleon isobars.

\subsection{B. K Photoproduction}

The photoproduction cross-sections of $\mathrm{K}$ mesons are relatively poorly known when compared with $\pi$ or even $\eta$. $\mathrm{K}^{+}$production, for whilch the most data exist, shows no suggeotive buip structure as we would expect from the previously mentioned date; speciflcally, there is no convincing evidunce for the decay ot the $\mathrm{F}_{15}$ (1688) into $\mathrm{K}^{+} \Lambda$ or $\mathrm{K}^{+} \Sigma$, which we would expect to see (the kinematic threshold excluaes the lowest isobar).

In principle we expect the phennmenology of $K$ prouluction to mun along similar lines to that of $\pi$ and $\eta$ produrtion, with proper repard for the isuspin and strangeness quantum numbers. For charged $K$ production, we expect the diagrams
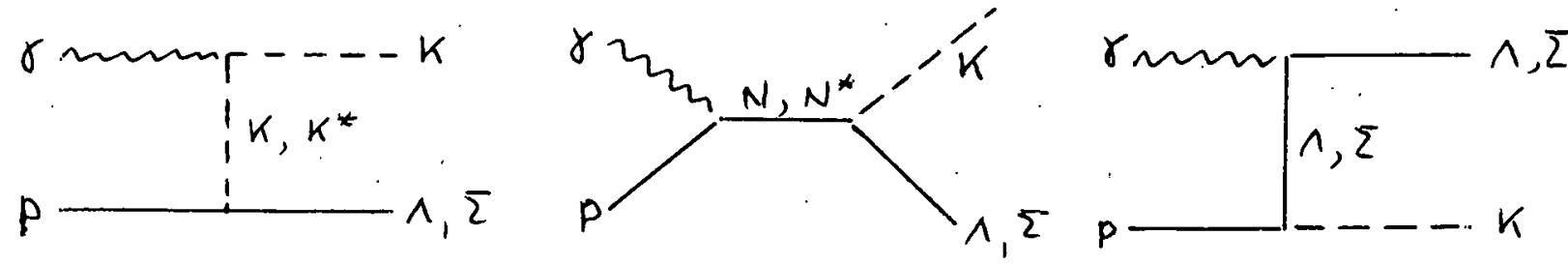
to describe the experimental situation, but the data are too incomplete to allow for a detailed analysis. An additional difficulty is the possibility to produce either a $\mathrm{K}^{+} \Lambda$ or a $\mathrm{K}^{+} \Sigma^{0}$ final state. For charged ' $\mathrm{K}^{\prime} \mathrm{s}$, we can resolve this by looking at the $\mathrm{K}$ yield curve as an excitation function.

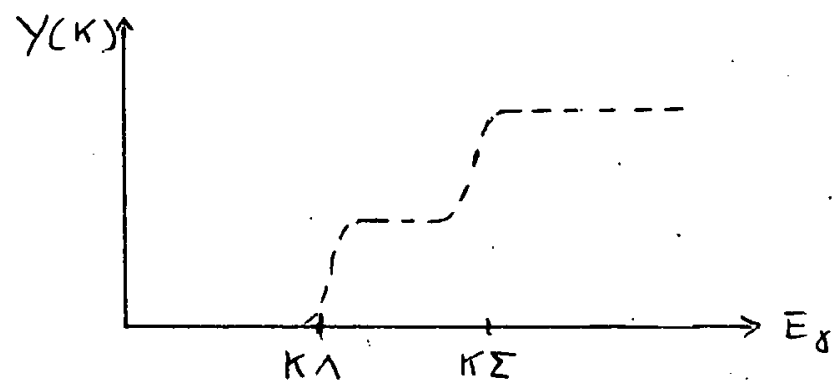

In practice, it is often difficult to separate these final states. A recent experiment by the Yale group at the $\mathrm{CEA}(23)$ has used this excitation function (or "missing mass") technique to investigate the final-state production of strange resonances. Fig. 1.5-4 shows their results as suggestive of the production of various $\mathrm{Y}_{0}^{*}$ 's according to

$$
\gamma \mathrm{p} \rightarrow \mathrm{Y}^{\mathrm{O}^{*}}+\mathrm{K}^{+}
$$

Their results for $Y^{*}$ 's up to masses of $\sim 2600 \mathrm{MeV}$ coincide largely with recent measurements of the $\mathrm{K}^{-}-\mathrm{p}$ total cross-section. (The proper subtraction of multiparticle backgrounds is always a dititicult problem in such experiments).

A similar experiment by the same group switched the polarity of the spectrometer magnets and looked for $\mathrm{K}^{-}$in search of processes like

$$
\gamma \mathrm{p} \rightarrow \mathrm{B}^{++}(\mathrm{S}=+1)+\mathrm{K}^{-}
$$




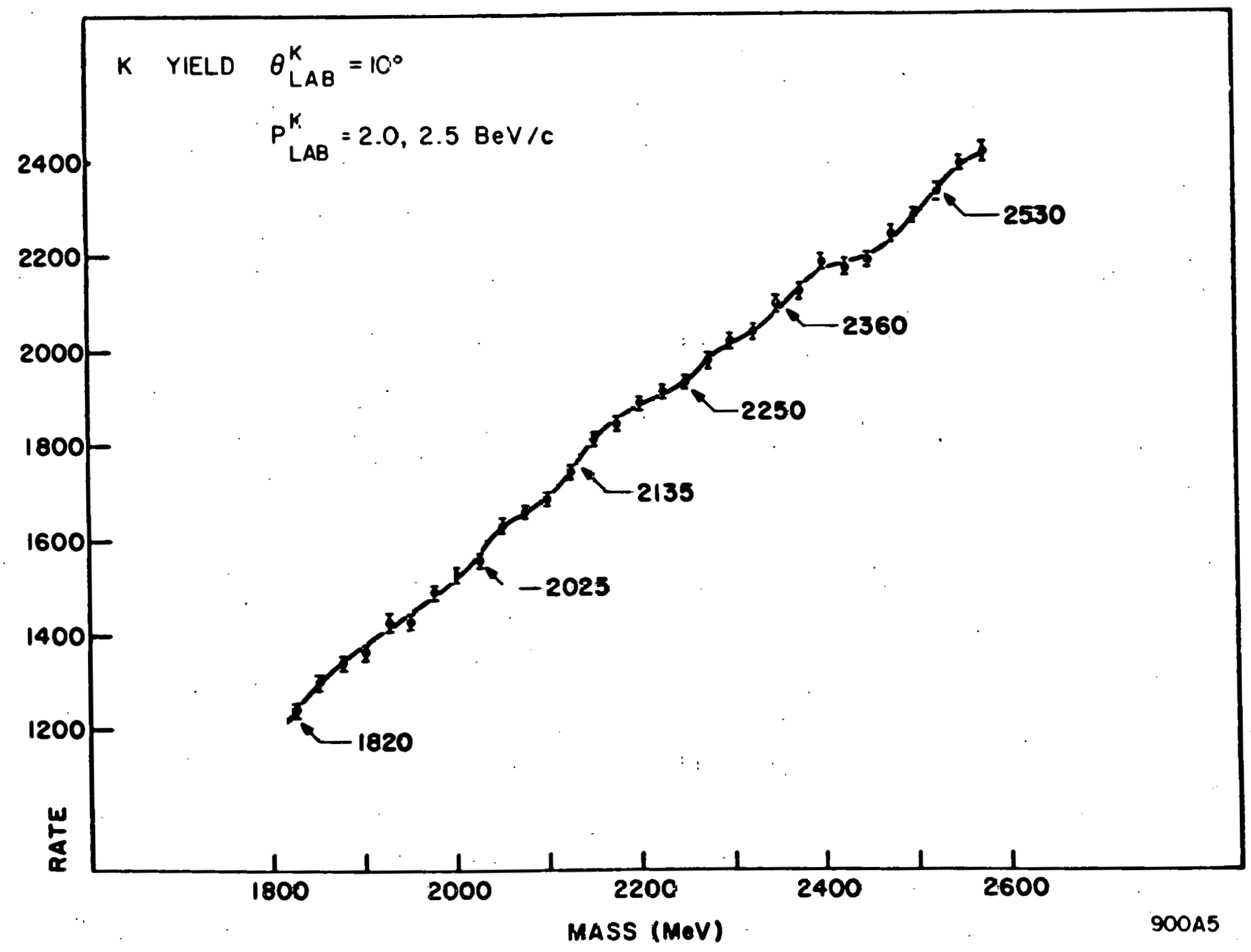

Fig. 1.5-4 $K^{\div}$excitation curve; data is normalized per equivalent quantum. fill data taken at $2.5 \mathrm{Be} / / \mathrm{c}$ and $2.0 \mathrm{BeV} / \mathrm{c}$ spectrometer settings have jeen combined on a missing mass scale. (From Ref. Mo. 23) 


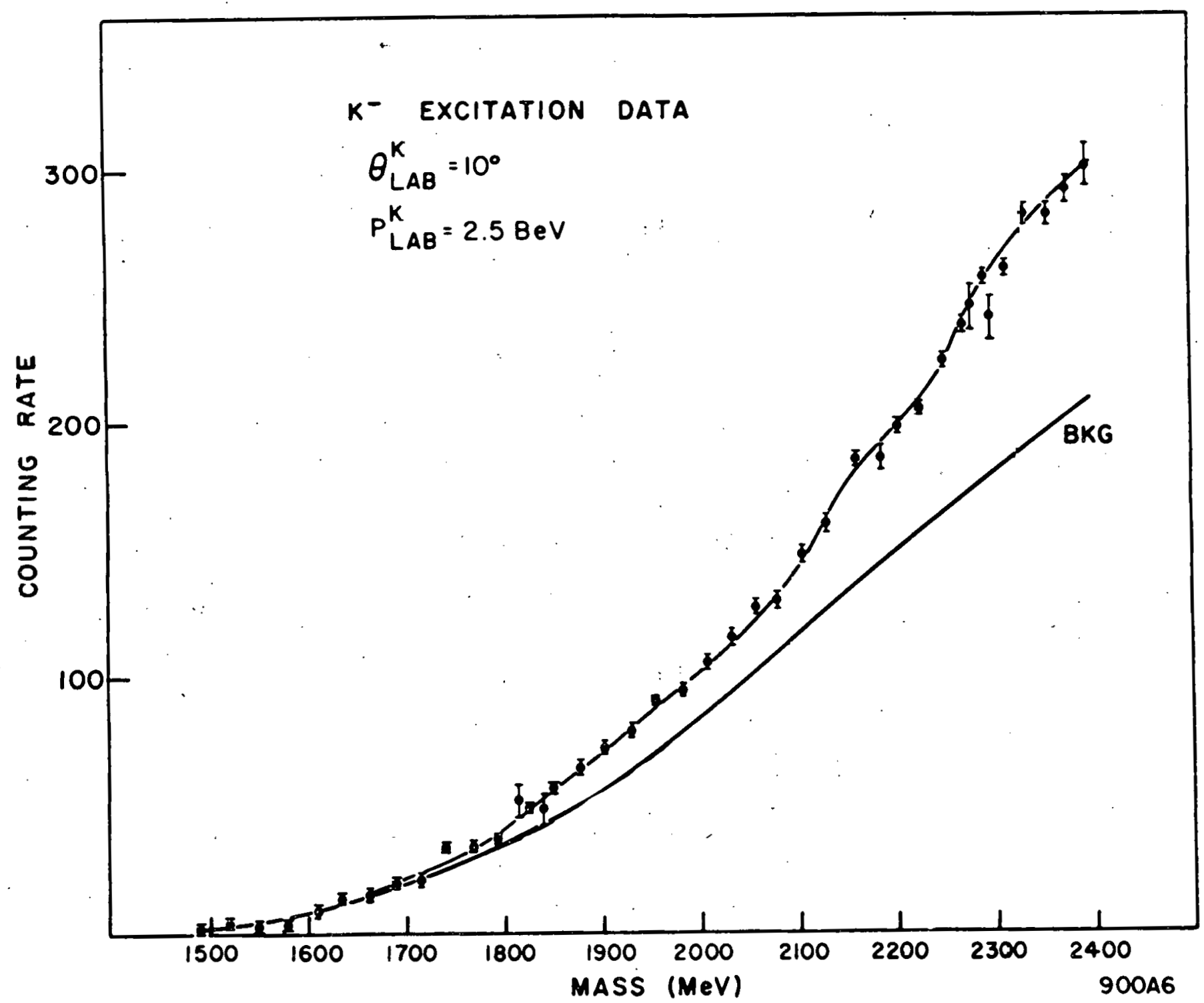

Fig. 1.5-5 $\mathrm{K}^{-}$excitation curve; data is normalized per equivalent quantum. The solid curve shows estimated background from multiparticle reactions. (From Ref. No. 23) 
A comparable structure in the $\mathrm{K}^{-}$yield curve would then indicate the photoproduction of doubly charged strangeness +1 particles. Fig. 1.5-5 may or may not be regarded as evidence for the existence of $S=+1$ baryons: Because of the seriousness of the problem posed to the quark model' by the possible existence of such stateo, mere alrect proof is obviously needed (like $\sigma_{\text {tot }}\left(\mathrm{K}^{+} \mathrm{p}\right.$ ) measurements, which have been giving similar, but inconclusive, indications). Probalily the most extensive use which has hepn made of the proctss $y \mathbb{N}+\mathrm{KB}$ has been the photoproduction of $\mathrm{K}^{\circ}$ beams at high-energy accelerators. This is a very important tonl nf kaon phyoics, sille it provides fairly copious neutral $K$ beams without the curse of heavy neutron, contaminations - which are essentially unavoidable around proton accelerators. The $K^{\circ}$ beam at the Stanford Linear Accelerator is certainly one of the more attractive featureo of that wachine. The observed yields probably come largely from the photoproduction of $\Phi$ mesons which subsequently decay into $\mathrm{K}$ pairs (cf. lecture 2 ).

\subsection{Dispersion Ireatment.}

We have looked at the low-energy data in the photoproduction of pseudoscalar mesons. We saw that simple phenomenological models have d certain amount of success in describing the salient features produced by experiment.

If we want to be more ambitious, we will try and make dynamical models for the photoproduction process. The approach through dispersion relations has had some success at low energies. In the following sections, 1.7 and 1.8 , we will briefly describe some recent 
successes and failures of the unitary symmetry schemes and the quark model, and some suggestions for experiments to be performed.

The dispersion treatment was the first dynamical model which was able to claim some measure of success. The classic paper of CGLN ${ }^{(9)}$ described the region of the $\Delta(1238)$ isobar and has since been considerably refined (see, for an up-to-date review, (24) ). The energy region above $\sim 1400 \mathrm{MeV}$ is hardly open to this approach, since the dispersion treatment hinges on the dominance of the highly elastic $\triangle(1238)$ state.

The basic assumption is that the relevant invariant amplitudes are analytical functions in $s$ and $t$; J. Ball (25) demonstrated that we can then express the full amplitudes in terms of the imaginary part, with no additive terms, by the fixed-t, unsubtracted "dispersion relation"

$$
A_{i}(s, t)=\text { pole terms }+\frac{1}{\pi} \int_{\left(m_{N}-1 \cdot m_{\pi}\right)^{2}}^{\infty} d s^{\prime} \operatorname{Im~} A_{i}\left(s^{\prime}, t\right)
$$

The knowledge of $\operatorname{Im} A_{i}$ then determines the entire reaction amplitude; Im $A_{i}$ is tied up through unitarity with the intermediate states in the $s$ and $u$ channels; a stable state with spin $1 / 2$ and mass $M$ will show up as

$$
\operatorname{Im} A_{i}(s) \sim \delta\left(s-M^{2}\right)
$$

For $J>1 / 2$, additional kinematic factor's will come in, and for a. resonance $(\Gamma>0)$ some Breit-Wigner form will have to be substituted to describe the resonance shape. The important feature is that this treatment not only makes definite statements about the resonant ampli- 
tudes, but the non-resonant amplitudes are also completely defined. In practive, however, calculations are laborious and additional assumptions are needed; for details we have to refer the student to the relevant literature.

\subsection{Isobars and $\mathrm{SU}(3)$}

The conservallun UI isospin in the strong interactions makes precise statements about various decay modes of, let's say, the $\Delta(1238)$. It predicts, e.g., the ratio of $\Delta^{+} \rightarrow \mathrm{p} \pi^{0}$ to $\Delta^{+} \rightarrow \mathrm{n}_{\pi}^{+}$. We can express this in the language of group theory; $p$ and $n, \pi^{\circ}$ and $\pi^{+}$belong to irreducible representations of the $\mathrm{SU}(2)$ group. Transformations within the group take us from $n$ to $p$, from $\pi^{+}$to $\pi^{\circ}$, etc. The practical task is that of finding the proper Clebsch Gordan coefficients which link up the amplitudes.

Bimilarly, higher symmetries can be employed tin make corrusponding predictions. Recall that, by transformation within the $\mathrm{SU}(3)$ group, we can change $\pi$ into $K$ or $\eta, p$.into $\Lambda, \Sigma$, and so on. In the Gidegram

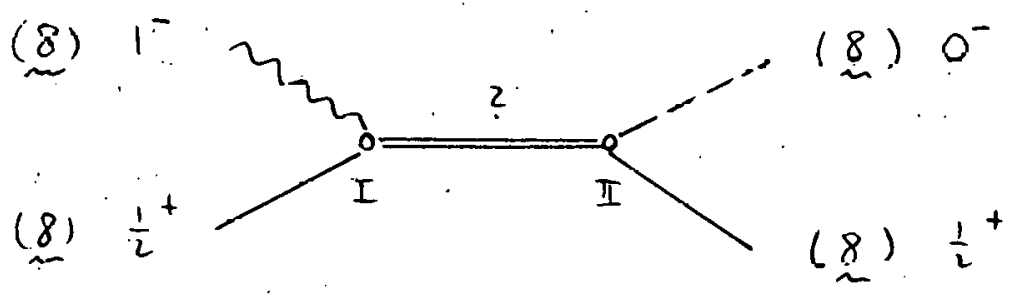

we know the SU(3) assignments for all particles in the initial and final states (assuming that the photon is established as a $U=0$ 
member of an octet). For nucleon isobars, we do not, in general, know the $\mathrm{SU}(3)$ properties. If we want to make statements about vertices I or II, we obviously have to establish the multiplet to which the intermediate state belongs. We know that vertex I will have to conserve $U$ spin, because it is electromagnetic; vertex II is strong, and therefore has to conserve $\mathrm{SU}(3)$.

If we keep these points in mind (and make the proper allowances for kinematical effects), we can write down the $C-G$ coefficients to check against experiment, and maybe decide a posteriori to which irreducible representation the $B^{*}$ in question belongs.

Starting from vertex I, U spin conservation postulates that we photo-excite only $U=1 / 2$ isobars.

$$
\gamma_{\mathrm{p}} \rightarrow \mathrm{N}^{*}\left(\mathrm{U}=\frac{\mathrm{l}}{\hat{c}}\right)
$$

This means that the usual $8 \times 8$ decomposition

$$
8 \times 8 \rightarrow 1+8_{a}+8_{s}+10+\overline{10}+27
$$

has to be scanned for the proper U spin content. This leaves

$$
\begin{aligned}
& \gamma p \rightarrow 8_{a}, 8_{s}, 10,27 \\
& \gamma p \nrightarrow 1, \overline{10}
\end{aligned}
$$

Let's now specialize to the particular case $y p \rightarrow \mathrm{F}_{15}(1688) \rightarrow \eta p$. Verlex II, $\mathrm{F}_{15} \rightarrow \eta p$, has to converve $\mathrm{I}$ spin, which yields (the final

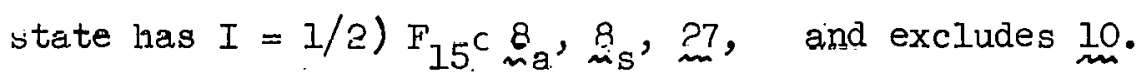
We can now write down the proper CG coefficients for the SU(3) invariant couplinge, and put in appropriate kinematical factors. This gives a prediction for the ratio 


$$
R=\frac{\Gamma\left(F_{15} \rightarrow p_{\eta}\right)}{\Gamma\left(F_{15} \rightarrow p_{\pi}^{\circ}\right)}=\frac{1}{3} \times \begin{cases}3 & \text { if } F_{15} \in \stackrel{27}{m} \\ \frac{1}{3}(3-4 \alpha)^{2} & \text { if } F_{15} \in 8\end{cases}
$$

(where $\alpha$ is a parameter describing the ratio of $\underset{m a}{8}$ to $\underset{m s}{8}$ ).

Experiment then decides that $\mathrm{F}_{15} \in 27$ is excluted, $\mathrm{F}_{15} \subset \stackrel{\mathrm{m}}{\mathrm{m}}$ io indicated with an $\alpha$ parameter of about 0.6 . (22)

We have chosen this example because it shows how the apparcnt absence of the $F_{15}$ isobar in $\eta$ photoproduction. (cf. previous section) can be accounted for in terms of $\mathrm{SU}(3)$ - invariant couplings. Similar arguments can then be used to explain why its decay into $k \Lambda$ also seems to be suppressed. If we assign the $S_{11}(1570)$ to an octet, we can also explain its strong decay into $\eta p$ vs. a weaker $\pi p$ mode, by aupruprlately choosing the $\alpha$ parameter for this mupling; howevcr, eq. 1.7-4 shows that a 27 aseignment may wot be ful. Iy excluded because of the sepiouc decay $\sigma_{11}(1570) \rightarrow \mathbb{N} \eta$.

A special case is the $P_{11}$ (1400), which has so far not been clearly Ldentified in photoproduction. Neither do recent electroproduction data. from the SLAC indicate such a photoexcitation (26) in the proccss ep $\rightarrow e^{\prime} N^{*}$.

This may be die to analyuiu difficulties whlch mask the state's appearance, or to the absence of the vertex $\gamma p \rightarrow \mathrm{P}_{11}$ (1400) altogether (cf. next section). However, there may be some evidence ${ }^{(100)}$ that. while $\gamma \mathrm{p} \rightarrow \mathrm{P}_{11}$ is absent, $\gamma \mathrm{n} \rightarrow \mathrm{P}_{11}$ occurs more freely. If this proves to be true, we will have to assign the $P_{11}$ to a $\overline{10}$ representation of $\mathrm{SU}(3)$, since $\mathrm{U}$ spin conservation tells us that

$$
\gamma p \nrightarrow \overline{10} ; \gamma_{\mathrm{n}} \rightarrow \overline{10} \text {. }
$$


Fuller data on angular distributions and polarization parameters will be needed to decide this question. The simple quark model does not allow for the formation of a low-mass $\overline{10}$, so the question is of importance.

1.8 Isobars in the Non-Relativistic Quark Model

Let us assume that we can group low-mass baryons into three-quark configurations with well-defined wave functions. Dalitz showed (27) that we can assign them to $L=0,1,2$ ( $I=$ total quark angular momentum) states.

The nucleon and $\Delta$ are then $I=O(s)$ states with the same totally anti-symmetric space wave functions, differing only in the spin and isospin parts.

The photoexcitation $y \mathbb{N} \rightarrow \Delta$ can then proceed only through the magnetic dipole, isovector excitation of the nucleon. This checks with our results in section 1.3 .

Now talre $I=I$ ("p stat.es"); we find (27) negative-parity states which form octets ${ }^{2} p_{J}$ (where we identify the non-strange states with the nucleon isobar $S_{11}(1570)$ and $D_{13}(1527)$ ) and ${ }^{4} p_{J}$ (with which we associate the $\mathrm{S}_{11}(1709)$, an unidentified $\mathrm{D}_{13}$, and $\mathrm{D}_{15}$ (1670)). The superscripts 2, 4 indicate total quark spin 1/2, 3/2; the subscript $J$ denotes totel quark angular momentum, i.e., the spin of the baryon. Thus, if we calculate ${ }^{(28)}$ the transition matrix elements for the photoexcitation

$$
\begin{aligned}
& { }^{2} \mathrm{~S}_{I / 2} \rightarrow{ }^{2} p_{\mathrm{J}} \\
& 2_{\mathrm{S}_{1 / 2}} \rightarrow{ }^{4} p_{\mathrm{J}}
\end{aligned}
$$


we find that process $(1.8-1)$ can proceed freely. For (1.8-2), the transition from quark spin $1 / 2$ to $3 / 2$ cannot be effected by the charge or current operator, but only by the magnetic moment operator.

Sandwiching the operator

$$
M=\sum_{i}^{3} \mu_{i} \vec{\sigma}_{i} \cdot(\vec{k} \times \vec{\epsilon}) e^{i \vec{k} \cdot \vec{r}_{i}}
$$

between the appropriate wave functions, Moorhouse ${ }^{(28)}$ showed that the matrix element vanishes for this photoexcitation of protons. For neutrons, no such statement can be made. The prediction is then that

$$
\begin{array}{ll}
\gamma p, n \rightarrow{ }^{2} p_{J} & \text { is allowed } \\
\gamma p \rightarrow{ }^{4} p_{J} & \text { is forbidden } \\
\gamma n \rightarrow{ }^{4} p_{J} & \text { is allowed }
\end{array}
$$

As we showed in sections 1.3 and 1.5 , experimenta.1 evidence indicates that

$$
\begin{aligned}
& \gamma p \rightarrow\left({ }^{2} p_{J}\right) s_{11}(1570), D_{13}(1527) \text { is definitely observed; } \\
& \gamma p \rightarrow\left({ }^{4} p_{J}\right) s_{11}(1709), D_{15}(1670) \text { has not. been extablisher. }
\end{aligned}
$$

The limfts on the latter statement are not reliable, and $\eta$ production may modify our evidence on the photoexcitation of $\mathrm{s}_{11,}$ (1709). We can treat the $I=2$ states similarly, and show that the $\mathrm{F}_{15}$ (1688) may be freely excited in this model. What about the $\mathrm{P}_{11}(1400)$ ? It is usually assigned to $\mathrm{a} \cdot{ }^{2} \mathrm{~s}_{1 / 2}$ configuration with totally symetric space wave-function. (27) In this case, the transition $y N \rightarrow P_{11}$ can be brought about only by a magnetic dipole. But the matrix element corresponding to this 
excitation vanishes because the totally antisymetric nucleon wave function is orthogonal to the $P_{11}$ space part.

Should further experiments in $\pi$ and $\eta$ production confirm the absence of the $\mathrm{P}_{1 I}$, then we can understand it in these terms. Should we find, however, that $\gamma \mathrm{n} \rightarrow \mathrm{P}_{11}$ exists while $\gamma \mathrm{p} \rightarrow \mathrm{P}_{11}$ does not, so that we have to assign this state to a $\overline{I 0}$ in $\mathrm{SU}(3)$, then the framework of this chapter does not apply anyway, because the qqq configuration leads only to $\frac{I}{m}, 8$, and 10 representations. We will close this lecture by remarking that the study of the photoexcitation of nucleon isobars still leaves a number of open questions. Their solution will have an important vearing on our understanding of some aspects of the strong interactions, and on the credibility of various models used to describe them. 


\section{Lecture 2 Photoproduction of Vector Mesons.}

\subsection{Models for the Photoproduction of Vector Mesons.}

We now turn to the photoproduction of mesons with spin-parity quantum numbers $1^{-}$. We will restrict our attention to the pronurtion of vector mesons of hypercharge zero $(S=Y=0), \rho, \omega, \widehat{s}$. Recall from sestion 1.1 thal lhe general production diagram for the neutral $\dot{Y}=0$ vector mesons is distinguished by the fact that no quantum numbers need be exchanged between the top and bottom line of

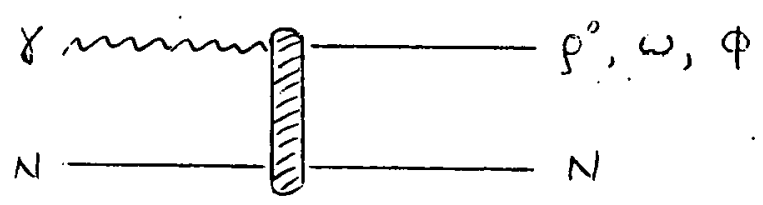

For incoming real photons, with helicity \pm 1 , we have to heed the massiveness of the $1^{-}$mesons ald an additional possibility of spin orientalion (helicity $0, \pm 1$ ). For virtmal photono in lle inltial statc,

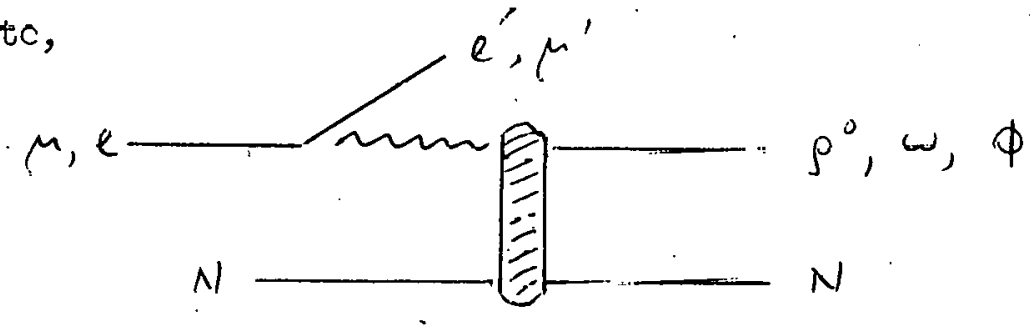

the photon-veclur meson analogy is obviously more complete. $\left(\lambda_{\mathrm{k}}=0, \pm 1\right)$. Recently, a fair amount of experimental material on cross-sections, and some on polarization parameters, has become available. Bcfore we revièw this in sections 2.2-2.4, let us take a brief look at the possible mechanisms for the process $\gamma \mathbb{N} \rightarrow \mathbb{N V}$ :

1. Decay of isobars.

Analogous to the isobar diagrams which dominate low-energy $\pi$ photoproduction, we expect diagrams like 


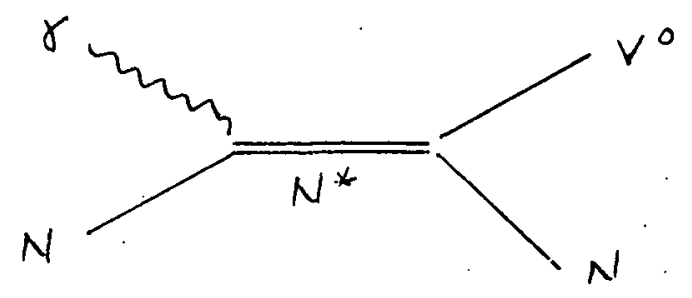

to exist in vector production. However, the lowest-mass isobar whose rest mass will permit the decay $N^{*} \rightarrow \mathbb{N} \rho$ is the $\Delta$ (1920). For isonars of masses $>2 \mathrm{BeV}$, two-body decay modes are probably not dominant. (In section 2.6, we will look at attempts to link up the $\mathrm{N} \rho$ and $\mathrm{N} \gamma$ decays of isobars, connecting the two vertices which make up the mechanism for $\gamma \mathbb{N} \rightarrow \mathbb{N}^{*} \rightarrow N p$ ).

As in $\pi$ production, characteristic angular distributions are expected from the decays of isobars of given J. Moreover, the isospin decomposition of the $\rho N$ system, $\mid I, I_{3}>(\rho, N)$ :

$$
\left.|1,0\rangle\left|\frac{1}{2}, \frac{1}{2}\right\rangle=\sqrt{\frac{2}{3}}\left|\frac{3}{2}, \frac{1}{2}>-\sqrt{\frac{1}{3}}\right| \frac{1}{2}, \frac{1}{2}\right\rangle
$$

makes us look for the decay. of $I=3 / 2$ resonces $\left(\Delta^{\prime} s\right)$ into $p N$, rather than that of $I=1 / 2$ states. $\Delta(1920)$ and $\Delta(2420)$ are obv1ous candidates.

2. One pion exchange (OPE).

The experimentally observed decay $V \rightarrow \gamma \pi$ indicates that the diagram

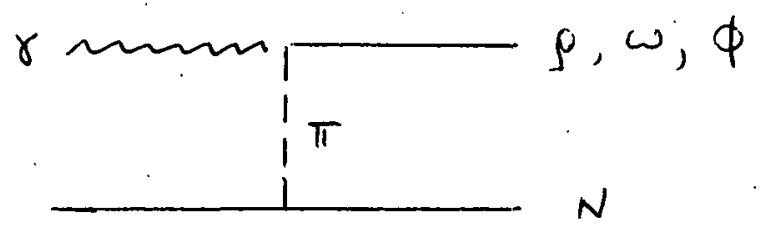


will play a part in vector meson production, and we can link the upper vertex to the radiative decay widths $\Gamma(\rho, \omega, \Phi \rightarrow \pi \gamma)$. If we neglect kinematical corrections due to the mass differences, and in the absence of absorption corrections, we expect a crosssection ratio for the photoproduction of the vector mesons:

$$
\sigma(\rho): \sigma(\omega): \sigma(\Phi)=g_{\rho \mu \eta}^{2}: g_{\omega \pi \gamma}^{2} ; \mathbb{C}^{2} \Phi_{\pi \gamma}
$$

Then the usual assignment of the photon as the $U=0$ member of an SU(3) octet, the conventional mixing of the $I=0$ vector mesons accoraing to

$$
\begin{aligned}
& |\omega\rangle=\cos \theta\left|\omega_{1}\right\rangle-\sin \theta\left|\Phi_{\theta}\right\rangle \\
& |\Phi\rangle=\sin \theta\left|\omega_{1}\right\rangle+\cos \theta\left|\Phi_{3}\right\rangle
\end{aligned}
$$

(with $\mid \omega_{1}$ v, $\mid \Phi_{R}>$ the pure singlet, and octct $I=0$ states), plus the assumption that the $V \pi \gamma$ vartices conserve SU(3), y1etr a sum rule for the coupling conntanti:

$$
\sqrt{3} \dot{g}_{\rho \pi \gamma}=\sqrt{\frac{2}{3}} g_{\Phi_{\pi \gamma}}+\sqrt{\frac{1}{3}} g_{\omega \pi \gamma}
$$

Here, we have taken mixing angles from the Gell-Maru-Okubo mass formula, $\cos ^{2} \theta=\frac{2}{3}$.

Experimentally, the deoay $\rightarrow \pi y$ has not been observed. Deviral arguments have been presented to explain this feature; the most straight-forward comes from the quark model: if the electromagnetic transition proceeds via emission ot' a photon by one quark, then the quark structure of the $\Phi$ (which consists of two strange quarks, $\lambda \bar{\lambda}$ ) forbids the vertex $\Phi \pi \gamma$ (two non-strange quarks make 
up the $\pi$ ).

This leaves us with the distinctive OPE prediction that $g^{2} \omega_{\pi \gamma}=9 g^{2}$. $\frac{1 \gamma}{\gamma}$ and therefore

$$
\sigma(\omega)_{O P E}=9 \sigma(\rho)_{O P E}
$$

We also expect, from $g^{2} \Phi_{\pi \gamma} \approx 0$, that $\sigma(\Phi)_{O P E} \ll \sigma(\rho, \omega)_{O P E}$.

3. Diffraction mechanism.

We note that the diagram

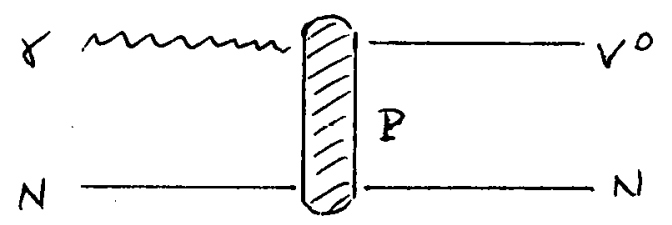

may proceed through the exchange of a system $P$ with the quantum numbers of the vacuum. This can then be viewed in strict analogy with highenergy elastic scattering of $\pi^{\prime} s$ or $K^{\prime} s$ off protons. We therefore expect a diffraction-like picture to ensue at high energies. The distinctive features of diffractive processes like these are then the folluwing, lnow from high-energy $\pi^{+} p \rightarrow \pi \pm p$.

$$
\text { I) } \frac{d \sigma}{d t} \approx a e^{b t} \quad \text { where }\left\{\begin{array}{l}
\left.\frac{d \sigma}{d t}\right|_{t=0,}=a \\
b \text { is of order } 8-10(\mathrm{GeV} / \mathrm{c})^{-2}
\end{array}\right.
$$

II) ${ }^{\sigma}$ tol $\rightarrow$ const. a.t high energies,

III) The forward amplitude is purely imaginary. (cf. eq. 2.1-7) We alan expect diffractive photoproduction to proceed coherently on nuclei, with a corresponding dependence of the parameter a 
on the atomic number $A$ of order $a \sim A^{>1}$.

The characteristic diffraction peak can be seen as resulting from a coherence condition ${ }^{(29)}$ at high energies: the process most likely to occur in high-energy $\gamma \mathrm{N}$ collisions is inelastic meson production. Elastic scattering, whenever appreciably large; is eoocntially shadow scattering due to the existence of these inelastic channels. It an therefore be approximately described in terms of sums over inelastic processes (running either way). In fact, the forward peak is then due to the coherence criterion that cach inelastic channel, $f_{a n}$, returm to the initial state, $a$, via the amplitude $f_{n a}$; this yields for the "shadow" amplitude:

$$
\begin{aligned}
\operatorname{Im} f_{a a} & =\sum_{n} f_{a n} f_{n a}^{*} \delta^{4}\left(p_{n}-p_{a}\right) \\
& =\sum_{n}\left(f_{a n}\right) * \delta^{1}\left(p_{n}-p_{a}\right)
\end{aligned}
$$

In terms of a Regge pole analysis, we look at the exchanged object in the diffraction diagram as the vacuum trajectory $P$. This, the Pomeranchuk trajectory, has, for forward scattering, the angular momentum $\alpha(t=0)=1$. We therefore tind for the amplitude

$$
\begin{aligned}
& A(t) \sim \text { is } \alpha(t) \\
& A(t \rightarrow 0) \sim \text { is }(\alpha(t \rightarrow 0) \approx 1)
\end{aligned}
$$

Then, if the trajectory $\alpha_{p}(t)$ has a positive slope, we expect for the physical region, $t<0$,

$$
\alpha(t<0) / \alpha(0)<1
$$


This leads to the well-known shrinkage of the diffraction peak. Failure of the data to produce evidence for this shrinkage has to be taken as evidence that either the $P$ trajectory has a small slope. (or zero slope: fixed pole at $J=1$ ); or that other trajectories contribute in adaltion.

In the context, of $\mathrm{SU}(3), \mathrm{P}$ is a unitary singlet. Then with the assumptions on the symmetry properties of photon and $\mathrm{V}^{\mathrm{O}}{ }_{\mathrm{s}}$ as above, SU(3) predicts the cross-section relation

$$
\begin{aligned}
\sigma(\rho)_{\text {diff. }}: \sigma(\omega)_{\text {diff. }}: \sigma(\Phi)_{\text {diff. }} & =3: \sin ^{2} \theta: \cos ^{2} \theta \\
& =9: 1: 2
\end{aligned}
$$

Notice that the prediction is diametrically opposed to the OPE picture, where $\omega$ production is much more abundant than $\rho^{0}$ production. For a clean test of the OPE picture, we would have to resort to a process which cannot also proceed diffractively, like $\gamma \mathrm{p} \rightarrow \mathrm{V}^{\mathrm{O}} \Delta^{+}$, where we then expect

$$
\left.\frac{\sigma\left(\gamma p \rightarrow \Delta^{+} \omega\right)}{\sigma\left(\gamma p \rightarrow \Delta^{+} \rho^{\circ}\right)}\right|_{\mathrm{OPE}} \approx 9
$$

For the total production cross-section, due to the OPE graph, we expect an energy dependence of urder $\mathrm{k}^{-2}$ (.cf. ref. 4?),

The OPE and diffractlon pictures leave us with some specific, and contradictory, predictions on production rates. Let us keep in mind that we had to make certain assumptions in order to arrive at these predictions.

The next three sections will present some experimental data, and in section 2.5 we will attempt to arrive at a unif'ied picture. 
2.2 $\rho$ Photoproduction.

The isovector $\rho$ meson $\left(J^{P G}=I^{-+}, C=-I\right)$ has been widely studied, in photoproduction, in its neutral charge state. This is due to its ample production rate and to its simple and distinctive final state $\pi^{+} \pi^{-}$. The other charge states $\left(\rho^{+} \rightarrow \pi^{+} \pi^{\circ}\right)$ have a neutral in the rinal state and are therefore harder to observe. Few data exisl; and since our Interest lieo largely in the analogy photon (neutral) vector meson, we will restrict our treatment here to the $I_{3}=0$ state.

Although the $\pi^{-1} \pi^{-}$final state is easy to detect both in bubble chamber and in counter experiments, the presence of other mechanisms that can produce these, makes an unambiguous determination of mass and width of the $\rho^{\circ}$ difficult. Fig. 2.P-7. show the tutel uross 3cetiun, at energies up to $\sim 5.5 \mathrm{GeV}$, for the proccis

$$
\eta p \rightarrow p \pi^{+} \pi^{-}
$$

where the dominant contributions come from

$$
\begin{aligned}
& \left.\gamma p \rightarrow \begin{array}{c}
\wedge^{++} \pi^{-} \\
\Delta^{0} \pi^{+}
\end{array}\right\} \rightarrow p \pi^{+} \pi^{-}, \\
& \gamma \mathrm{p} \rightarrow \mathrm{p} \rho^{\circ} \rightarrow \mathrm{p} \pi^{+} \pi^{-} \text {. }
\end{aligned}
$$

In order to disentangle the total $\mathrm{p} \pi^{+} \pi^{-}$yield, a Dalitz plot of invariant masses $\mathrm{m}^{2}\left(\mathrm{p} \pi^{+}\right)$vs. $\mathrm{m}^{2}\left(\pi^{+} \pi^{-}\right)$can be made to show that, at low energies, there are three terms to be taken into account, i:e. processes 2.2-2, 2.2-3, and a non-resonant background. Putting in 


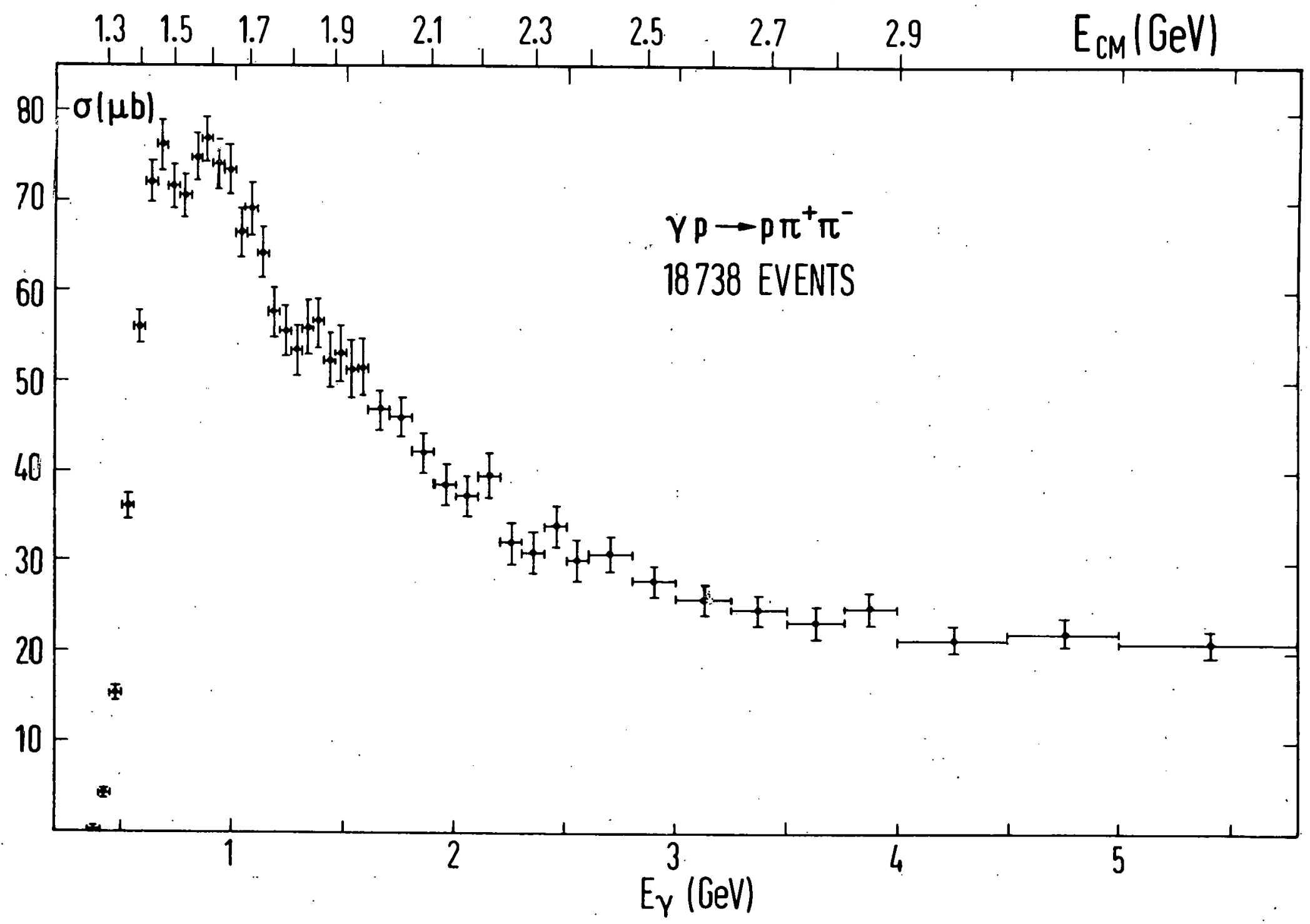

Fig. 2.2-1 Total cross-section for $\pi^{+} \pi^{-}$photoproduction on protons. (From Ref. No. 19) 
appropriate expressions for the resonance contributions, the $\Delta^{++}$ term can be separated out. The remaining problem is the discrimination of $\rho$ production from the uncorrelated background. Fig. 2.2-2 shows the $\rho$ yield as a function of photon energy, using three different fitting procedures. The two mass distribution functions that gave good fits to the bubble chamber data at intermediate energies ( $1.5 \leq k \leq 5.5 \mathrm{GeV}$ ) are characterized by

a) A Breit-Wigner distribution multiplied by a massskewing factor $\left(\frac{m}{m_{\pi \pi}}\right)^{4}$ from the diffraction dissociation model of Ross and Stodoliky ${ }^{(30)}$; it olught to be stressed that this factor is not a necessary ingredient of the diffraction picture.

b). A Breit-Wigner distribution with energy-dependent width interfering with a background of slowly varying phace ${ }^{(31)}$. Thils model also assumes a. purely imaginury forward amplitude for $\rho^{\circ}$ production, as in the diffraction mechanism.

Both of these fits tend to lower the resulting mass valuc for the $\rho$. It is important to keen in mind thel the mass and width parameters for the $\rho$ arc strungly dependent on the fitting process chosen, and therefore differ apprccialily vetween various experimentis. Thu most reliable experimental results on $m_{\rho}, \Gamma_{\rho}$ were recently quoted by Auslander et al., (32) who used $\mathrm{e}^{+} \mathrm{e}^{-}$collisions to study the process

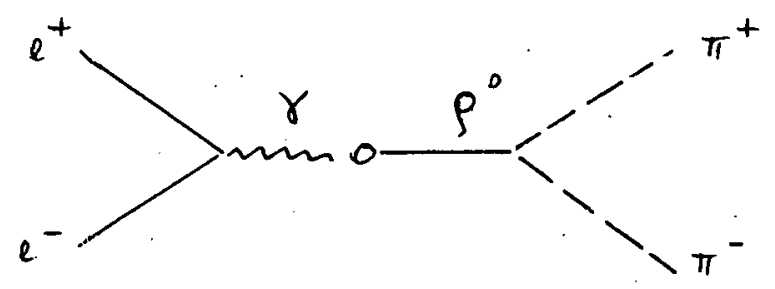




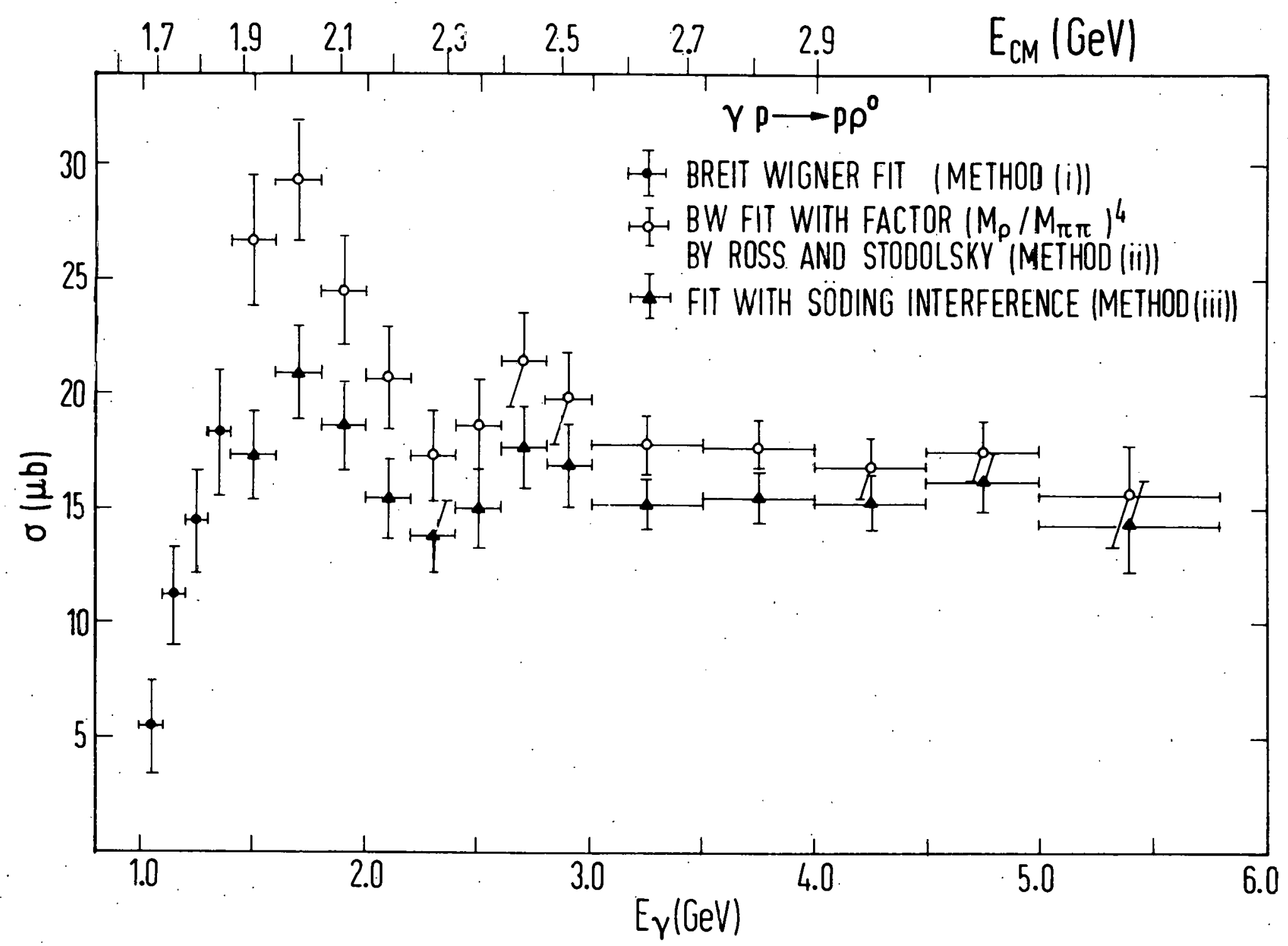

Fig. 2.2-2 Total crosi-section for the process $\gamma p \rightarrow$ p $\rho^{\circ}$. Values vary according to fit employed for separation of these data from those s.jow in Fig. 2.2-1. Bubble chamber data. (From Ref. No. 19) 
and found (cf. Fig. 2.2-3) $\mathrm{m}_{\rho}=764111 \mathrm{MeV}, \Gamma_{\rho}=93 \pm 15 \mathrm{MeV}$. This compares with photoproduction results of $\mathrm{m}_{\rho} \approx 770 \mathrm{MeV}, \Gamma_{\rho} \approx 150 \mathrm{MeV}$. There is at present no full understanding for the difference in these parameters as resulting from $\mathrm{e}^{+} \mathrm{e}^{-}$annihilation and photon-hadron or hadron-hadron collisions. (33) However, it is certainly not surprising that the width parameter of a particle with the lifetime of the $\rho$ should be sensitive to the d.jfference belween strong and electromagnetic formation processes:

The results of the bubble chamber data at DESY and CEA can be summarized as follows:

I. At lower energies, $\mathrm{k} \leq 2.8 \mathrm{GeV}$, there is some structure in the cross-section. The differential cross-section $\frac{d \sigma}{d t}$ shows some diffraction-type behavior (Fig. 2. $\hat{\varepsilon}-4$ ) pIus more isotropic contributions, presumahly from isular decay. (cI. section 2.1 ): We can take ulut events in the forward, diffraction-peak, region, and look at the remaining events. Fig. 2.2-5 shows a suggestive peak at. the locatiun of the $\Delta(2420)$ isobar, and an chlnaument below $2 \mathrm{GeV}$ which may be die to $\triangle(1 \ni 20)$ decay and/or an OPE contribution.

2. At high energies, the alffraction picture appears to describe the experiment well. The forward cross-section can easily be fitted by an expression of the type

$$
\begin{aligned}
\frac{d \sigma}{d t}\left(\gamma p \rightarrow p_{\rho}^{\circ}\right)=a e^{b t} \\
\text { with }\left\{\begin{array}{l}
a \approx 140 \mu \mathrm{b} / \mathrm{GeV}^{2} \\
b \approx 8 \mathrm{GeV}^{-2}
\end{array}\right\}
\end{aligned}
$$




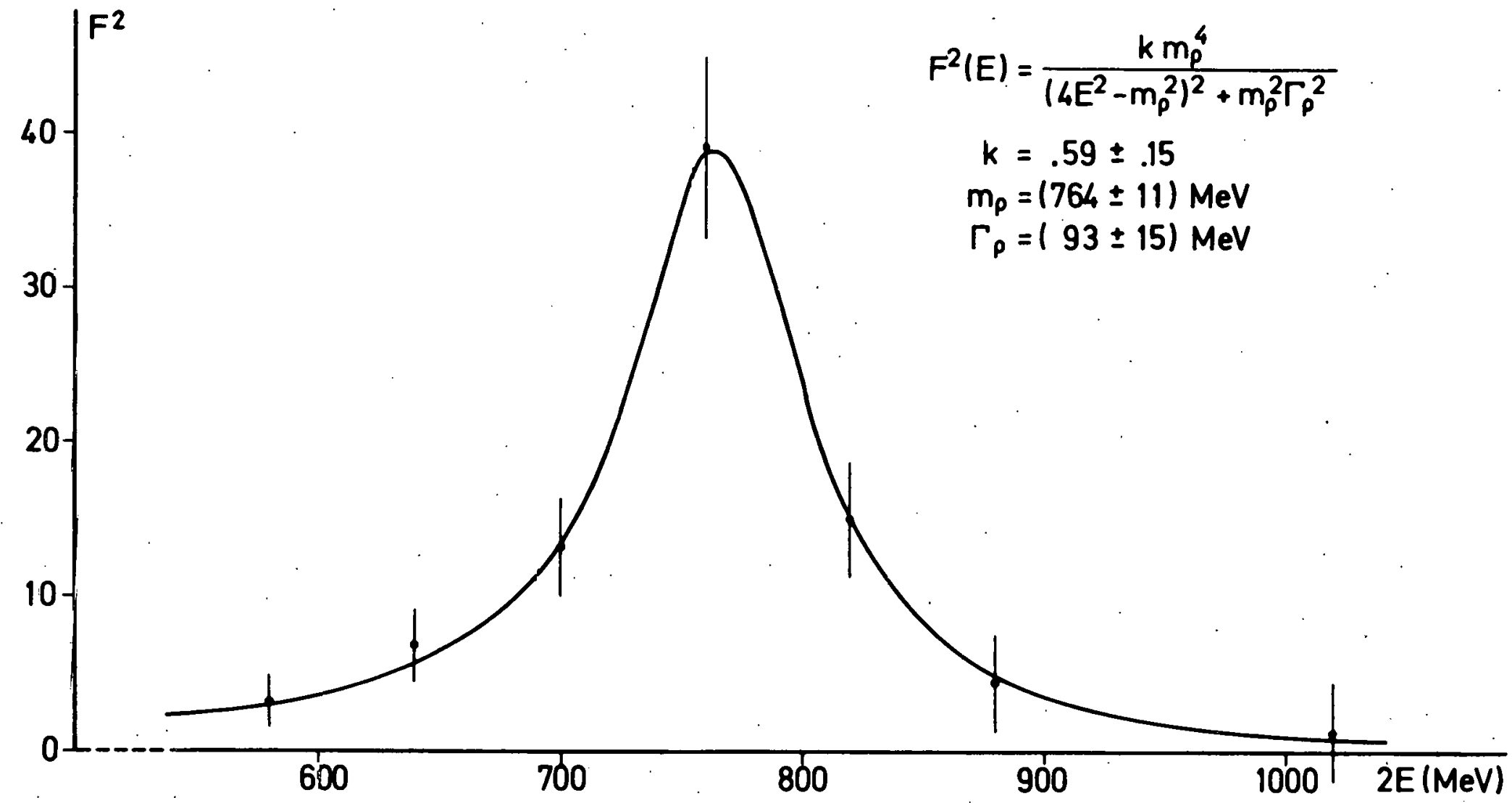

Experimental values of $F^{2}(E)$ approximated by the Breit-Wigner formula

Fig. 2.2-3 $\rho$ resonance shape as observed in first storage ring experiment $e^{+} e^{-} \rightarrow \rho^{0}$, in Novosibirsix. (From Ref. No. 32) 

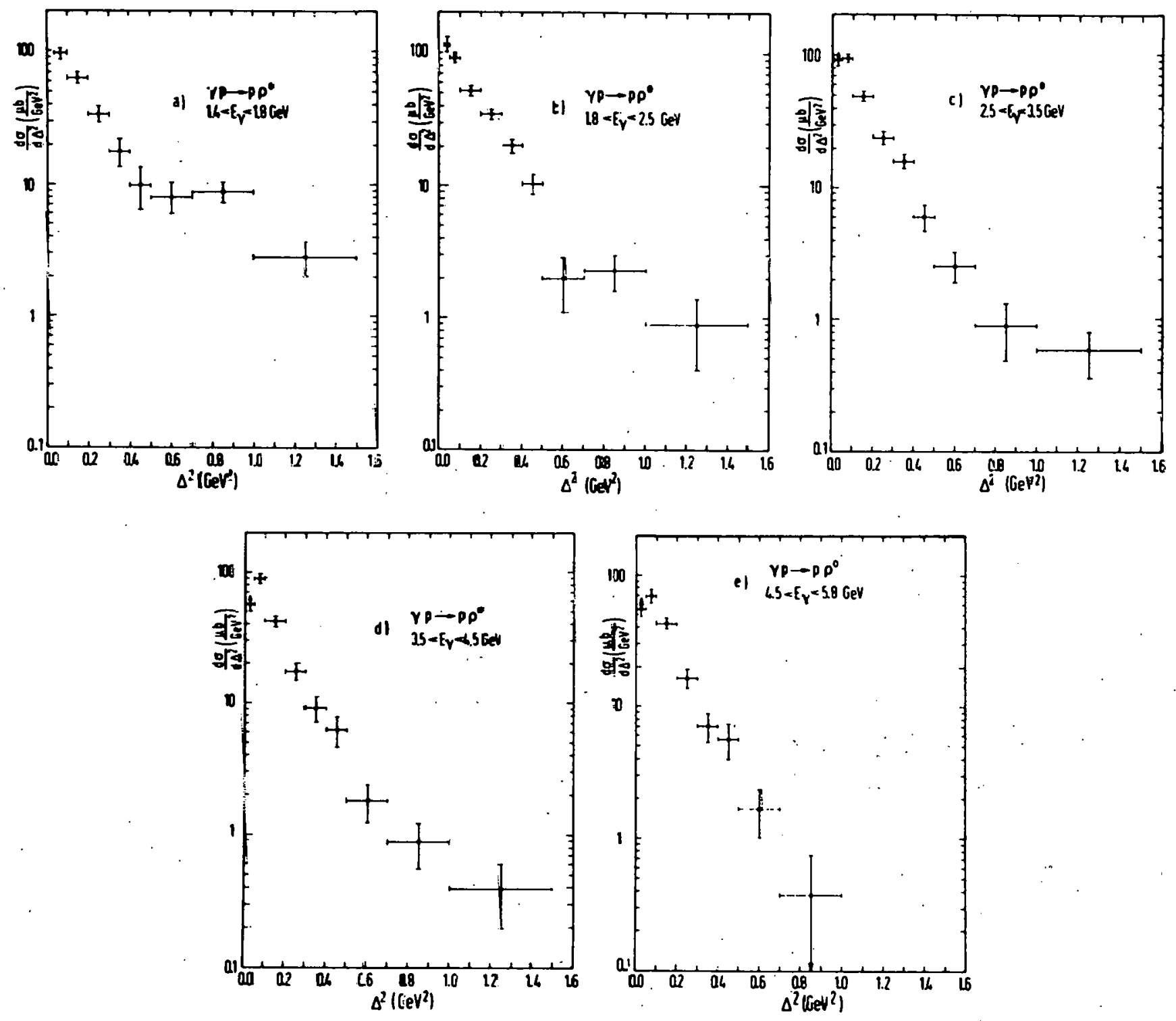

Fig. 2.2-4 Diffङrential cross-sections $\frac{d \sigma}{d S_{i}}$ for $f$ photoproduction. Bubble chamber da (From Ref. No. 19) 


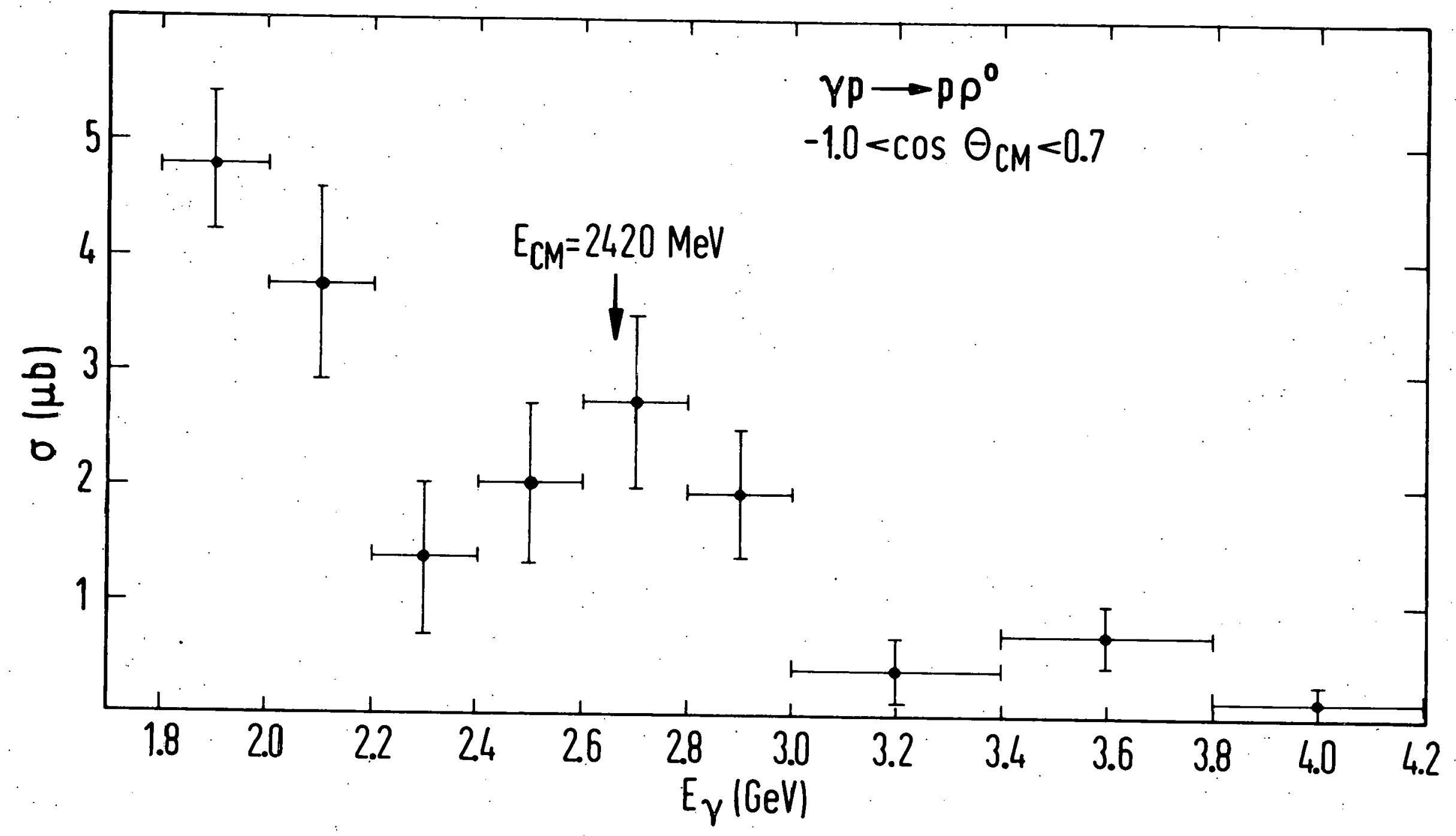

Fig. 2.2-5 Total cross-section for $\gamma p \rightarrow p \rho^{\circ}$, excluding forward (diffraction) production. (From Ref. No. 19) 
For precise numbers, cf. Table I. The total cross-section remains roughly constant at $15 \mu \mathrm{b}$ between 3 and $6 \mathrm{GeV}$.

3. The decay angular distributions are (conveniently expressed in terms of the helicity density-matrix elements $\rho_{\text {ik }}$ of the $\rho^{\prime}$ s) such that one cannot draw any strong conclusions as to the modcls prupused. In particular, predictions of the "strong absurption model" usen hy the OLA gruup (34), incorporating diffraction scattering off a spin-independent potential, seem to be at best partially met (for details we have to refer to the relevant literature $(34,19,35)$ ).

There are several recent counter experiments reporting $\rho^{\circ}$ photoproduction results to complement this picture. Since they employed very different techniques to detect the $p \pi^{+} \pi^{-}$final state, let's have a short look at them.

1. 1nsest in teclululue to the bulule chamber experiment in Lhe observation of threc-charged-prong events in a streamer chamber, recently reported from SLAC by Davier et. al. (36). A thin hydrogenfilled reaction tube inside a large streomer chamber allowed the determination of the killumatics fairly completely. This is a new technique, distinguisher from the unbs le chamber approach hy the puodibillty of triggering the detection system selectively. Although, in this first experiment with such an instrument, there still remain some problemc and pussible biases, several interesting features result from the analysis of $\approx 850 \mathrm{p}^{+} \pi^{-}$events, at photon energies from 6.5 to $17.8 \mathrm{GeV}$ : 
Takle I. Data on Vector Mesons - Photoproduction and Decay Widths

\begin{tabular}{|c|c|c|c|c|c|}
\hline Parameters & (units) & $\rho^{0}$ & $\omega$ & $\Phi$ & Experiment at $\mathrm{E}_{y}(\mathrm{GeV})$ \\
\hline . & $\begin{array}{l}m(\mathrm{MeV}) \\
\Gamma(\mathrm{MeV})\end{array}$ & $\begin{array}{l}764 \pm 11 \\
100 \pm 12 \\
130 \text { to } 150\end{array}$ & $\begin{array}{r}783 \pm 0.7 \\
14.0 \pm 2.4 \\
12.2 \pm 1.3\end{array}$ & $\begin{array}{r}1019 \pm 0.6 \\
4.2 \pm 0.9 \\
3.4 \pm 0.8 \\
\end{array}$ & $\begin{array}{l}\text { (Rosenfeld tables) } \\
\mathrm{e}^{+} \mathrm{e}^{-} \rightarrow \mathrm{V}^{\circ} \text { (Orsay) } \\
\text { hadronic interactions }\end{array}$ \\
\hline \multirow{2}{*}{$\frac{d \sigma}{d t}\left(r p \rightarrow p V^{o}\right)=a e^{b t}$} & $\mathrm{a}\left(\mu \mathrm{b} \mathrm{GeV}^{-2}\right)$ & $\begin{array}{r}1 \leq 0 \pm 12 \\
125 \pm 15 \\
140 \pm 50 \\
\approx 30 \pm 30 \\
\end{array}$ & $28.4 \pm 5.2$ & $1.6 \pm 0.6$ & $\begin{array}{l}\text { J bubble chamber }<6 \\
\text { counter, spark chamber }<6 \\
\text { spectrometer }\end{array}$ \\
\hline & $\mathrm{b}\left(\mathrm{GeV}^{-2}\right)$ & $\begin{array}{l}8 \pm 0.7 \\
8.1 \pm 1.5 \\
\approx 10 \\
\approx 8.5\end{array}$ & $7.6 \pm 1.2$ & $3.5 \pm 0.9$ & $\begin{array}{l}\text { bubble chamber }<6 \\
\text { counter, spark chamber } \\
\{\text { spectrometer } 6-18\end{array}$ \\
\hline$\sigma_{\text {tot }}\left(V^{O} p \rightarrow a l l\right)$ & $(\mathrm{mb})$ & $\begin{array}{c}31.7 \pm 2.3 \\
-28\end{array}$ & 28 & $\begin{array}{l}13.4 \pm \\
9.7 \pm 2.0 \\
7.2 \pm \\
11.5 \pm 1.5\end{array}$ & $\begin{array}{cc}\text { from bubble chamber } & <6 \\
\text { and } & \\
\text { from spectrometer data } & 13 \\
\text { frcm guark model, } \pi N, \text { KN data } & \end{array}$ \\
\hline$\Gamma\left(v^{0} \rightarrow e^{+} e^{-}\right)$ & $(\mathrm{keV})$ & $\begin{array}{l}6.4 \pm 0.72 \\
\text { ( similar } \\
\text { values from } \\
\text { many other } \\
\text { experiments })\end{array}$ & $\begin{array}{l}1.1 \pm 0.25 \\
0.49 \pm 0.19\end{array}$ & $\begin{array}{l}1.3 \pm 0.21 \\
1.1 \pm 0.33 \\
2.1 \pm 0.9\end{array}$ & $\begin{array}{l}\text { Orsay } e^{+} e^{-} \rightarrow V^{\circ} \\
\text { DESY } V^{\circ} \rightarrow e^{+} e^{-} \\
\text {CERN } V^{\circ} \rightarrow e^{+} e^{-}\end{array}$ \\
\hline
\end{tabular}


To fit the $\pi^{+} \pi^{-}$mass distribution well, a $\rho$ Breit-Wigner form has to be multiplied by the mass-skewing factor $\left(\frac{m_{\rho}}{m_{\pi \pi}}\right)^{4}$, as mentioned above.

Above $6 \mathrm{GeV}$, a diffraction picture describes the data best, with the $\rho$ decay angular distribution such that it niay agree with the strong absorption model mentioned above. At energies above $\approx 6 \mathrm{GeV}$, the $\mathrm{p}^{+} \pi^{-}$final state appears to be completely dominated by $\rho^{\circ} p$ production, with othcr resonant $\pi^{+} \pi^{-\mu}$ states assigned an upper limit of $0.2 \mu \mathrm{b}$. 2. Blechschmidt et. aI. (37) at DESY used a Iargc mannet-spark uhariber system in conjunction with a photon tagging facility to study $\rho^{\circ}$ production. They were thus able to have information on incoming photon energies good lu $\pm 50 \mathrm{MeV}$. Taking lata flum 3."' lu $4 . y \mathrm{GeV}$ on three target materia.1s (H, $\mathrm{C}, \mathrm{Al}$ ), theyr otudied the $\mathrm{A}$ dependence of the diffraction-type bahavius. Illeir results indicate: They f'ind $\rho$ masses of $\sim 770 \mathrm{MeV}$, including the $\left(\frac{\mathrm{m}}{\mathrm{m}_{\pi \pi}}\right)^{4}$ factor, with a width of order $120 \mathrm{MeV}$. Fittims the dala to the form $\frac{d \sigma}{\lambda t}=a e^{b t}$, they find a uependence on the mass number $A$ of

$$
\begin{aligned}
& a \cdots A^{1.68} \\
& b \sim A^{2 / 3}
\end{aligned}
$$

confirming the diffraction picture. For a completely opaque nucleus, we would expect $a \sim A^{4 / 3}$ while a transparent nucleus would lead to $a \sim A^{2}$. Assuming that the exponential can be used to determine the total cross-section, they quote 


$$
\sigma_{\text {tot }}(\gamma \mathrm{p} \rightarrow \mathrm{pp})=14.6 \pm 1.8 \mu \mathrm{b}
$$

3. Asbury et al (38) (DESY - Columbia) used a two-arm spectrometer to look for the charged $\pi$ pairs from the process

$$
\gamma+\mathrm{A} \rightarrow \mathrm{A}+\pi^{+} \pi^{-}
$$

using target materials $\mathrm{A}=\mathrm{Be}, \mathrm{C}, \mathrm{Al}, \mathrm{Cu}, \mathrm{A}, \mathrm{Pb}$. Studying forward production with their highly selective detection system, they found no $\pi^{+} \pi^{-}$enhancement other than the $\rho^{\circ}$ at invariant masses between 0.35 and $1.2 \mathrm{GeV} / \mathrm{c}^{2}$. According to the diffraction model, the forward coherent production cross-section for nuclei can be written as

$$
\left.\frac{d \sigma}{d \Omega}\right|_{O}=C(A) \times p_{\rho}^{2} \times f^{i}\left(p_{\rho}\right) \times f_{T}\left(R, t, \sigma_{\rho N}\right)
$$

where $\mathrm{C}$ is a constant, $\mathrm{f}^{\prime}$ is proportional to the total $\pi \mathbb{N}$ crosssection $\sigma_{\pi \mathbb{N}}$, and $f_{T}$ is a function of the nuclear radius $r=r_{0} A^{1 / 3}$, the momentum transfer, and the oN total cross-section. If we assume coherent production in the forward direction, and a uniform nuclear density inside a sphere of radius $R$, we can extract the nuclear radius $r_{0}$ and the pN total uruss-deotion $\pi_{\text {plN }}$ from the experimental data. We just have to measure $\frac{d \sigma}{d t}$ as a function of one of the known dependencies on $A, t$, or $p$, the others being kept constant. Fig. 2.2-6 shows the A dependence (normalized to $\mathrm{Be}$ ), at three energies, of the cross-section per nucleon. The resulting best values for $r_{o}$ and $\sigma_{\rho N}$ are

$$
\begin{aligned}
& r_{0}=1.29 \pm 0.4 \mathrm{f}, \\
& \sigma_{\rho N}=31.7 \pm 2.3 \mathrm{mb} .
\end{aligned}
$$




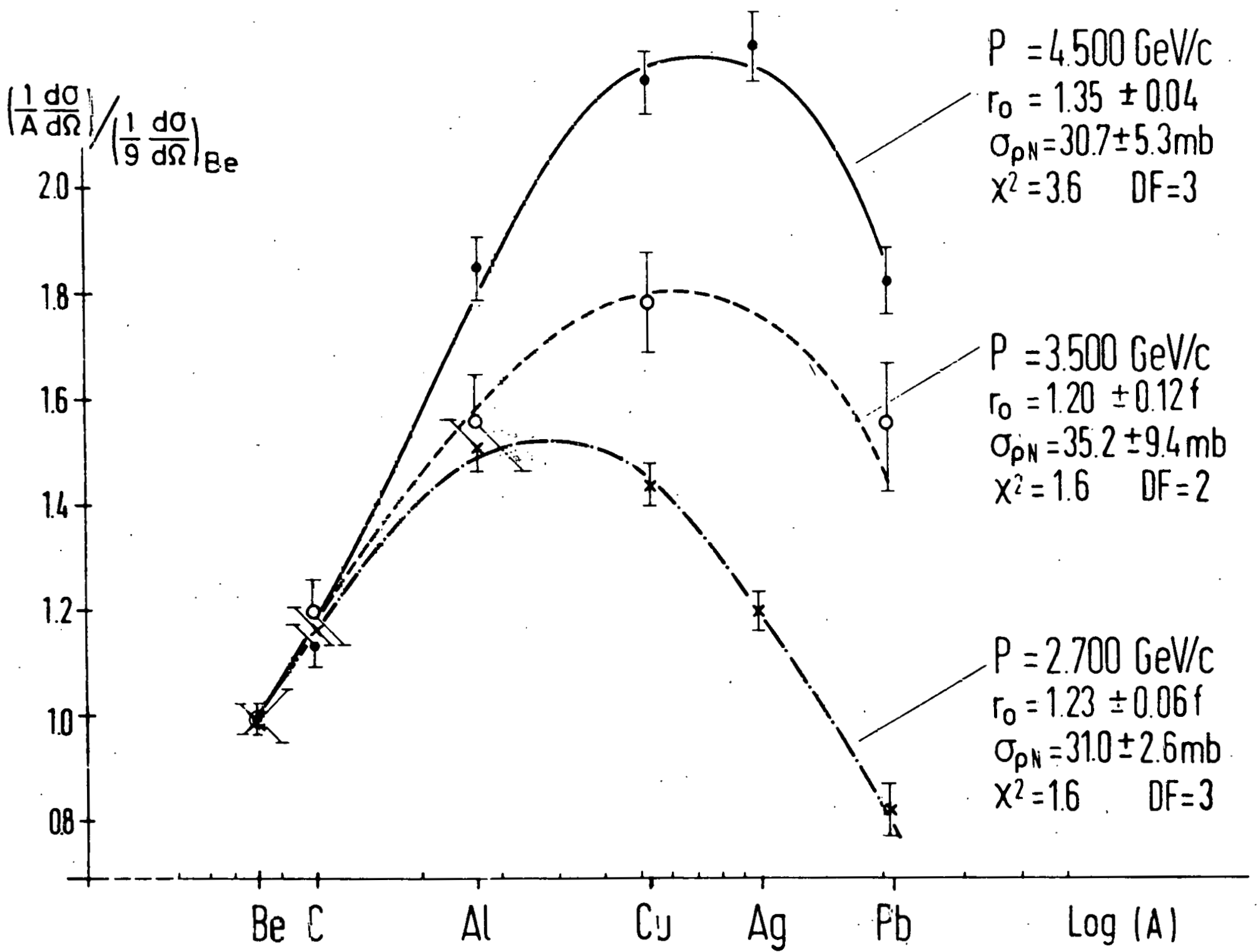

Fig. 2.2-D Coherent phcicproduction of $\rho^{0}$ mesons: Dependence of iccrward differential cross-section per nucleon $D$ the mass number A. Parameters $r$, $\sigma_{\text {oN }}$ result from fits according to Drill and Trefil. (From Ref. No. 30) 
This value for $\sigma_{\rho N}$ compares well with the $\pi \mathbb{N}$ total cross-section, which in the quark model should be the same: at these energies, $\sigma_{\pi \mathbb{N}} \approx 30 \mathrm{mb}$. The momentum transfer dependence is given in Fig. 2.2-7), for various elements. Again, the diffraction character appears. There may even be diffraction minima and maxima indicated outside the forward peak. Correcponding optical-model calculations by Fleischer ${ }^{(39)}$ have given good agreement. The bottom graph of Fig. 2.2-7 shows the ratio of the $\pi \mathbb{N}$ and $\rho \mathrm{N}$ forward cross-sections to be constant as a function of momentum.

The forward peak, deccribed by $\frac{d \sigma}{d t}=a e^{b t}$, again yields a $b(A)$ dependence of $b=b_{0} A^{2 / 3}$ (cf. eq. 2.2-6), with $b_{0}=10.5$. Since the diffraction model appears to give credible agreement with experimental features, the forward prediction was also used to get a check on the $\pi \pi$ mass distribution

$$
\frac{d^{2} \sigma}{d \Omega d m}=C(A) 2 m R(m) f_{T} f^{\prime}\left(p_{\rho}\right) p_{\rho}^{2}
$$

or, at high energy and small $\mathrm{l}$,

$$
\frac{d^{2} \sigma}{d \Omega d m} \approx C(A) 2 m R(m) p_{\rho}^{2} e^{a(t)}
$$

The resulting mass spectra are shown in Fig. 2.2-8, where the best fits again involve the mass-skewing factor $\left(\frac{m_{\rho}}{m_{\pi \pi}}\right)^{4}$, a Breit-Wigner distribution, and an empirical background. Best values for the resulting mass and width are $m_{\rho}=765 \pm 5 \mathrm{MeV}, \Gamma_{0}=130 \pm 5 \mathrm{MeV}$. The width is smaller than the 140 - $150 \mathrm{MeV}$ quoted as bubble chamber results $(19)$. 


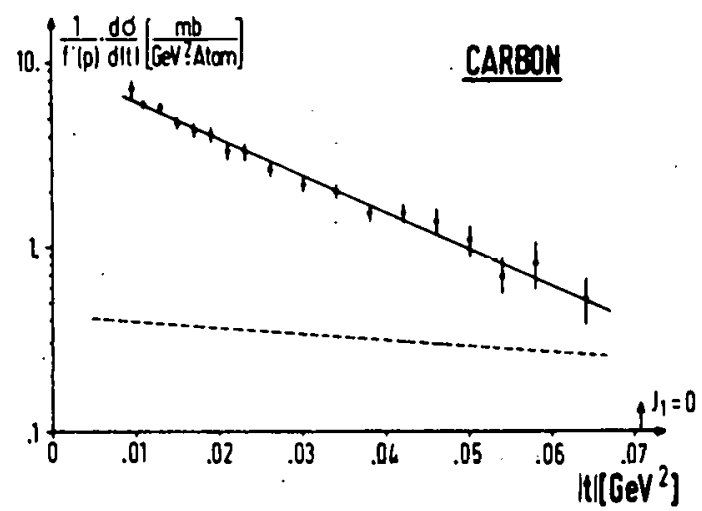

a)

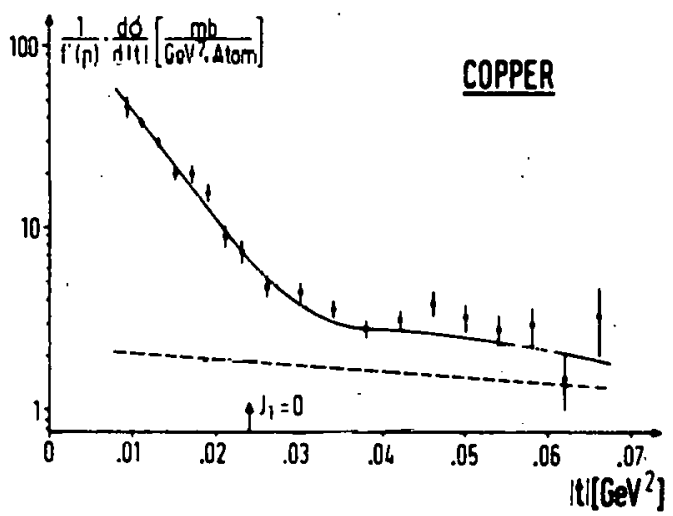

b)

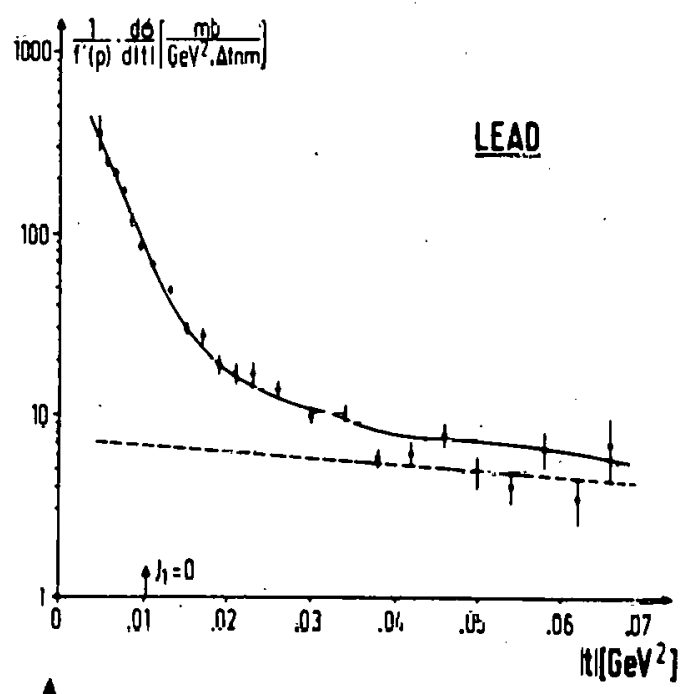

c)

ปี

它

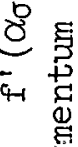

$\circ$ 品

दे

$\uparrow$

¿

도용

() कृ

of

in

o

ક્才ુ

r. 0 过

.

艺. I

क्ष

势然

的

出 8 宫

का है।

का .

0. 它

¿

ช

द

W

至

8 오

4.

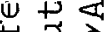

ij 0

त्र 40

4 a

$+\pi$

计

具 :

겅 을

व

过

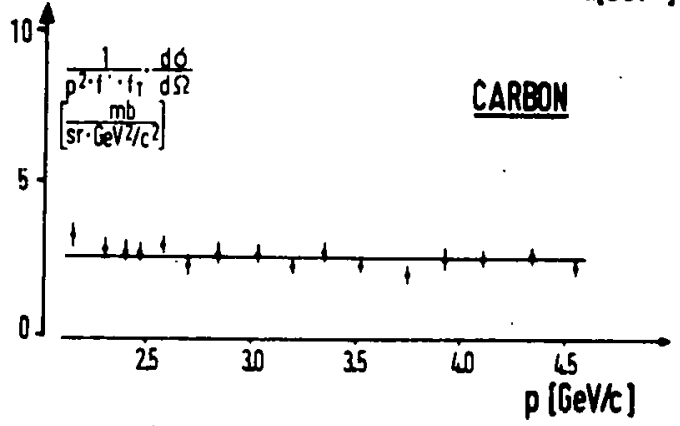

d)

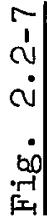



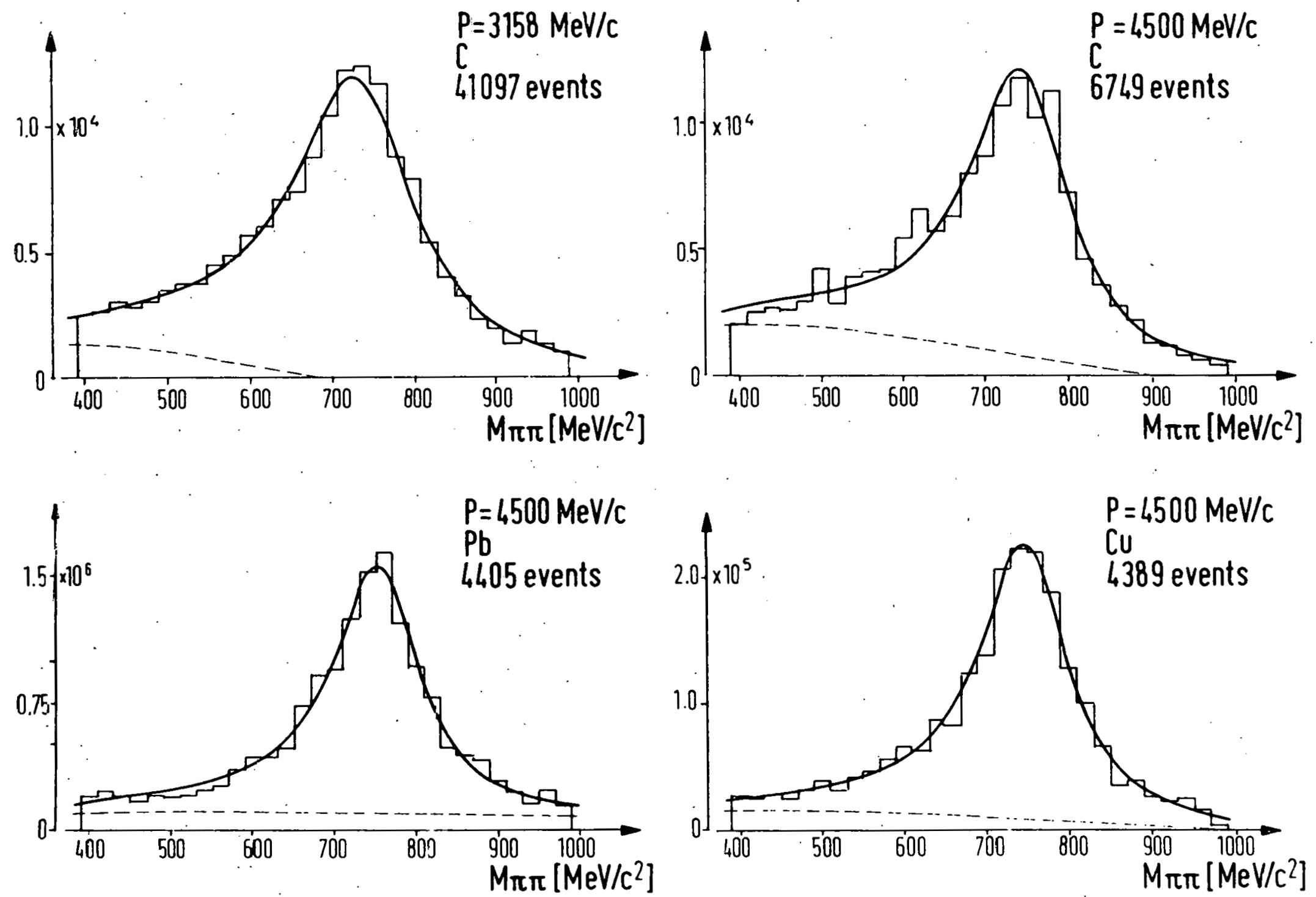

Fig. 2.2-8 $\pi^{+} \pi^{-}$invariant mess spectra for various target elements and momenta. Fits according to eq. 2.2-12. Dashed curves indicate estimates of non-resonant backgrounds. (From Ref. No. 38) 
4. Recently, at the Stanford Linear Accelerator, Jones et.al. (40) (40) have used a single-arm spectrometer to look at excitation functions for information on the photoproduction of neutral mesons. With the good flux and bad duty cycle characteristics of the Linac, this approach becomes desirable if difficult.

In the process $\gamma \mathrm{p} \rightarrow \mathrm{pm}^{\circ}$, precise momentum analysis of the finalstate protuil will yield information on the mass spectrum for $\mathrm{m}^{\circ}$. If we hold everything constant but vary the angle subtended by the spectrometer, a typical excitation function like in Fi.g. 2.2-9 results. As mentioned for the $K \Lambda-K \Sigma^{\circ}$ case on pagc $\Sigma \vartheta$, a two-body final state $\left(m^{\circ}\right.$ sharp) will show up as a sharp step, within the resolution of the detection system. Resonances of finite width, and three-or-more-body final states, put less of a kinematical constraint on the detected prnton, and will show up ao more grantulal rises. Fig. 2.2-9 shows that throe dire backyround protons below the lo'west production threshold (for the $\pi^{0}$ ). There is a $\pi^{0}$ step at the appropriate threshold value, a strong rise associated with $\rho^{0}$ juvaluction (from which we cannot separate the $\omega^{\prime} s$ ); we also expect steps superimposed at the $\eta$ and $\Phi$ thresholds, although thay do nul det'initely show up here. This metliod 18 open to errors stemming from background fitting, and the proper way to pick resonance parameters. The quoted results for photoproduction of $\beta^{\circ}$ on protons are the following, between 6.5 and $17.8 \mathrm{GeV}$ : Taking $\mathrm{m}_{\rho}=770 \mathrm{MeV} / \mathrm{c}^{2}$, $\Gamma_{\rho}=125$ from their best fits, they found that. inclusion of the massskewing factor did not improve these fits. Fig. 2.2-10 shows their 


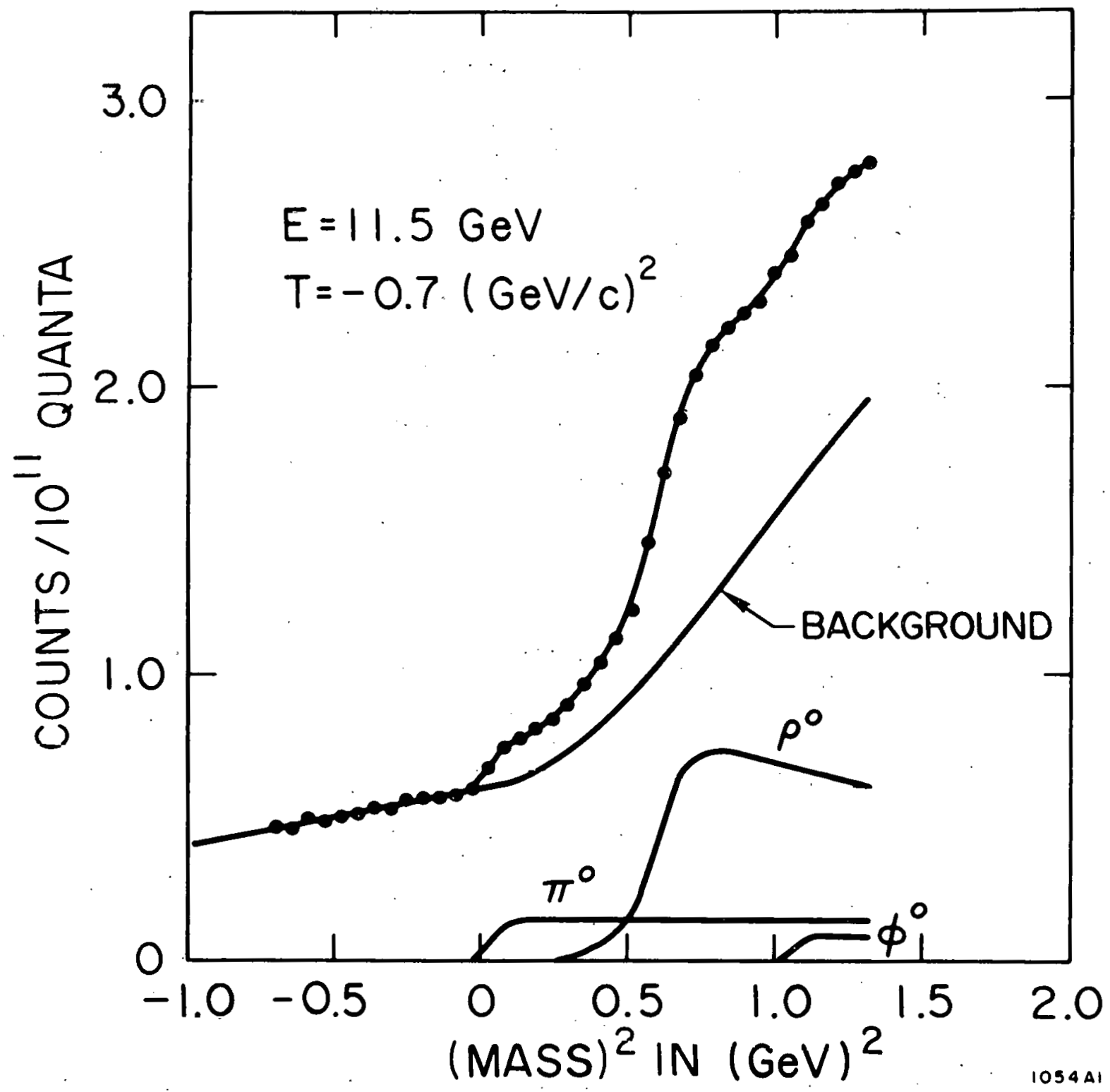

Fig. 2.2-9 Excitation function for the process $\gamma \mathrm{p} \rightarrow \mathrm{pX}^{0}$. Couriting rates of proton recoils, at given $\mathrm{E}_{\gamma}$ and $t$, plotted vs. mass ${ }^{2}$ of produced boson. (Frow Ref. No. 40) 
differential cross sections $\frac{d \sigma}{d t}$ between momentum transfer values $0.2<-t<0.9(\mathrm{GeV} / \mathrm{c})^{2}$. Evidently these results are consistent with the diffraction picture, with a non-shrinking diffraction peak going as $e^{8.5 t}$. At energies lower than $10 \mathrm{GeV}$, there may be some non-diffractive admixture, since between $t=0.7$ and $0.9(\mathrm{GeV} / \mathrm{c})^{2}$ there is a factor-of-two drop in cross-section between 6.5 and $10 \mathrm{GeV}$; if so, it dies out quickly and is no longer visible at $\sim 10 \mathrm{GeV}$. (Recall that we expect OPE contributions to decrease as $1 / k^{2}$ ).

It has to be kept in mind that the measured cross-section has to be extracted from the data through a difficist.t fitting procedure, where a typical error of $\pm 30 \%$ is quoted for the background shape (see Fig. 2.2-9). In addition, because of the non-separation of $\rho$ and $\omega$, an SU(.3)..sugGcsted pratio $\sigma(\rho) / \sigma(\omega)=9$ was aodumed, so that the apparent yield was simply reduced by 10\%. Parametcrs fur Iits according to $\frac{d \pi}{d t}=a e^{b t+a t^{2}}$ ars quuled in Flg. 2.2-10.

We have seen, in conclusion, that on the whole, $\rho^{\circ}$ photoproduction appears to be well described in terms of the diffraction pisture. Rclevant cxperimental numbers have been sumarizen in Table I.

\section{$2.3 \omega$ Photoproduction}

Due to the throemudy decay of the $\omega$ with a neutral in the final state, we have no counter experiments detecting the $p \omega$ final state. Not even the one-arm spectrometer, excitation function method is applicable, because the more copious production of $\rho^{\prime}$ s masks $\omega$ production within the present resolution of such systems. In principle, this could be overcome by measuring the G-parity violating $\omega \rightarrow \pi^{+} \pi^{-}$ 

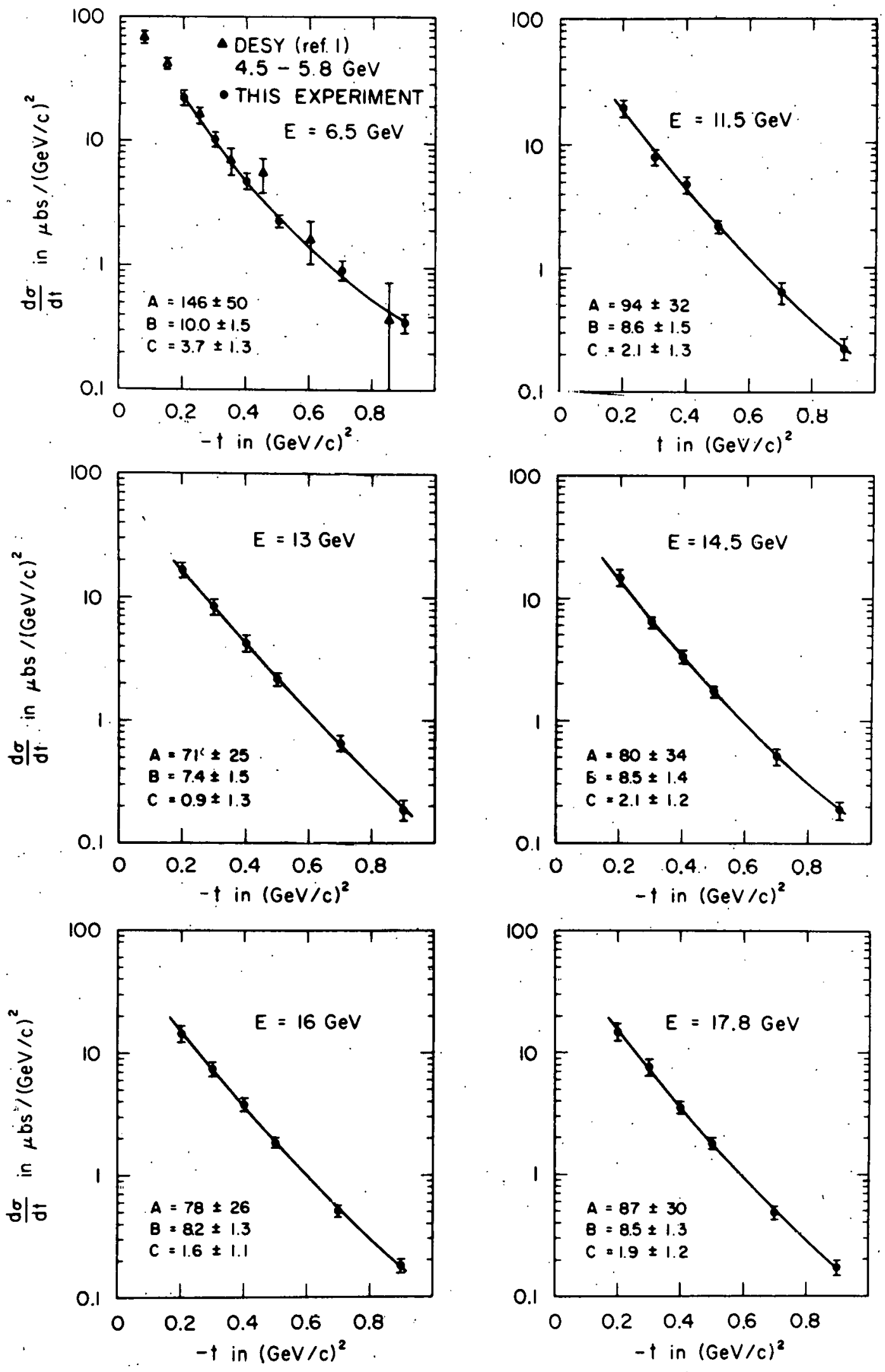

Fig. 2.2-10 High-energy photoproduction of $\rho^{\circ}$. Differential croso-cections fitted according to $\frac{d \sigma}{d t}=a e^{b t}+c t^{2}$. (From Ref. No. 40) 
mode which has a branching ratio of less than $1 \%$. No such data exist, but a double-arm spectrometer like that of reference (38) may soon be used for a similar investigation; however, the expected rates are very low. The bubble chamber results on $\omega$ photoproduction are shown in Figs. 2.3-1 and 2.3-2. They show, for the total cross section, a sharp rise above threshol. sloping off toward a value of $\sim 3 \mathrm{mb}$, not displaying the constant cross-section behavior expected for diffraction production, but rather a picture suggestive of a considerable OPE contribution (which we expect to decrease with $\sim 1 / k^{2}$ ). The angular dependence $\frac{\mathrm{d} \sigma}{\mathrm{dt}}$ displays a forward peak and a more isotropj.c component, with the peak becoming more pronounced with increasing energy. The best fit to the total cross-sertion datid has an OPE-type tierm and a ulit'raction-like term with very sma.1.1 energy-depenience

$$
\sigma_{\text {tot }}(\gamma p \rightarrow p \omega) \approx A E_{\gamma}^{-1.6}+\mathrm{BE}_{\gamma}^{-0.08}
$$

The OPE exponent -1.6 is, according to a compllation by Morrioon (42), typical of many $N \mathrm{PE}$ processur, and the diffraction-term has an exponent taken from $\rho$ production.

A thorough inveatigation of this OPE-plus-diffretiun piclure Led to a value for the radiative decay width of the $\omega$ of $\Gamma(\omega \rightarrow \pi \gamma)=$ $(0.7 \pm 0.3) \mathrm{MeV}$. Parameters for the exponential dependence $\frac{d \sigma}{d t}(t) \alpha e^{b t}$ are again given in Table $I$.

Using eq. 2.3-1, we can tentatively separate out the diffractive contribution to $\sigma_{\text {tot }}(\gamma \mathrm{p} \rightarrow \mathrm{p} \omega)$. For $\mathrm{k} \lesssim 6 \mathrm{GeV}$, we find $\sigma_{\text {diff }}(\gamma \mathrm{p} \rightarrow \mathrm{p} \omega)$ $\approx 1.7 \pm 0.9 \mu \mathrm{mb}$. Note that this gives good agreement with the ratio. 


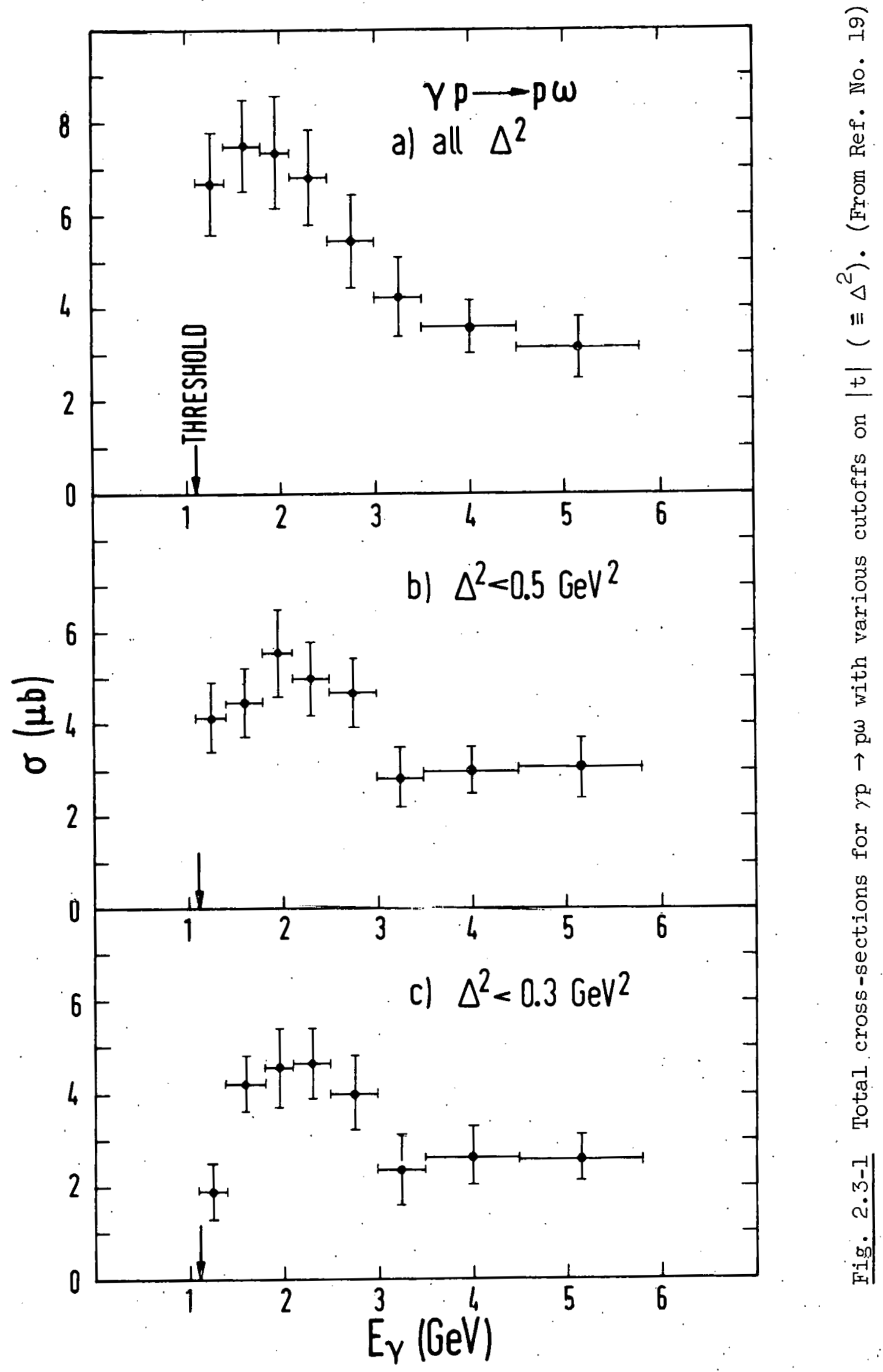




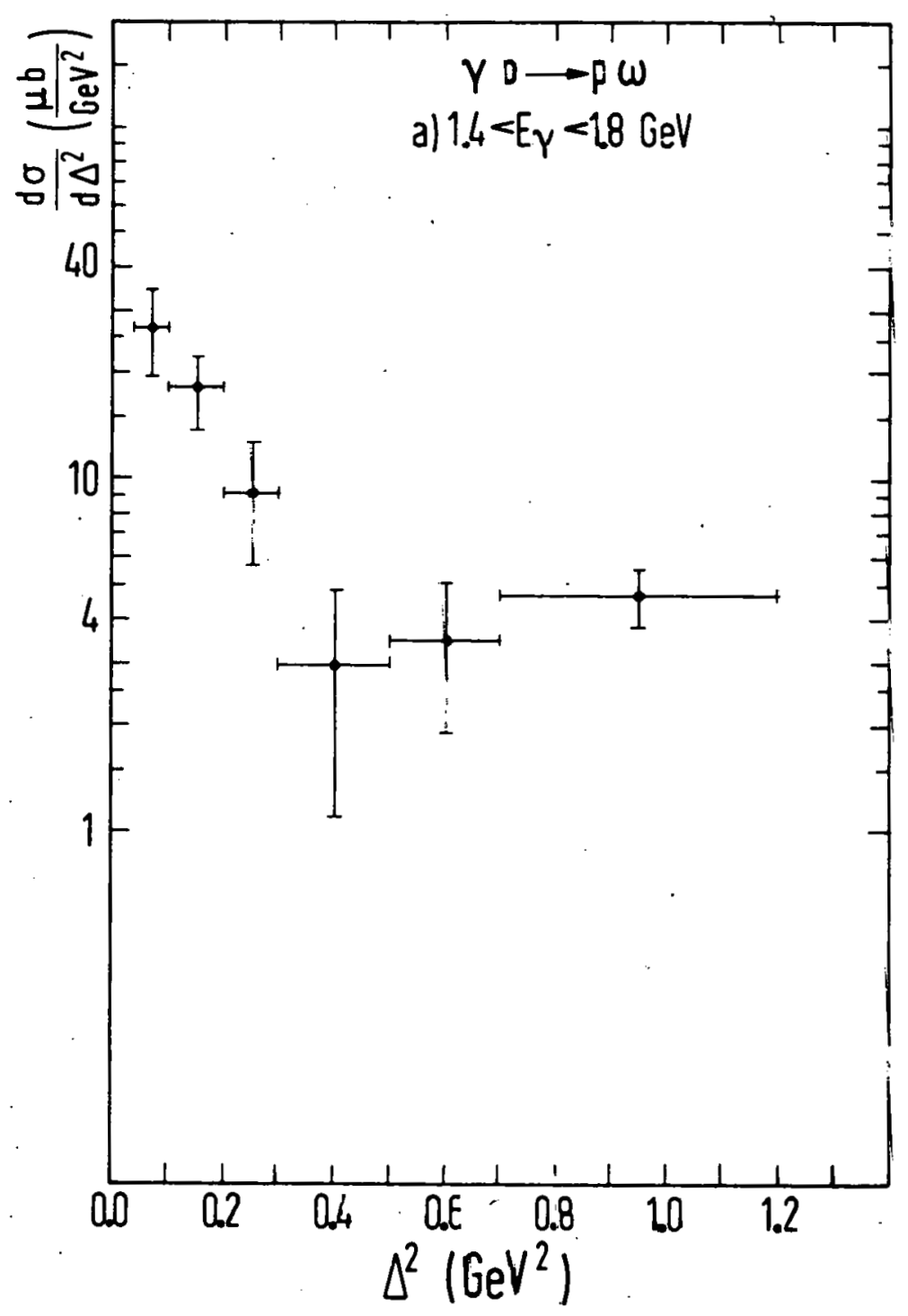

Fig. 2.3-2(a) Differentiel cross-section. $\frac{\mathrm{d} J}{\mathrm{dt}}\left(\gamma \mathrm{F} \rightarrow \mathrm{p} \omega^{\prime}\right)$ for $1.4 \leq \mathrm{k} \leq$ $1.8 \mathrm{Ger} / \mathrm{c}$. (From Ref. No. 19)

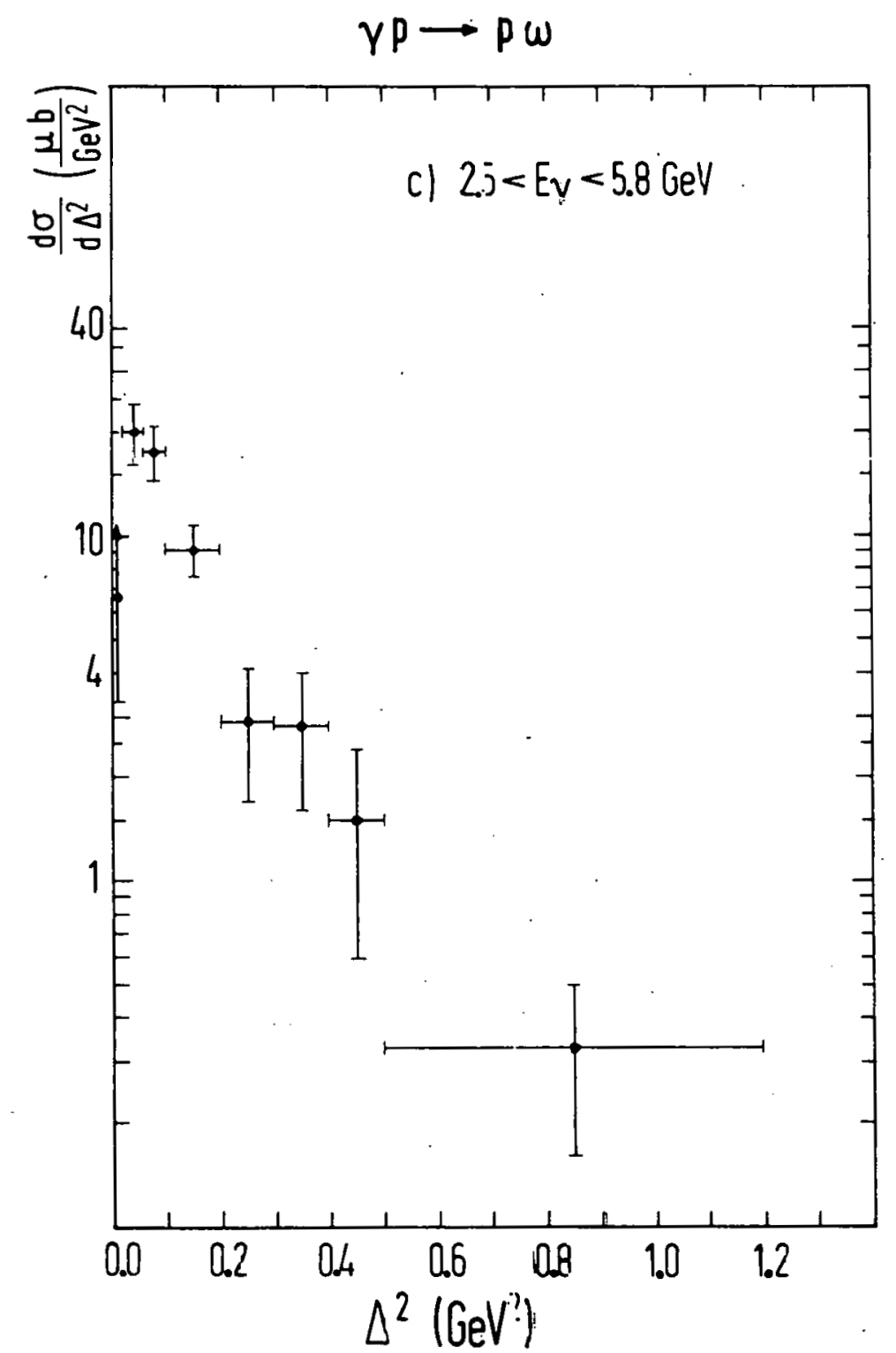

Fig. 2.3-2(b) Differential cross section $\frac{d \sigma}{d t}(\gamma \mathrm{p} \rightarrow \underline{\omega})$ for $2.5 \leq k \leq$

$5.8 \mathrm{GeV}$; C. (From Ref. No. 19) 
$\sigma_{\text {diff. }}(\gamma p \rightarrow p \omega) / \sigma_{\text {diff. }}\left(\gamma p \rightarrow p \rho^{\circ}\right) \approx 1 / 9$ predicted by the diffraction picture combined with the usual SU(3) assumptions (eq. 2.1-9)。

\section{4 $\Phi$ Photoproduction.}

The detection of $\Phi$ mesons can in principle proceed along precisely the same lines as that of $\rho^{\prime} s$, since the dominant decay mode is $\mathrm{K}^{+} \mathrm{K}^{-}(\sim 47 \%)$ with the added feature that $\Gamma_{\Phi} \ll \Gamma_{\rho}$, so that the background subtractions do not lead to serious problems. However, the $\Phi$ gets photoproduced only at a very small rate, so that there are comparatively few data. The bubble chamber results up to $\sim 6 \mathrm{GeV}$ are given in Figs. 2.4-1 and 2.4-2. The resulting features, within the errors quoted, are these: the total cross-section $\gamma \mathrm{p} \rightarrow \mathrm{p} \Phi$ does not rise as sharply at threshold as that for $\rho$ or $\omega$ production. No structure is discernible, possibly due to poor resolution and statistics. $\frac{\mathrm{d} \sigma}{\mathrm{dt}}$ is compatible with diffractive behavior, especially for the high-energy interval, with a best fit $\frac{d \sigma}{d t}(t) \alpha e^{(3.5 \pm 0.9) t}$, a slope much smaller than what we find for $\rho$ and $\omega$ photoproduction. Asbury et al. (43) at DESY have used thelr double-arm spectrnmeter to detect $\mathrm{K}^{+} \mathrm{K}^{-}$pairs in the same manner as $\pi^{+} \pi^{-}$pairs from $\rho$ decay, measuring $\Phi$ forward coherent production on nuclei. Mass distributions are given for $\mathrm{K}$ pairs in Fig. 2.4-3. For all elements studied, they are seen to be strongly peaked in the mass region of the $\Phi\left(1020 \mathrm{MeV} / \mathrm{c}^{2}\right)$. Analysis of the A dependence similar to that for the $\rho$ case (section 2.2) yielded a total cross section $\sigma_{\Phi \mathrm{N}} \approx 13.4 \mathrm{mb}$. In addition, a study of the decay angular distribution yields a result consistent with a purely transverse polarization of the $\Phi$ mesons. 


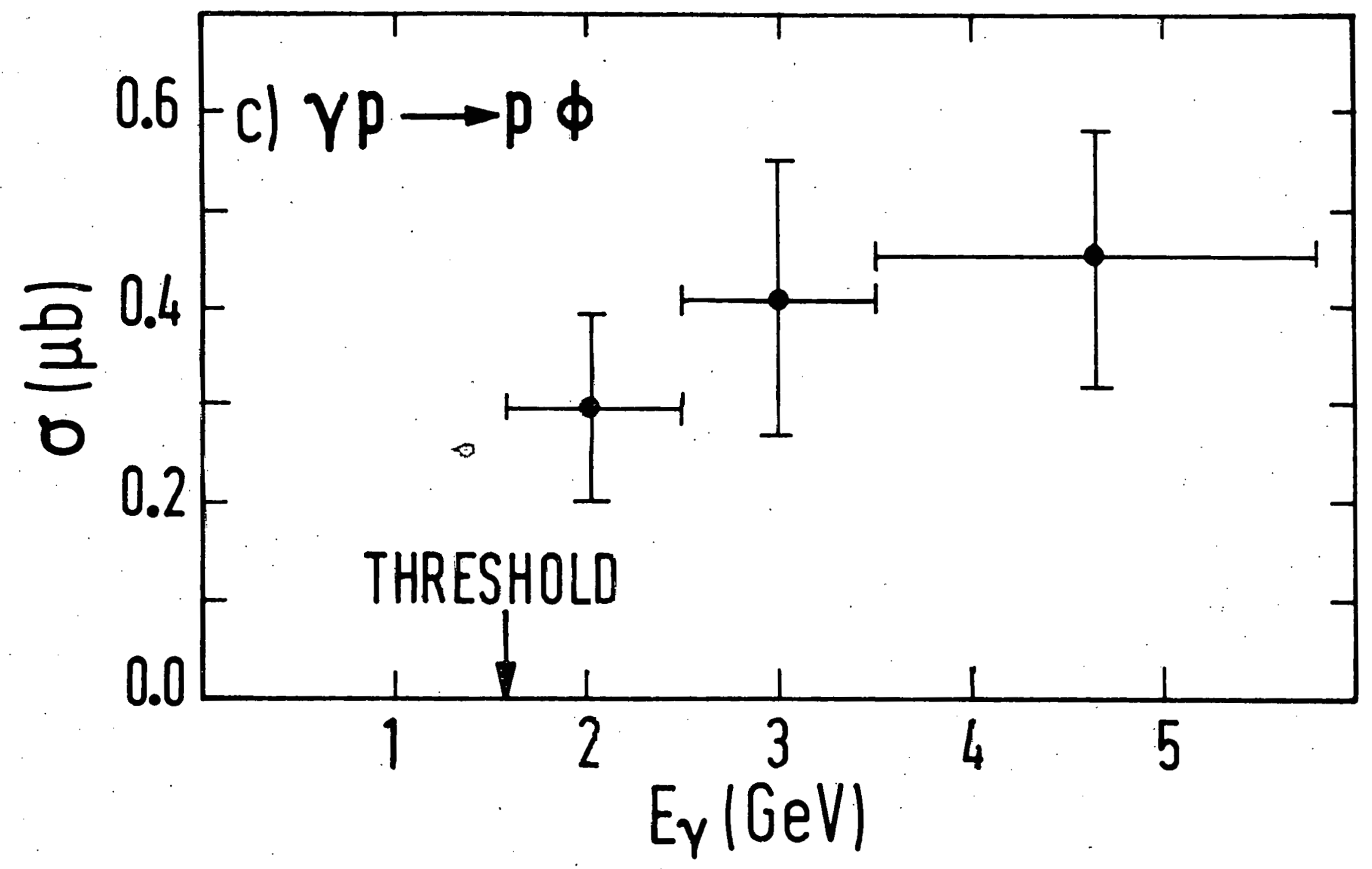

Fig! 2.4-1 Total cross-section for $\gamma p \rightarrow p \Phi$. Bubble chamber data. (From Ref. No. 19) 

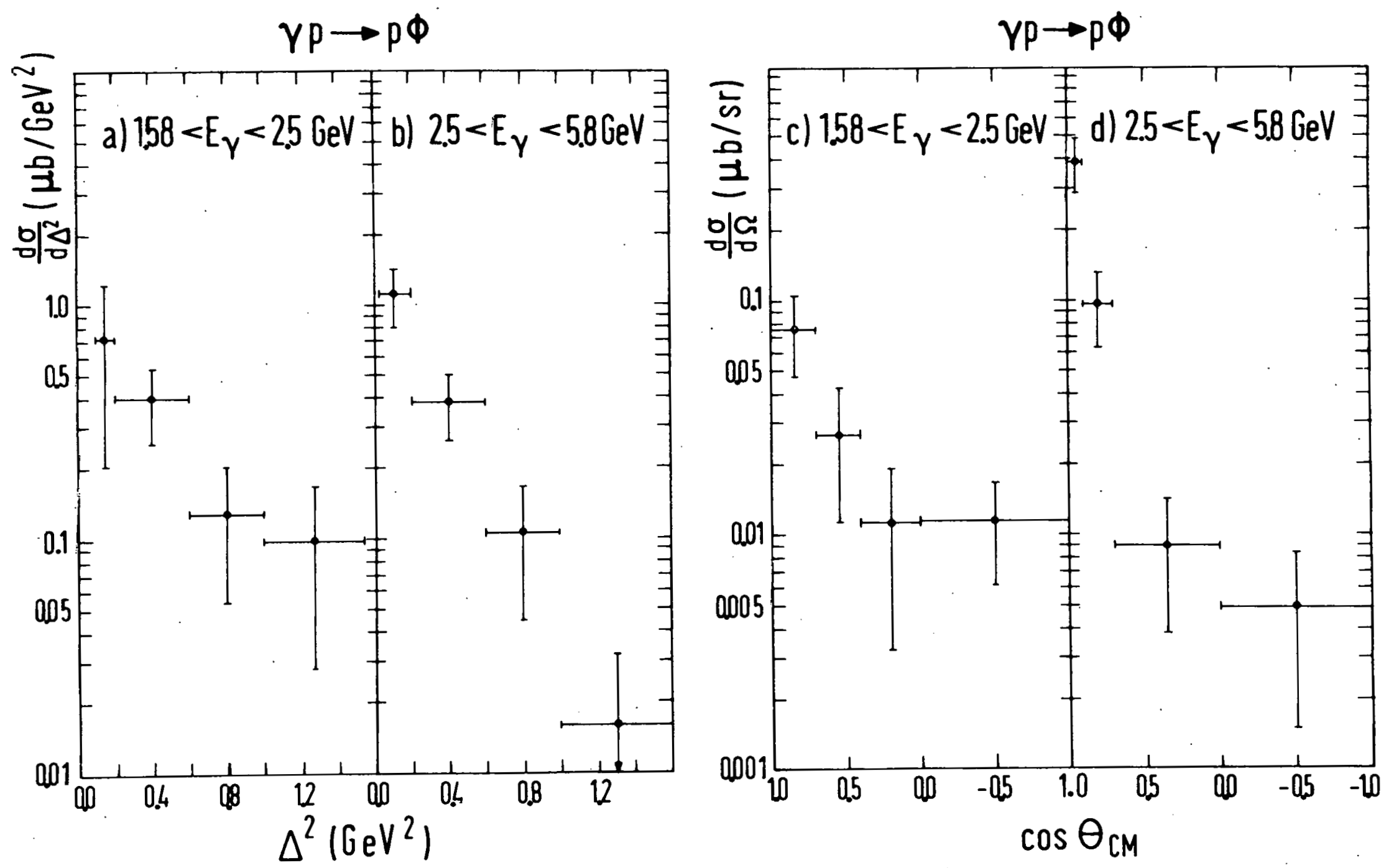

Fig. 2.4-2 Differential cross-sections for $\gamma p \rightarrow p \Phi$ for two energy intervals. (From Ref. No. 19) 
Photoproduction of meson on complex nuclei

$P_{Q}=5.2 \mathrm{GeV} \quad \theta_{6}=0^{\circ}$
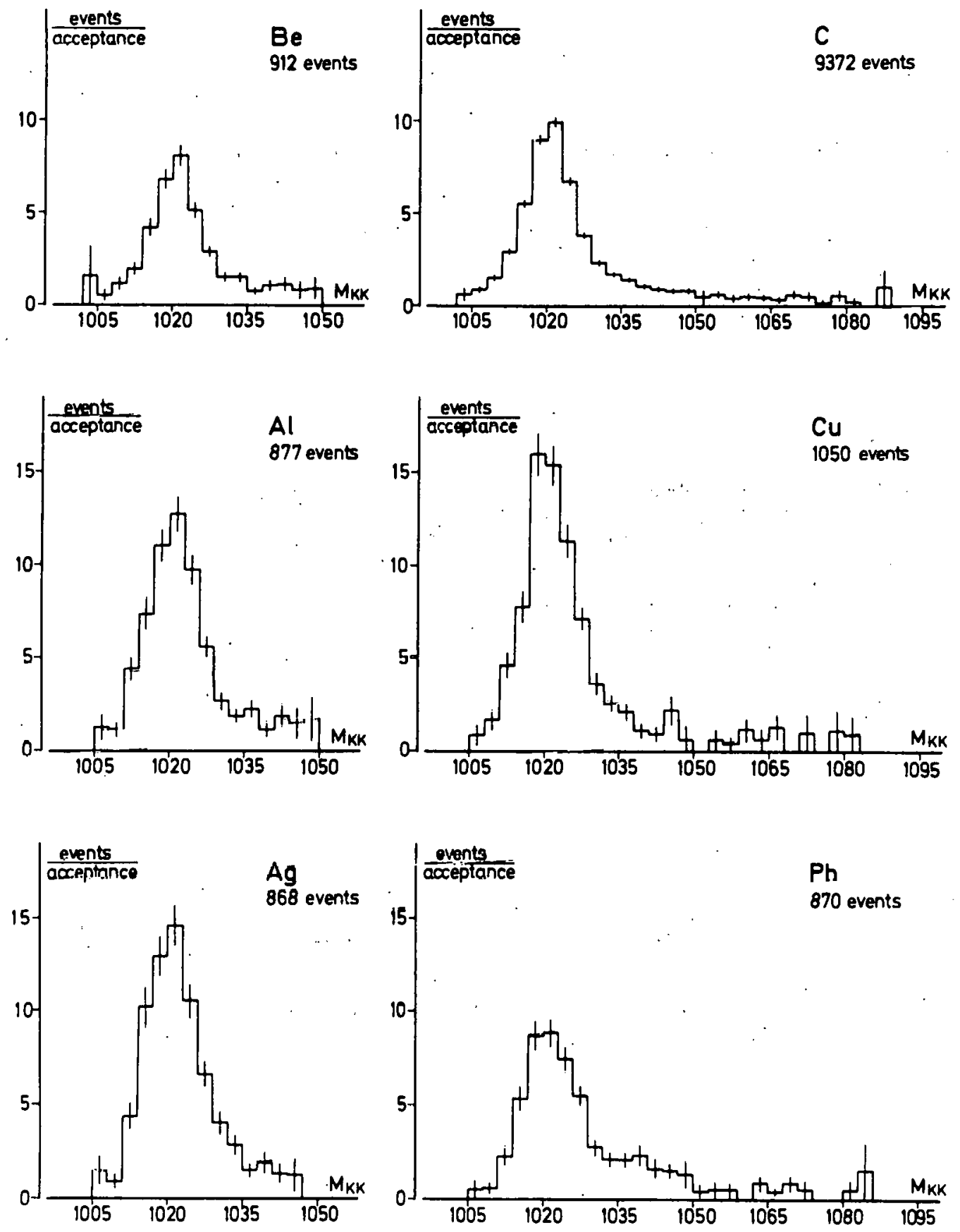

Fig. 2.4-3 $\mathrm{K}^{+} \mathrm{K}^{-}$invariant mass spectra for various target elements. (From Ref. No. 43) 
The extension of the data to higher energies is particularly important for a study of the diffraction mechanism. Two of the beam survey experiments at SIAC were used to study the production of $\mathrm{K}^{+}$close to the forward direction, on $\mathrm{Be}^{(44)}$ and hydrogen targets ${ }^{(45)}$. Relating the $\mathrm{K}$ yield to the $\Phi$ production cross-section by assuming that all K's stem from $\Phi$ decays, upper limits were given for $\sigma(\Phi)$. Using various assumptions on backgrounds and $t$ dependence, $\sigma(\Phi)$ at 7-8 GeV was estimated to be between 0.5 and $1.0 \mu \mathrm{b}$.

The recent single-arm experiments of Jones et al. (40) looking for excitation functions in single-neutral-meson production observed clearly discernible steps in the yield function (see Fig. 2.2-9) at $\Phi$ threshold. By following a suitable subtraction procedure, they quote a cross-section behavior according to $\frac{d \sigma}{d t}(\gamma p \rightarrow p \Phi) \approx a e^{b t}$ with $b \approx 4.5(\mathrm{GeV} / \mathrm{c})^{-2}$. Their measured values are at $|t|$-values $>0.3(\mathrm{GeV} / \mathrm{c})^{2}$ only, so an extrapolation to small t may be problematic. However, if we believe the quoted $t$ dependence as governing the entire cross-section behavior, then we can use the forward amplitude and the optical theorem to estimate the total cross-section for $\Phi \mathbb{N}$ scattering (see next section) from the vector dominance model; the resulting values are $\sigma_{\text {tot }}(\Phi p)=9.7 \pm 2.0 \mathrm{mb}$ at $13 \mathrm{GeV}, 7.2 \pm 1.9$ at $16 \mathrm{GeV}$. Together with the $6 \mathrm{GeV}$ values of $\sim 13.4 \mathrm{mb}$ quoted by Asbury et al. (43), this look's like a definitely decreasing trend, in disagreement with expectations of the diffraction model. (Fig.2.4-4). 


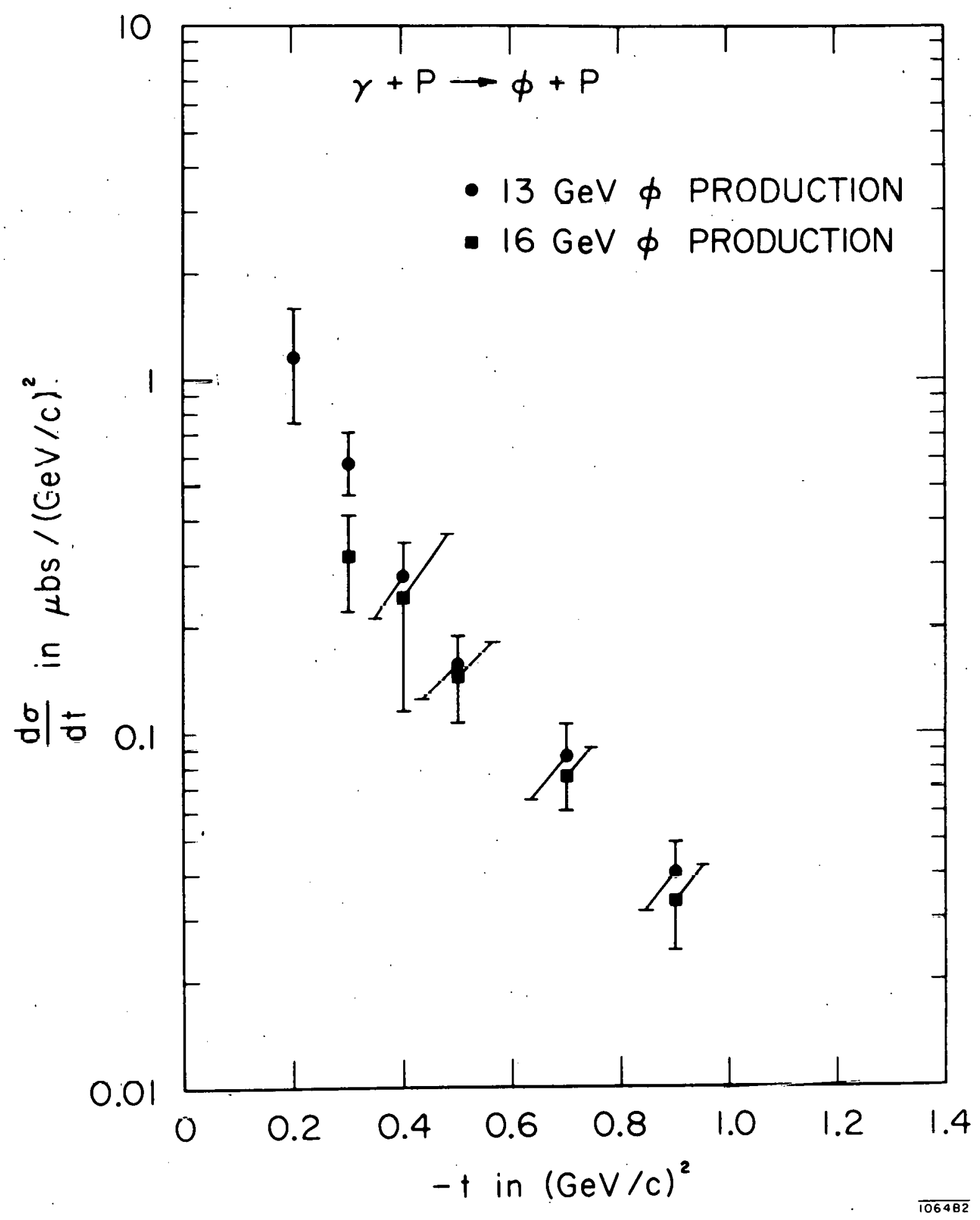

Fig. 2.4-4 Differential cross-sections for $\gamma \mathrm{p} \rightarrow \mathrm{p} \Phi$ at high energies. (From Ref. No. 40)' 


\subsection{The Vector Dominance Model}

The experimental information on vector meson photoproduction is briefly summarized in Table $I$. If we go back to the considerations of section 2.1 and try to decide on the dominant mechanisms for the process $\gamma p \rightarrow \mathrm{pV}^{\circ}$, we find that (if, for the time being, we leave isobar formation out of the picture), neither the OPE nor the diffraction model yields satisfactory comparisons. The OPE prediction (2.1-5) that $\sigma(\omega) \approx 9 \sigma(\rho)$ is obviously not satisfied. But the fit (2.3-1) to the $w$ data suggests that there is an OPE contribution for the $w$ which accounts for the distinctly non-diffractive behavior of $\omega$ production at lower energies. If we separate out the presumable diffraction part, the diffraction model prediction (2.1-9) $\sigma(\rho): \sigma(\omega)$ : $\sigma(\Phi)=9: 1: 2$ does appear to reproduce the $p / \omega$ ratio reasonably. However, the observed $\Phi$ rate is much too low (by a factor of order 10 ). Let us see how we can patch up this discrepancy. The predicted ratio from the graph

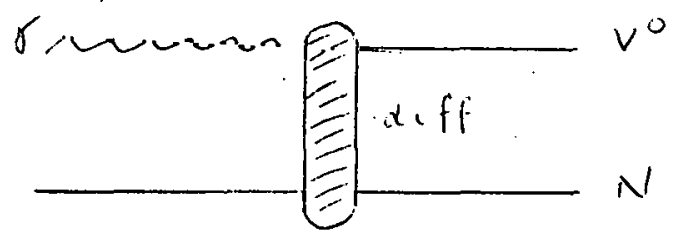

assumed SU(3) symmetry, and $\mathrm{P}$ trajectory exchange, where $\mathrm{P}$ is a unitary singlet of $\mathrm{J}^{\mathrm{P}}=0^{+}$. Meshkov and Ponzini (46) argue in the rramework of the collinear group $\mathrm{SU}(6)_{\mathrm{W}}$ that high energy forward elastic scattering data make the unitary singlet exchange illusory, and that higher representations have to be exchanged. Their method has an attractive feature that is not an ad hoc construction, but fails to 
reproduce the $\Phi$ rate by a factor of 2 .

The most obvious remedy is to introduce symmetry breaking. This comes most naturally in the framework of the vector dominance model, where we write the above graph somewhat differently, introducing a direct photon-vector meson coupling:

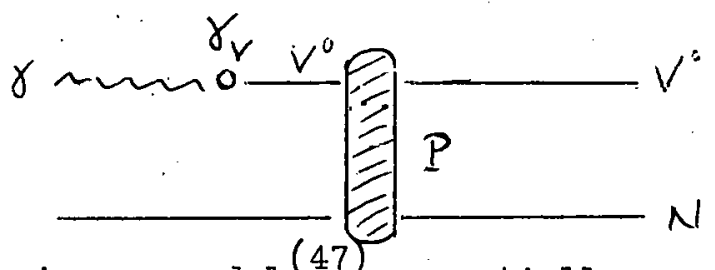

The vector dominance model $(47)$ essentially assumes that we can write the hadronic electromagnetic current as a linear combination of phenomenological vector meson currents

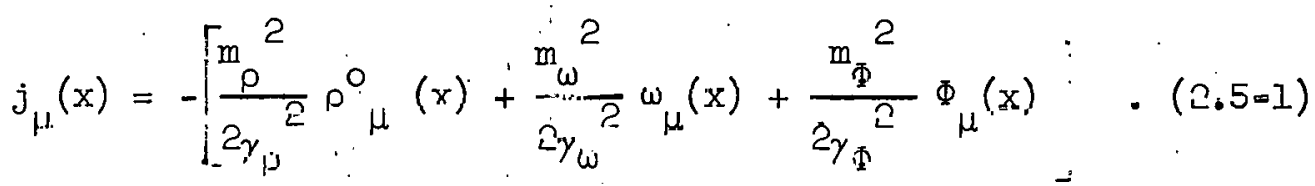

With the usidal SU(3) assumptions we cun then write the incident. plioton as

$$
|\gamma\rangle=\frac{\sqrt{3}}{2}\left|\rho^{\circ}\right\rangle+\frac{1}{3}|\omega\rangle-\frac{\sqrt{2}}{3}|\Phi\rangle \mid
$$

which gives us a relation between photon-indured and vector-mesoninduced reactions according to

$$
\sigma(\gamma a \rightarrow b c)=\sum_{i} \frac{\alpha_{\pi}}{\gamma_{i}^{2}} \sigma_{t r}\left(V^{O} a \rightarrow b c\right)
$$

(where $\sigma_{\text {tr }}$ means that we specify helicity states \pm 1 for the vector mesons.)

We now apply eq. 2.5-3 to the diffraction process $\gamma p \rightarrow p V^{\circ}$, noting that we can write the direct $\gamma \mathrm{V}^{\circ}$ coupling in terms of the 
leptonic decay widths

$$
\Gamma\left(\mathrm{V} \rightarrow \mathrm{e}^{+} \mathrm{e}^{-}\right)=\frac{\dot{\alpha}^{2}}{12} \frac{4 \pi}{\gamma_{\mathrm{V}}^{2}} \mathrm{~m}_{\mathrm{V}}+\text { small terms }
$$

Table I shows the experimental information on the leptonic decay widths. We then write the critical ratio.

$$
\frac{\sigma(\gamma p \rightarrow \Phi p)}{\sigma\left(\gamma p \rightarrow \rho^{\circ} p\right)}=\frac{m_{\rho}}{m_{\Phi}} \frac{\Gamma\left(\Phi \rightarrow e^{+} e^{-}\right)}{\Gamma\left(\rho^{0} \rightarrow e^{+} e^{-}\right)} \frac{\sigma_{e l}(\Phi p)}{\sigma_{e l}\left(\rho^{o} p\right)}
$$

with the recent results from $\mathrm{DESY}^{(48)}$ on the $\Phi \rightarrow \mathrm{e}^{+} \mathrm{e}^{-}$decay width of $1.1 \pm 0.2 \mathrm{eV}$ and the accepted $\rho \rightarrow \mathrm{e}^{+} e^{-}$width of $\sim 6 \mathrm{keV}$. We then insert, using the optical theorem,

$$
\frac{\sigma_{e l}\left(\Phi_{p}\right)}{\sigma_{e l}\left(\rho_{p}{ }_{p}\right)}=\left|\frac{\sigma_{\text {tot }}\left(\Phi_{p}\right)}{\sigma_{\text {tot }}\left(\rho^{\circ} p\right)}\right|^{2}
$$

the $\sigma_{\text {tot }}$ values from the preceding chapter or Table $I$, and find all quantities in (2.5-5) experimentally determined. The numerical cheok is:

$$
\text { Left-hand side } \approx \frac{1}{40} \quad \text { Right-hand side } \approx \frac{1}{60}
$$

Preliminary numbers of $2.1 \pm 0.9 \mathrm{keV}$ for the radiative decay width of the $\Phi$, as reported by a CERN group $(49)$, or of $1.30 \pm 0.21 \mathrm{keV}$, as deduced from an $\mathrm{e}^{+} \mathrm{e}^{-} \rightarrow \Phi$ experiment at the Orsay electron storage ring $(101)$, improve the numerical agreement somewhat. (Recall the SU(3) prediction $\Gamma\left(\rho \rightarrow \mathrm{e}^{+} \mathrm{e}^{-}\right): \Gamma\left(\omega \rightarrow \mathrm{e}^{+} \mathrm{e}^{-}\right): \Gamma\left(\Phi \rightarrow \mathrm{e}^{+} \mathrm{e}^{-}\right)$ = 9: 1: 2: or, with some symmetry-breaking introduced into the $\gamma \mathrm{V}$ vertex $(50), 9: 0.65: 1.30)$ 
Let's recapitulate then: we essentially keep SU(3) symmetry in the $\gamma \mathrm{V}$ vertex, introduce symetry-breaking in the VVP vertex, where the $P$ is still a unitary singlet. This symetry-breaking can easily be introduced in terms of the quark model (51): from the relations for the total cross-sections

$$
\begin{aligned}
& \sigma(\rho p)=\sigma(\omega p)=\frac{1}{2}\left(\sigma\left(\pi^{-} p\right)+\sigma\left(\pi^{+} p\right)\right), \\
& \pi\left(\Phi_{p}\right)-2 \sigma\left(K^{+} p\right)+\sigma\left(\pi^{-} p\right)-2 \sigma\left(\pi^{+} p\right),
\end{aligned}
$$

where we insert experimental results on the RHS, we obtain $v(\rho p)=v(\omega p)=28 \mathrm{mb}, v(\Phi p)=11.5 \pm 1.5$, in fair agreemerit with the alrect experimental numbers quoted in Table $I$.

If we try to play the same game with $\omega$ production, we have the difficulty that only two preliminary (and disorepant) muluer's exist for $\Gamma\left(\omega \rightarrow \mathrm{e}^{+} \mathrm{e}^{-}\right)$: a CERN experiment ${ }^{(19)}$ on $(1) \rightarrow \mathrm{e}^{+} e^{-}$giving $a$ value of $0.49 \pm 0.19 \mathrm{keV}$, and the Orsay storage ring experiment (IOI) $e^{+-} e^{-} \rightarrow \omega$, yielding $\Gamma\left(\omega \rightarrow e^{+} e^{-}\right)=1 . I \pm 0.25 \mathrm{keV}$. Moreover, it is not trivial to extract the diffraction part from the total crosssection for $\gamma p \rightarrow p \omega$. Thkine the "beat guess" values (see Table I), we find

$$
\frac{\sigma(\gamma p \rightarrow p \omega)}{\sigma\left(\gamma p \rightarrow p \rho^{\circ}\right)}=\frac{m_{\rho}}{m_{\omega}} \frac{\Gamma\left(\omega \rightarrow e^{+} e^{-}\right)}{\Gamma\left(\rho^{0} \rightarrow e^{+} e^{-}\right)} \quad\left(\frac{\sigma_{t o t}(\omega p)}{\sigma_{t o t}\left(\rho^{0} p\right)}\right)^{2}
$$

The first and third factors on the RHS are $\approx I$ (in the absence of direct information on $\sigma_{\text {tot }}(\omega \mathbb{N})$, we take the quark model value), and we find 
Lefthand side $=\frac{1}{10} \quad$ Righthand side $=\left\{\begin{array}{l}\frac{1}{13} \text { taking CERN value for } \Gamma\left(\omega \rightarrow e^{+} e^{-}\right) \\ \frac{1}{6} \text { taking ORSAY value for } \Gamma\left(\omega \rightarrow e^{+} e^{-}\right)\end{array}\right\}$

In view of the poor input material, we will not take this as a strong endorsement. Still, the VDM appears to make the overall experimental picture of photoproduction of vector mesons consistent with our present theoretical underslanding; at modprate energies $(<6 \mathrm{GeV})$. Before we worry about the apparent decrease of the $\Phi$ cross-section above $6 \mathrm{GeV}^{(40)}$, let us wait for more complete data.

2.6 Some Specific Tests of the Vector Dominance Model.

Without wanting to prejudge other attempts to account for the initially inconsistent picture in $\Phi$ photoproduction, we take the apparent success of the VDM as an indication for the relevance of eq. (2.5-3). The only direct test of the direct coupling hypothesis would be a comparison of decay modes like $\omega \rightarrow \pi \gamma$ and $\omega \rightarrow \pi \rho$ (which does not work energy-wise); $A_{1} \rightarrow \pi \gamma, A_{1} \rightarrow \pi \rho$ (where the experimental number is not clear, and we have to disentangle transverse and longitudinal $\rho^{\prime} s$ ); or $A_{2} \rightarrow \pi \gamma, A_{2} \rightarrow \pi \rho$ (where the $\mathrm{J}^{\mathrm{P}}=2^{+}$value allows only transverse $\left.\rho^{\prime} s\right)$. We would predict the radiative decay width to be $\sim 1 \mathrm{MeV}$; unfortunately, no direct experimental information exists on this decay. Some recent analysis of somewhat less direct experimental evidence has yielded a consistently favorable picture.

We can, for instance, analyze photoproduction cross-sections and check for consistency with $\pi$ production of vector mesons:

$$
\gamma \mathrm{p} \rightarrow \mathrm{N} \pi \leftrightarrow \overline{\pi p} \rightarrow \mathrm{V}^{\circ} \mathrm{n}
$$


The corresponding amplitudes are connected by the VDM model and timereversal invariance, if we separate out the proper helicity and isospin amplitudes. Dar et al. ${ }^{(52)}$ have shown several combinations of cross-sections which are accessible to such checks. Fig. 2.6-1 shows the agreement between the two sides of the rellation

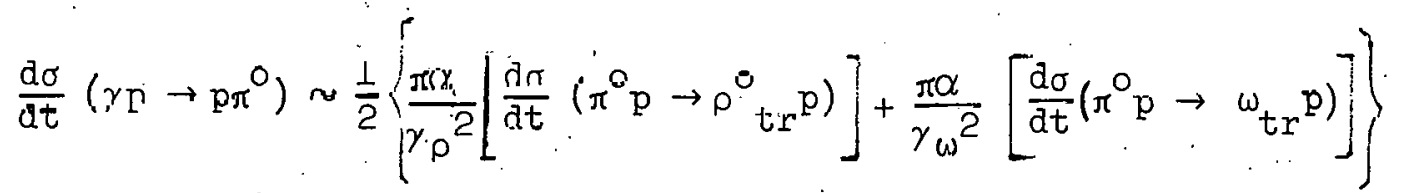

where the $\pi^{\circ}$-initiated amplitudes on the RHS can be obtained by isospin invariance from $\pi^{-}$and $\pi^{+}$data. A corresponding prediction for $\gamma n \rightarrow \pi^{\circ} n$ is drawn into the picture, and awaits experimental check.

A particularly suitable case is presented by a comparicon of lle reactions $\gamma p \rightarrow \pi^{+} n$ and $\pi^{-} p \rightarrow \rho^{n} n$

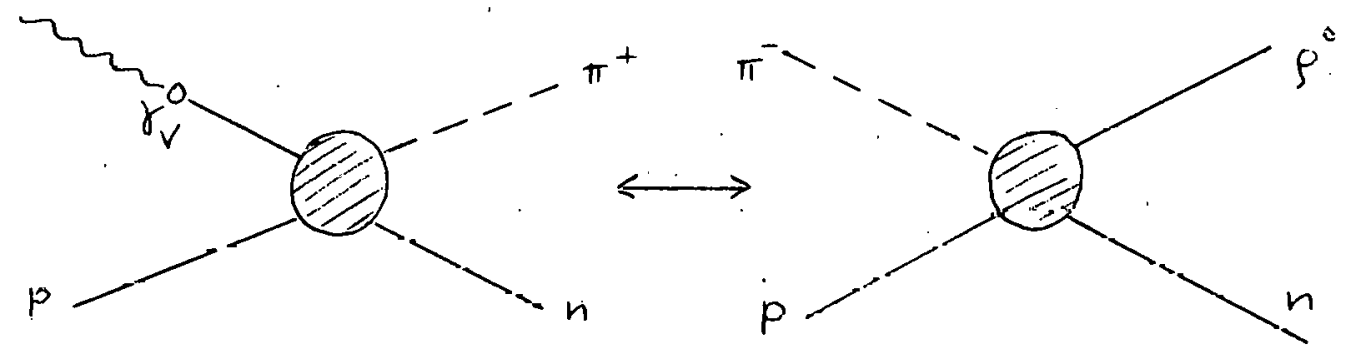

They are linked up by the VDM and by I and T invariance. A detailed comparison is poesithle for the data on either side of the relationship

$$
\frac{d \sigma}{d t}\left(\gamma p \rightarrow \pi^{+} n\right) \approx \frac{\pi \alpha}{\gamma_{\rho}^{2}} \rho_{I I}(t) \frac{d \sigma}{d t}\left(\pi^{-} p \rightarrow \rho^{0} n\right)+\text { w terms }+\underset{\text { interference terms }}{(2.6-3)}
$$

where $\rho_{11}(t)$ is the helicity density matrix element giving the fraction of transversely polarized $\rho$ 's (since we want to compare 


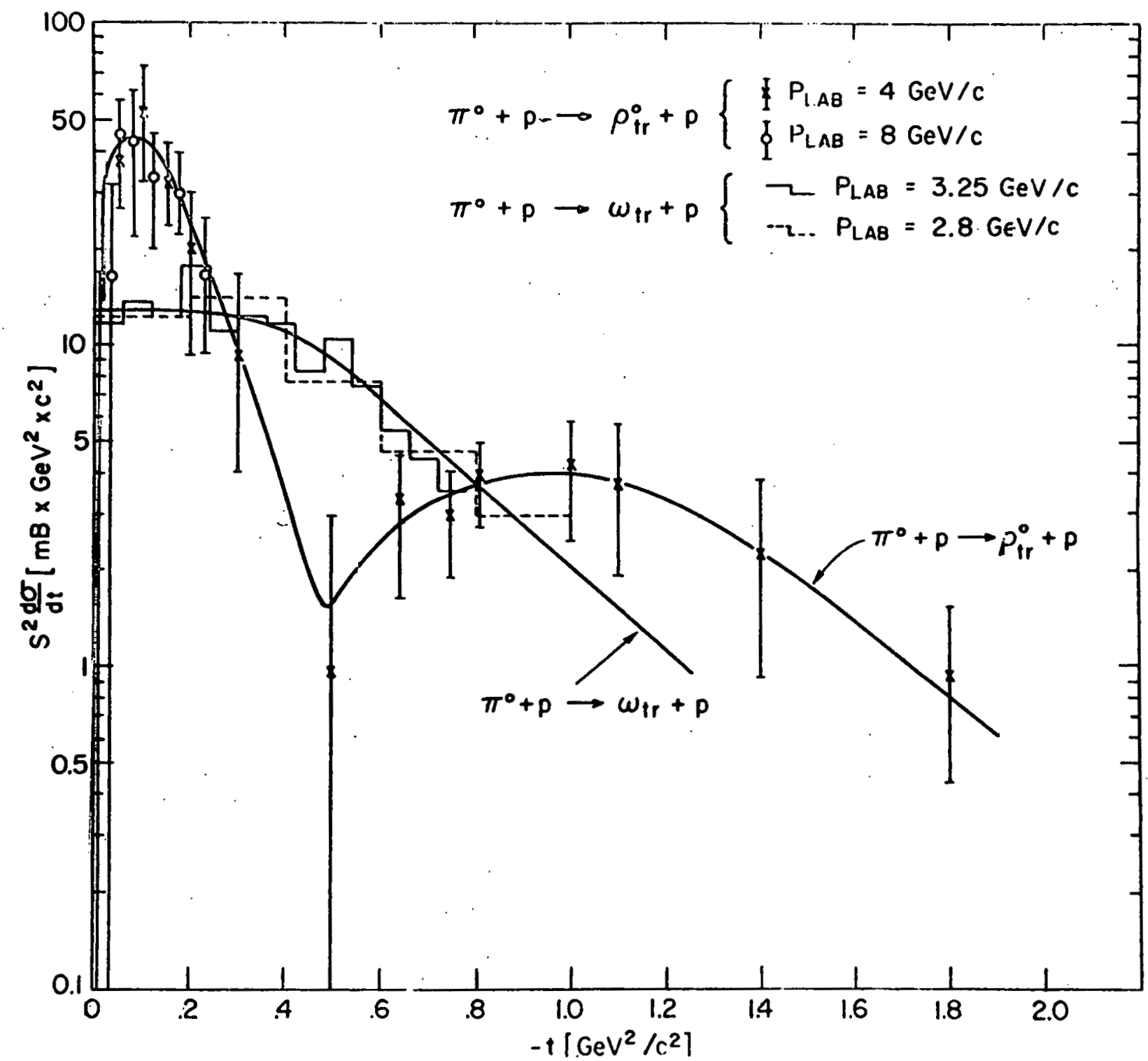

Fig. 2.6-1 Prediction for $\pi^{\circ}$ photoproduction (solid curve: off protons; dashed zurve: off neutrons) according to vector dominance model. Data points are for process $\gamma p \rightarrow p \pi^{\circ}$. (From Ref. No. 52) 
the same helicity states for photons and $\rho^{\prime} \mathrm{s}^{(53)}$ ). The material presented in section 2.5 suggests that we may disregard $\omega$ and $\Phi$; and evaluate the RHS with $\rho$ 's alone. Various authors $(54,55,102)$ have recently given quantitative evaluations of eq. (2.6-3) at several energies, carefully checking the helicity density matrix element $\rho_{1 l}$ and the background difficulties inherent in the RHS exper- . juntial तata. Fig. 2.C-乞 glves examples at 4 and $8 \mathrm{GeV} / \mathrm{c}$ from ref.(54): at small $t$, good agreement is apparent. The disagreement at larger $t$ can be accounted for by considering the interference effect of the contribution of the $\omega$ contribution to the vector line. Isospin invariance then implies that we should not use $\sigma\left(\gamma \mathrm{p} \rightarrow n \pi^{+}\right)$for the comparison, but rather a linear combination of cross sections which will make the interferense terms canccl. We llepefore recall that we can break up the photoproduction amplitudes into isnvertor- and icosoálar-photun lrittiated parts, $A_{E}$ and $A_{v}$. yielding

$$
\begin{aligned}
& \frac{d \sigma}{d t}\left(\gamma p \rightarrow \pi^{+} n\right)=\left|A_{v}+A_{s}\right|^{2} \\
& \frac{d \sigma}{d t}\left(\gamma n \rightarrow \pi^{-} p\right)=\left|A_{v}-A_{s}\right|^{?}
\end{aligned}
$$

and replace $\sigma\left(\gamma \mathrm{p} \rightarrow \mathrm{n} \pi^{+}\right)$by $\frac{1+\mathrm{R}}{2} \sigma\left(\gamma \mathrm{p} \rightarrow \mathrm{n} \pi^{+}\right) ; \mathrm{R}$ is the $\pi^{-} / \pi^{+}$ration, which is experimentally known frow $\pi$ photopioduclion oft deuterium (cf. section 3.6). Agreement then becomes quite satisfactory. Another teist of the VDM hypothesis was reported by. Asbury et a... (56), who use coherent vector production on nuclei to compare the two sides of

$$
\left.\frac{d \sigma}{d t}\left(\gamma A \rightarrow \rho^{O} A\right)\right|_{t=0}=\frac{1}{16} \frac{k}{p j} \frac{\alpha}{\gamma_{\rho}^{2}} \sigma_{\text {tot }}^{2}:(\rho A \rightarrow \rho A)
$$


$-83-$
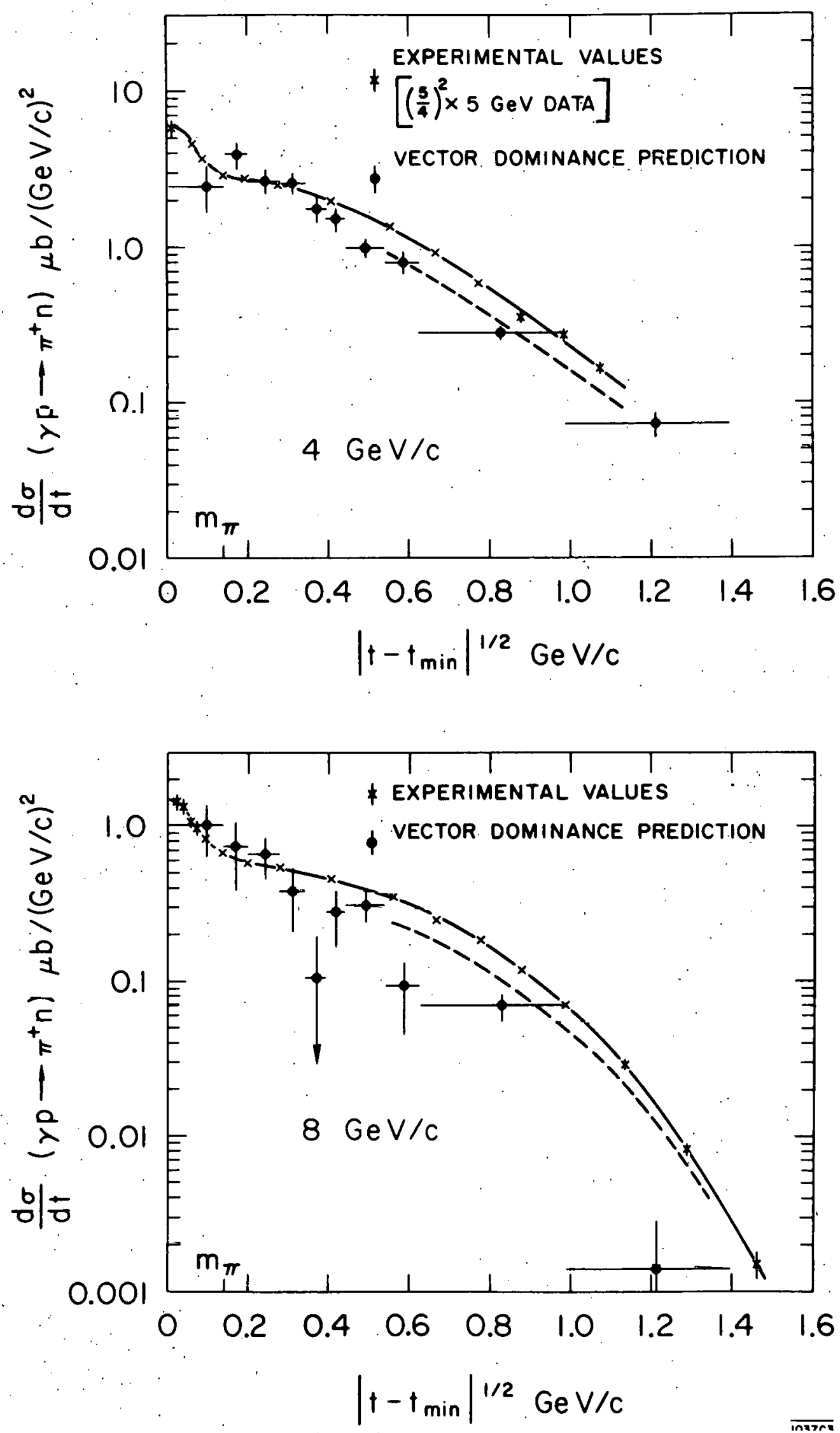

Fig. 2.6-2 Data check of $\pi^{+}$photoproduction cross-sections predicted by VDM from $\pi^{-} p \rightarrow \rho^{\circ}$ n data. Dashed curves incorporate correction due to $\rho \omega$ interference. (From Ref. No. 54) 
in order to check the validity of the VDM-suggested diagram

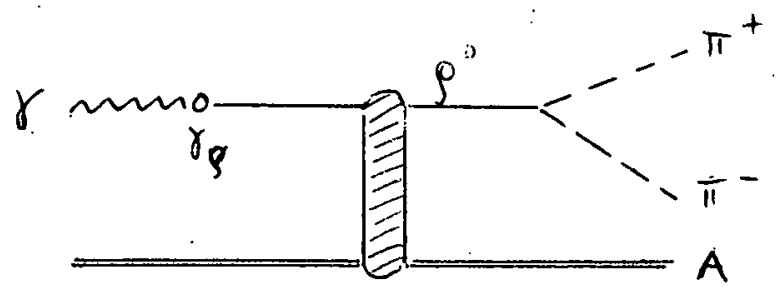

They studied the $\rho^{\circ}$ photoproduction croes ocction at smali t. values between 0.004 and $0.06(\mathrm{GeV} / \mathrm{c})^{2}$ and obtained the LHS of eq. (2.6-5) by extrapolating to $t \approx 0$. The RHS was calculated by computing $\sigma_{\text {tot }}(\rho A \rightarrow \rho A)$ from the total. $\rho N$ cross-sectim, as detemined from the relative photoproduction yield on various nuclei (38). The resulting numerical value for $\frac{\gamma_{\rho}^{2}}{4 \pi} \approx 0.42 \pm 0.1$ compares well with its direct determination from $\rho \rightarrow e^{+} e^{-}$decays and $e^{+} e^{-} \rightarrow \dot{\rho}$ annihilation. The good numerical agreement should then be taken as evidence for the validity of the graph otudied here.

A further application of the VIM was recently proposed by H. Joos (57) who suggests a comparison of nucleon isobar decays $\Delta \rightarrow \operatorname{INp}$ and $\Delta \rightarrow \mathbb{N} \gamma$. For the $I=3 / 2$ isobars $\Delta(1920)$ and $\wedge(2420)$ a. rough estimate for the $N \gamma \Delta$ vertex is possible from the structure observed at corresponding energies in $\pi^{\circ}$ hackward plotuproduction (68). Thus, estimates for the oN decuy widths are made according to

$$
\Gamma(\Delta \rightarrow \rho \mathrm{O})=K \frac{\alpha_{\pi}}{\gamma_{j}^{2}} \Gamma(\Delta \rightarrow \gamma \mathbb{N})
$$

where $\mathrm{K}$ is a kinematical factor. Correspondingly, the graph

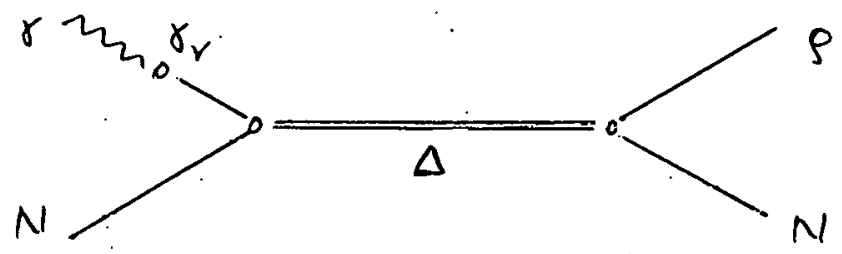


can be evaluated to give a value for the photoproduction cross-section at resonance peak energy (cf. Fig. 2.2-5). The cross-section for $\gamma \mathrm{p} \rightarrow \Delta(2720) \rightarrow \mathrm{p} \rho^{\circ}$ is $0.6 \mu \mathrm{b}$; the observed value, uncorrected for helicity +1 only, $2+1 \mu \mathrm{b}$. We regard this as a reasonable initial agreement. $(\triangle(1920)$ is too hard to separate uniquely out of OPE and diffraction contribuliuis).

\subsection{Total Cross Section $\sigma(\gamma \mathrm{p})$}

More tests of the VDM can be thought up. We will here stop with the treatment of the total electromagnetic cross-section for hadrons. We have, as mentioned in 2.2 and 2.4 , some experimental information on the cross-sections $v(\rho N)$ and $\sigma(\Phi N)$. We can use the VDM then to predict the total $\gamma$-hadronic cross-section.

The high-energy behavior of the photon-proton total cross-section is of considerable interest. In the high-energy limit, the forward Compton graph, when viewed as a simple diffraction process as discussed in the previous sections for veotor mesons,

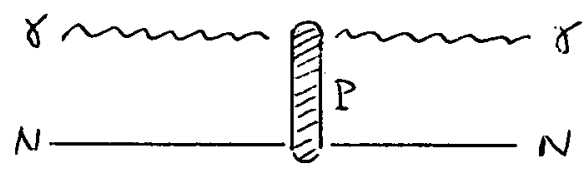

has to vanish for real photons. Suppose we have a right-handed photon coming in: the $P$ exchange graph will leave its hellclly, $\lambda_{k}-+1$, unchanged. When we now cross into the $t$-channel, the time-reversed photon remains right-handed, but its momentum is reversed; we then have combined incoming helic1ty $\lambda_{k_{I}}=+1-(-1)=+2$. The directchannel pole $P$ would have to carry two units of helicity. In simple 
Regge language (cf. section 3.3): - if $\sigma_{\text {tot }}(s \rightarrow \infty)=$ constant, we expect a "Regge pole" $P$ with angular momentum $\alpha_{P}(t=0)=1$, which behaves like a vector under three-dimensional rotations. It therefore cannot couple to two photons (just as a spin-one object cannot decay into two photons). Thus, the optical theorem will postulate $\sigma_{\text {tot }}(\gamma \mathrm{p}) \rightarrow 0$ as $\mathrm{E} \rightarrow \infty$.

If we want to salvage the situation, we can elther assume $\alpha_{\dot{P}}(0)<1$, which would lose the main reason why the "Pomeranchon" $P$ was invented; or we can invoke a residue function with a singularity at $t=0$ to cancel the "nonsense zero"; or we, can assume a t'ixed pole at $J=1$.

On the other hand, the forward production of vector mesons is, as we pointed out, also ascribed to $P$ exchange in the diffraclluil picture; fur the massive mesons, crossing into the t-channel does nnt lead to the helicity - 2 assignment fur the $\mathrm{P}$; the asymptotic crnss section for some inelastic processes is therefore not expected to be suppressed. In future high-energy experjments on $\sigma_{\text {tot }}(\gamma p)$, we will particularly have to try tis use photón beams of longitudinal as well as transverse polarization (e.g. using virtual photons from electroproduction) in order to understand the process; for helicity = 0 (virtual) photons, the nonsenve zero obviously does not exist.. If we want to make specific predictions for the magnitude of $\sigma_{\text {tot }}(\gamma p)$, we can use the VDM to link up op $\rightarrow$ anything and $V^{\circ} p \rightarrow$ anything. The most simple-minded prediction then neglects the $\omega$ and $\Phi$ parts of eq. 2.5-3, and we write 


$$
\sigma\left(\gamma_{\mathrm{v}} \mathrm{A} \rightarrow \mathrm{B}\right)=\frac{\alpha_{\pi}}{\gamma_{\rho}^{2}} \sigma\left(\rho^{\mathrm{D}} \mathrm{A} \rightarrow \mathrm{B}\right)
$$

where $\gamma_{\mathrm{v}}$ is an isovector photon. This leads to a prediction of $\sigma_{\text {tot }}\left(\gamma_{\mathrm{v}} \mathrm{p}\right) \approx 100 \mu \mathrm{b}$, taking numbers from Table $I$. The isoscalar contributions will be considerably smaller; in the energy region where data exist on $\sigma_{\text {tot }}(\mathrm{VN})$, from $3-5.5 \mathrm{GeV}$, we expect a value of $\sigma_{\text {tot }}(\gamma \mathrm{p}) \approx 110 \mu \mathrm{b}$ on these grounds.

Another estimate uses the VDM in the specific form of the diffraction dissociation model $(30)$, making use of the experimental information on the forward cross-sections, $\left.\sigma\left(\gamma p \rightarrow v^{O} p\right)\right|_{t=0}$. Inserting the best values into the relation $(58)$

$$
\sigma_{\text {tot }}(\gamma p)=\sqrt{16 \pi}\left[\frac{\mathrm{e}}{\gamma_{\rho}}{\frac{\mathrm{d} \sigma^{1 / 2}}{\mathrm{dt}}}^{1 / \rho}(\rho)+\frac{\mathrm{e} \sin \theta_{\mathrm{y}}}{2 \mathrm{f} y} \cdot \frac{\mathrm{d \sigma ^{1/2 }}}{\mathrm{dt}}(\omega)+\frac{\mathrm{e} \cos \theta_{\mathrm{y}}}{2 \mathrm{f}_{\mathrm{y}}} \frac{\mathrm{d} \sigma^{1 / 2}}{\mathrm{dt}}(\Phi)\right]
$$

where the weighted couplings have the mentioned ratio $9: 0.65: 1.3$. We again find a prediction of $\sigma_{\text {tot }}(\gamma p) \approx 100 \mu \mathrm{b}$. It is not easy to assign errors to these predictions, since we have to take the diffractive part of the forward cross-sections alone. They ought to be good to $\sim \pm 10 \%$.

\subsection{Measurcment of the Total Cross-Section $\sigma(\gamma p)$.}

The experimental problems involved in the measurement of $\sigma(\gamma p)$ are obvious; above all, it is a very difficult task to separate hadronic from purely electromagnetic events. Moreover, if we try to look at a.1. final-state particles in order to make up for the lack of initial-state information (due to the usually poor definition of 
photon energies), we min into the problems associated with the detection of neutrals $\left(\gamma, \pi^{\circ}, K^{\circ}, n, \ldots\right)$.

Therefore, one of the main experimental tasks will be an adequate definition of the initial-state energy, and possibly of the initial-state polarization parameters. We will attempt to use polarlued photons to initiate the prosens. Thls ean be done in a number of ways; prantically, tlie problem is being tacked with

a) the photon tagging method,

b) the electroproduction mechanism. In addition, there are plans to use a

ن) backscattered laser photon beam for a bubble chamber experiment proposed at the high-current SIAC. Methods $b$ and $c$ are able to a.tso provido julurization parameters for the ingoing channel, a is not.

A lueutitut, vory recent experiment ${ }^{(59)}$ used the plulun-tuagging scheme at the 7.5 DESY synchrotron, in conjunction with a hydrogen buble chamber, to measure $\gamma p \rightarrow$ hadrono up lo 5 GeV photon energies.

They sbtain a iremostrallung $\gamma$ beam of known energy by having a low-intensity electron beam hit a thin radialor, and momentumanalyzing tho elevtrons which underwent a bremsetrahlung process. A counter hodoscope detects these electrons, covering a range of 0.5 to $5 \mathrm{GeV}$ with a resolution of $\sim 0.1 \mathrm{GeV}$.

The electron beam is swept over the radiator by a pulsed magnet such that the bremsstrahlung photons will hit different sections of the bubble-chamber depending on their time of arrival. Digitizing 
electron momentum, pulsed field, and time-of-arrival, then fully determines the photon energy (if we exclude double-bremsstrahlung processes), so that $\sim 10$ events can be recorded for each 1 msec beam pulse: A careful normalization can be performed by looking at the fully determined events $\gamma p \rightarrow \mathrm{pe}^{+} \mathrm{e}^{-}, \gamma \mathrm{p} \rightarrow \mathrm{p} \pi^{+} \pi^{-}$. We can then find. $\sigma_{\text {tot }}$ simply by counting strong-interaction events.

Fig. 2.8-1 shows the results of the Hamburg group. $\dot{\sigma}_{\text {tot }}$ is seen to decrease from $\sim 200 \mu \mathrm{b}$ at $0.5 \mathrm{GeV}$ to $\sim 110 \mu \mathrm{b}$ from 2 to $5 \mathrm{GeV}$. The errors given in the figure are statistical and systematic. There is good agreement between these numbers and those expected from eq's 2.7-1 and 2.7-2. The figure also shows, for comparison, the single (dotted line) and double (dashed line) $\pi$ production cross sections. The agreement with the VDM predictions ought to be regarded as impressive evidence for its value in describing photon-induced hadronic interactions. The idea of using electro-(or muo-) production to provide kinematical input parameters is presently being employed to determine $\sigma_{\text {tont. }}(\gamma p)$ up to much higher energies, with $e$ and $\mu$ beams at the SLAC, and a $\mu$ beam at the Brookhaven AGS.

The idea is that we can write the double differential crosssection for inelastic electron or muon scattering into solid angle element $d \Omega$ and energy bin $d E^{\prime}$ as $(60)$

$$
\frac{\mathrm{d}^{2} \sigma}{\mathrm{d} \Omega \mathrm{d \textrm {E } ^ { \prime }}} \approx \mathrm{F}\left(\sigma_{\mathrm{T}}+\epsilon \sigma_{\mathrm{S}}\right)
$$

where $F$ and $\epsilon$ arc energy-and angle-dependent kinematical quantities. It can be shows lhat, for small momentum transfer $\mathrm{q}^{2}$, the "scalar" 


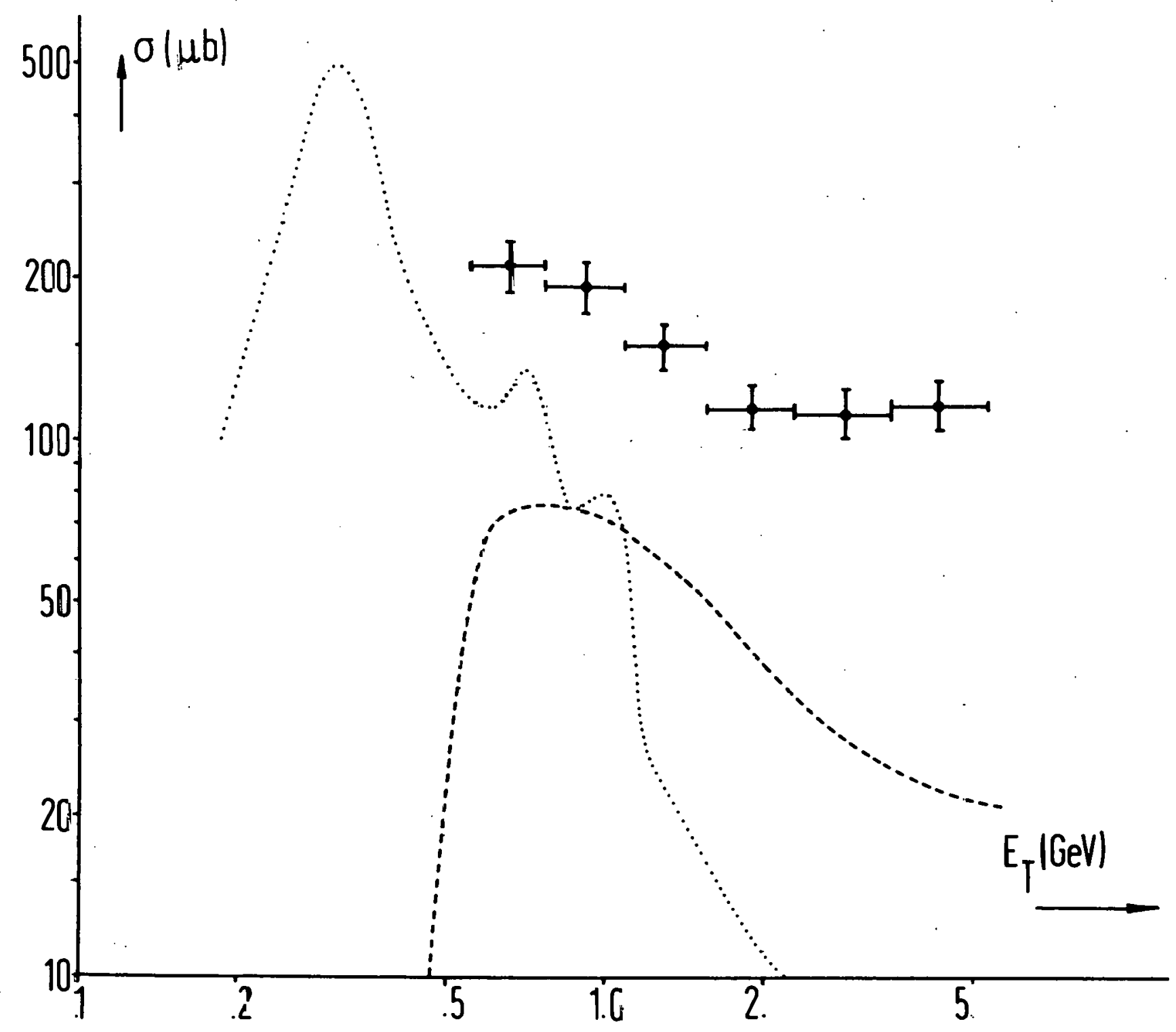

Fig. 2.8-I Measureć. values fer $J_{\text {tot }}\left(\gamma_{\mathrm{p}}\right)$. Dotted curve: single $\pi$ production;

cashed curve: double tot $\pi$ production cross-section. (From Ref.No.59) 
and "transverse" cross-sections $\sigma_{\mathrm{S}}, \sigma_{\mathrm{T}}$ behave like

$$
\begin{aligned}
& \sigma_{S} \underset{q^{2} \rightarrow o}{\longrightarrow} q^{2}\left(\frac{d \sigma}{d q^{2}}\right)_{q^{2}}=0 \\
& \sigma_{T} \underset{q^{2} \rightarrow o}{\longrightarrow} \sigma(\gamma p)
\end{aligned}
$$

Then, if we measure electroproduction at small angles, and plot $I / F \frac{d^{2} \sigma}{d \Omega d E}$ vs. $q^{2}$, we can hope to do a linear extrapolation to $\mathrm{q}^{2}=0$ and find $\sigma_{\text {tot }}(\gamma \mathrm{p})$ in this manner.

The experimental difficulty here lies in the fact that the radiative tail of the elastic scattering mechanism will dominate smallangle behavior and falsify the findings. A compromise has to be found by measuring at values of $\mathrm{q}^{2}$ small enough so that an extrapolation to $q^{2}=$ zero appears credible, but large enough so that the radiative tail (which we believe we can. evaluate well) does not completely drown out the electroproduction process.

This compromise can also be achieved at much smaller angles if we do not take electrons, but.muons as incident particles. The radiative corrections are to be applied with a relative weight $R^{(61)}$

$$
R \approx \frac{\left.l=1 \frac{-a^{2}}{m_{\mu}^{2}}\right)^{-1}}{\ln \left(\frac{-a^{2}}{m_{e}^{2}}\right)^{-1}}
$$

At small momentum transfers $q^{?}$, this gives the $\mu$ experiment a considerable edge over inelastic electron scattering. For example, at 
$\mathrm{q}^{2}=0.02,0.2$, and $1(\mathrm{GeV} / \mathrm{c})^{2}, \mathrm{R} \approx \frac{1}{30}, \frac{1}{6}$, and $\frac{1}{4}$, respectively. Although electron beams have a big advantage through their flux, we will have to have considerable confidence in the calculation of the radiative corrections if we want to do experiments at very small angles. Fortunately, both $\mu$ and e experiments will be performed, and we can look for consistency between them. 
Lecture 3 Photoproduction of Pseudoscalar Mesons at High Energies

3.1 Experimental Data on t-Channel Poles.

In lecture 1, we. focused our attention mainly on s-channel singularities in the photoproduction of pseudoscalars; in the and lecture, after taking a close look at the data, we decided that one particular pole in the t-channel amplitude carried particular importance in the photoproduction of vector mesons. We will now look into the high-energy behavior of the photoproduction amplitudes for pseudoscalars.

We had previously noticed that the bump structure of the total cross section for processes $\gamma p \rightarrow N \pi$ appears to become less and less conspicuous with increasing energy. This is suggestive of the fact that we have to study the $t$ - and $u$-channels and the influence of their singularity structure on the total amplitude; we believe that somewhere between 2 and $3 \mathrm{GeV}$, they will start being the dominant influence.

Let us look at a few relatively well-studied reactions:

a) $\gamma p \rightarrow p \pi^{0}$.

Fig. 3.1-1 shows the forward cross-section measured at DESY ${ }^{(62)}$ and $\mathrm{CEA}{ }^{(63)}$, giving $\frac{d \sigma}{d t}$ at momentum transfers up to $-\mathrm{t}=1.8(\mathrm{GeV} / \mathrm{c})^{2}$. The DESY data were taken with two lead glass Čerenkov counters detecting the $\pi^{0}$ decays into $2 \gamma^{\prime}$ s. so that very small angles were accessible to measurement; the CEA experiment was performed with a spectrometer observing the recoil proton, thereby putting a cut-off on the small-t region (very slow protons cannot be detected in this way). 


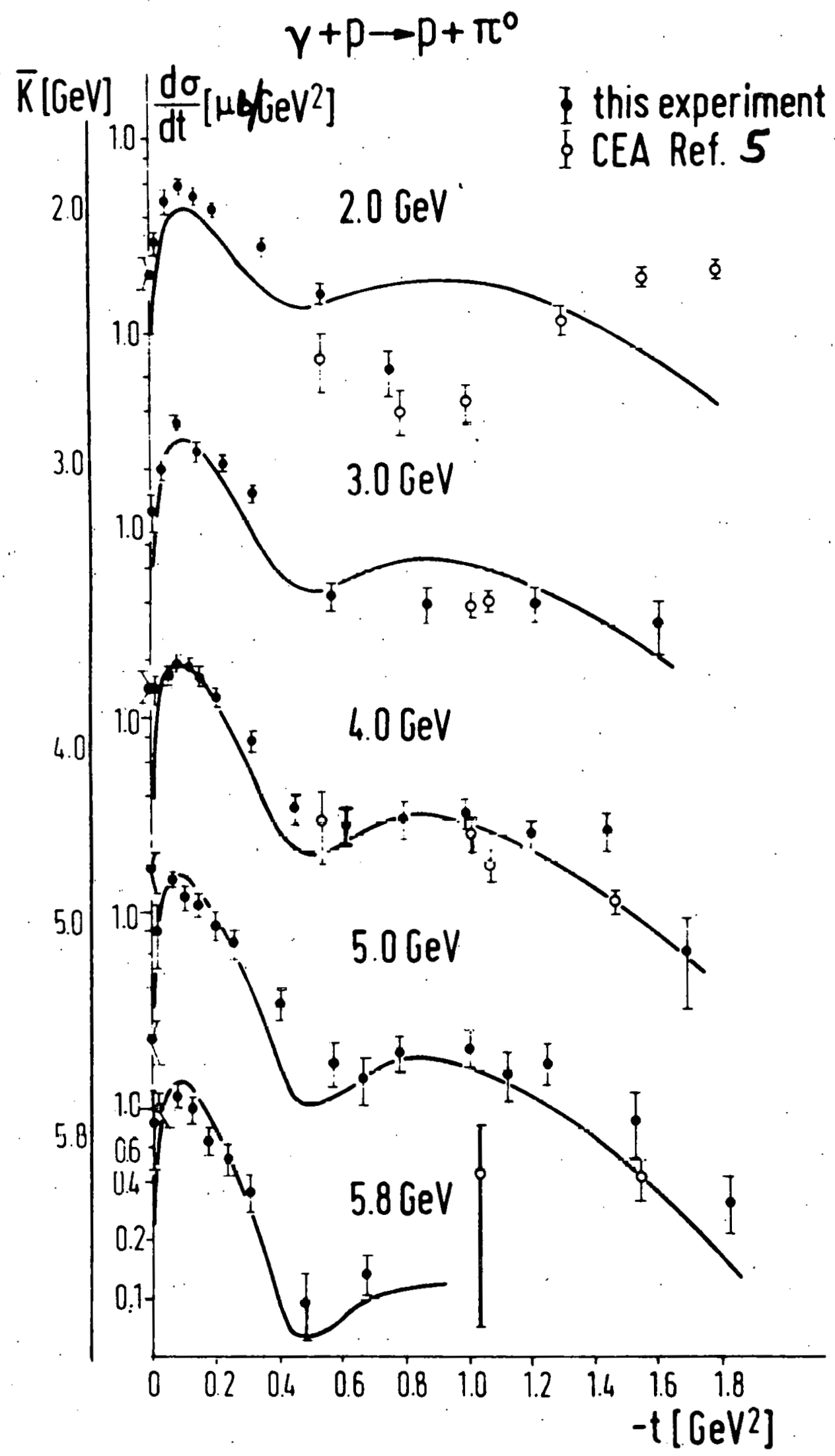

Fig. 3.1-1 Forward differential crose-section for $\gamma \mathrm{p} \rightarrow \mathrm{p} \pi^{\circ}$. (From Ref. No. 62) Fits according to ref. 73. 
We observe the following features: at high energies, 4-6 GeV, there is a very slim forward peak, poorly resolved. At all energies, a dip at moderately small $t$ is followed by a peak at $-t \approx 0.1(\mathrm{GeV} / \mathrm{c})^{2}$; there is a secondary dip somewhere around $-t \approx 0.5(\mathrm{GeV} / \mathrm{c})^{2}$, but its position and its shape are not too constant over the energy range covered:

Fig. 3.1-2 gives a.missing mass curve from the recent one-arm spectrometer experiment performed at SLAC ${ }^{(40)}$ from which we earlier mentioned $\rho^{\circ}$ and $\Phi$ yield data. Due to kinematically forbidden "ghost protons" which show up below $\pi^{\circ}$ threshold, a non-trivial subtraction procedure has to be followed. The accuracy of the $\pi^{0}$ production cross-section given in Fig. 3.1-3 is heavily dependent on this subtraction. The quoted results show nothing of the forward peak and first dip, since again there was a low-energy cut-off on the proton detection, but the cross-section at $6 \mathrm{GeV}$ matches up with the $5.8 \mathrm{GeV}$ data in Fig. 3.1-1. Notice, however, that the secondary dip gets less pronounced and degenerates into a shoulder between 6 and $18 \mathrm{GeV}$.

b) $\gamma p \rightarrow n \pi^{+}$

We have good and precise information on the forward $\pi^{+}$production cross-section from DESY, CEA, and SLAC. It was taken with the aid of single-arm spectrometers (with the large SLAC spectrometer analyzing momenta up to $\sim 20 \mathrm{GeV} / \mathrm{c}$ ); it is tricky to do very-small-angle experiments, so that we have to refer to the literature for details on their realization. 


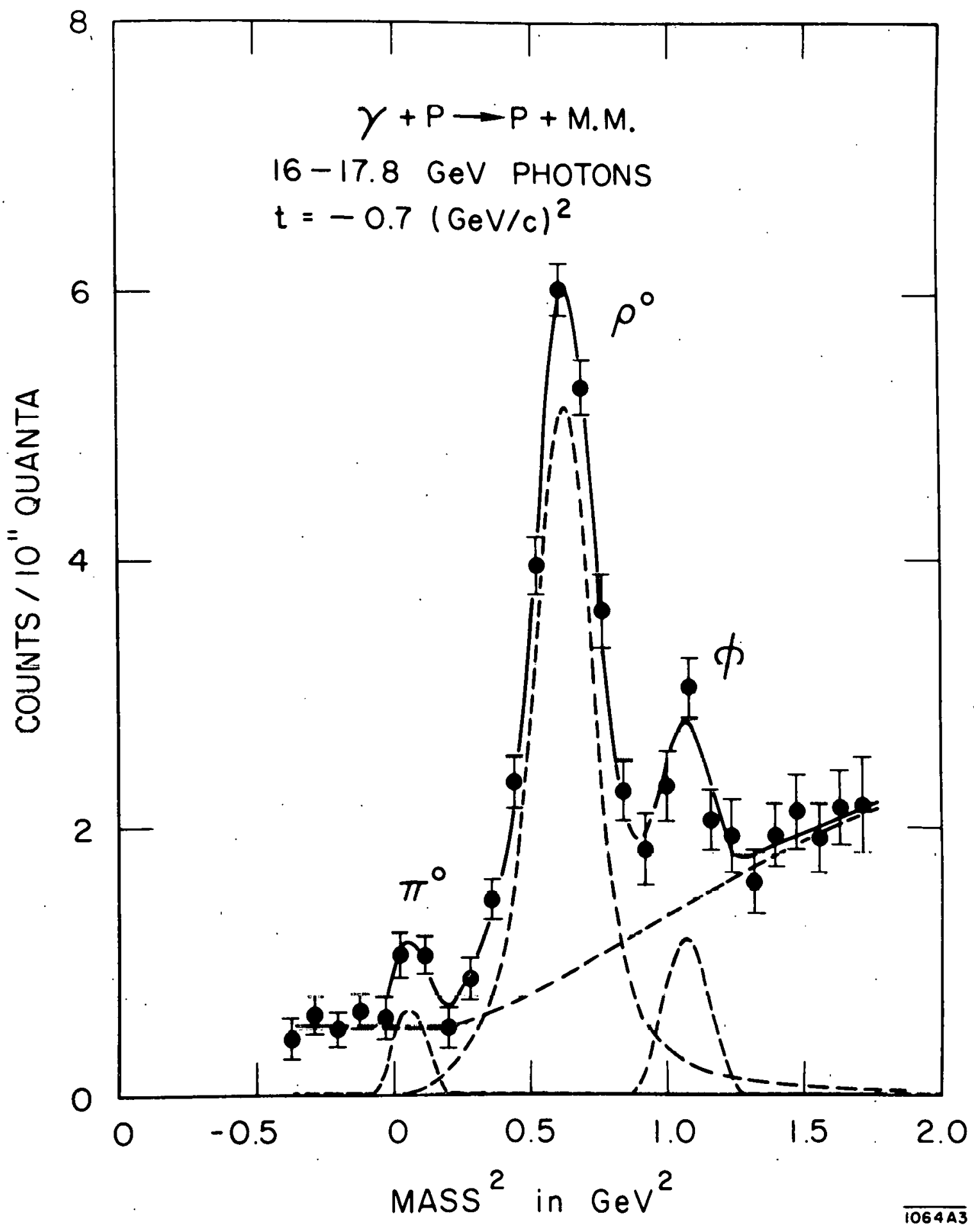

Fig. 3.1-2 Yields of recoil protons due to single boson photoproduction (cf. Fig. 2.2-9). Multiparticle backgrounds have been subtracted out. Best fit curves are indicatec. $\omega$ meson is not resolved. (From Ref. No. 40) 


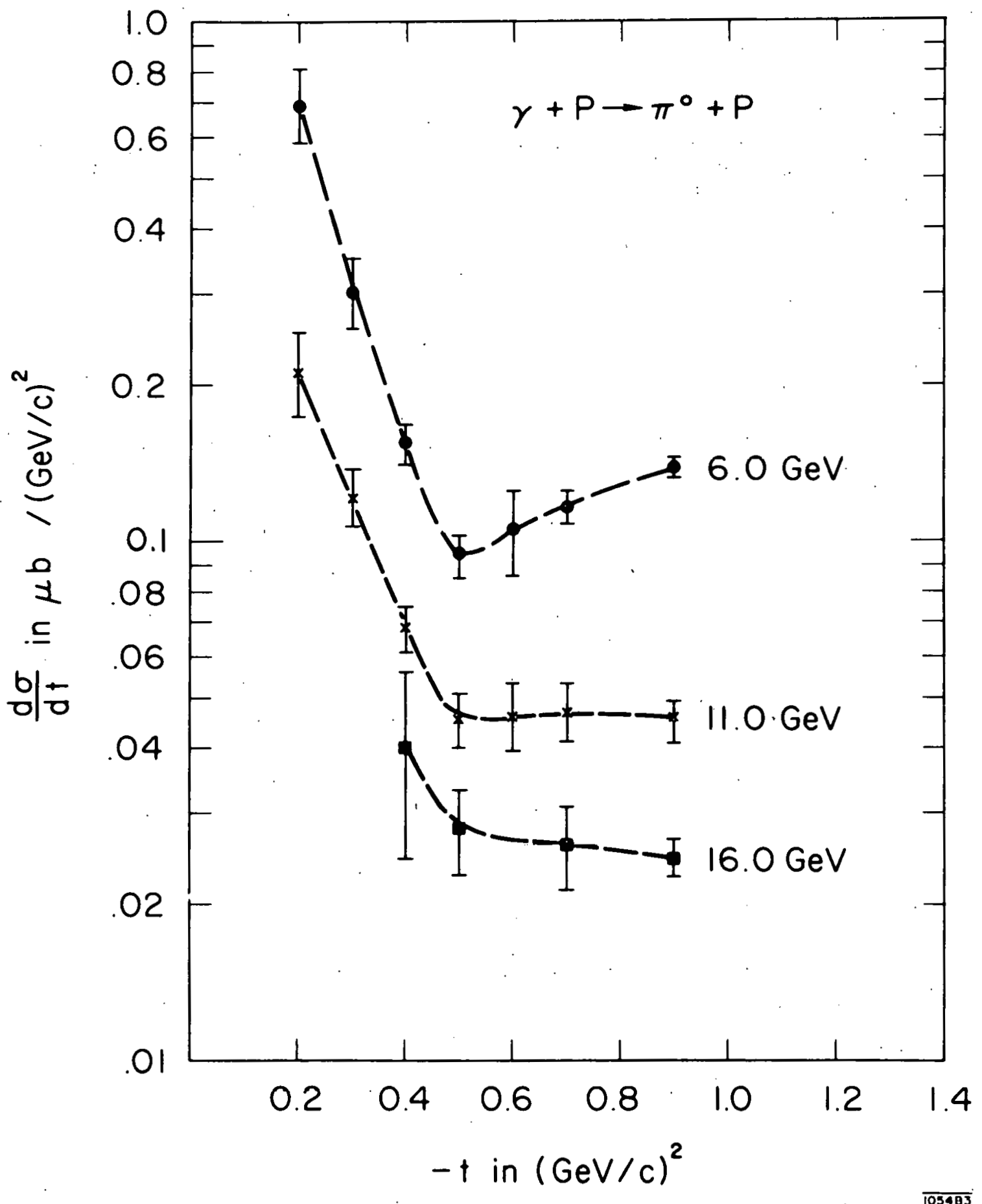

Fig. 3.1-3 High-energy $\pi^{\circ}$ photoproduction: forward differential crose-Gections. (From Ref. No. 40) 
Fig. 3.1-4, summarizes the results of experiments below $1(\mathrm{GeV} / \mathrm{c})^{2}$ momentum transfer $(64,65,66)$. Note the logarithmic scale. The DESY data go down to $-t=10^{-4}(\mathrm{GeV} / \mathrm{c})^{2}$. There is consistent indication for a sharp forward peak, and none for any dip in the region $10^{-4} \leq|t| \leq 1(\mathrm{GeV} / \mathrm{c})^{2}, 2.7 \leq k \leq 5 \mathrm{GeV}$.

'I'he SLAC data, this result and carry it to higher energies and higher momentum transfers (up to $|t|=2(\mathrm{GeV} / \mathrm{c})^{2}$. Moreover, the SLAC data show that outside the sharp forward peak, an exponential behavior describes the data well according to $\frac{d \sigma}{d t} \alpha e^{b t}$, with $b \approx 2$ for energies $k \geq 8 \mathrm{GeV}$ and momentum transfers $|t|$ between 0.07 and 0.6 . At higher $|t|$ values, the exponential fall-off is steeper, with $b \approx 3.3(\mathrm{GeV} / \mathrm{c})^{-2}$. (Fig. 3.1-5).

c) $\gamma \ddot{p} \rightarrow \mathbb{K}^{+} \Lambda$

With the same apparatus as used for the high-energy $\pi^{+}$detection, the Richter group at SLAC took forward photoproduction data on the process $\gamma \mathrm{p} \rightarrow \mathrm{K}^{+} \Lambda$. The resuits, shown in Fig. 3.1-6, are quite dissimilar; there is a distinct dip at small angles, as we would expect from a one-particle exchange mechanism in its simplest formulation (cf. below). There is also, beyond the region of the dip; an exponential fall-off $\frac{d \sigma}{d t} \sim e^{k t}$ with $b \approx 3.1(\mathrm{GeV} / \mathrm{c})^{-2}$ between 5 and $10 \mathrm{GeV}$, close to the $\mathrm{b}$ value used to fit the $\pi^{+}$data.

An obvious cxpcrimental difficulty in this contcxt is the separation of the $K \Lambda$ and $K \Sigma^{\circ}$ final states (cf. Section 1.5). The errors on the $\Sigma^{\circ}$ cross-section are more bothersome than in the $\Lambda$ case 


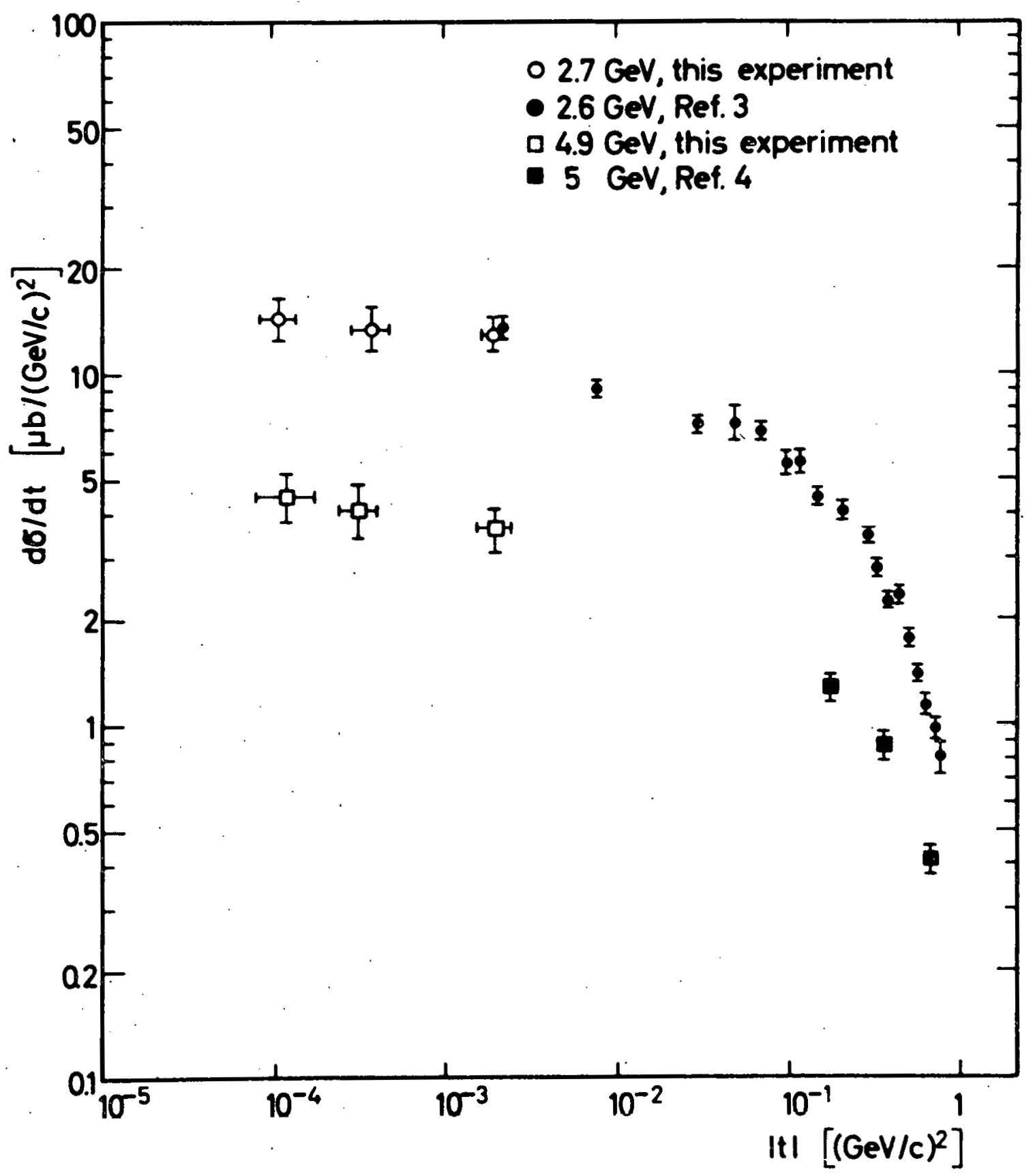

Fig. 3.1-4 Differential cross-section for $\gamma \mathrm{p} \rightarrow \mathrm{n}^{+}$at 2.7 to $5 \mathrm{feV}$. Forward peak, on logar1thmic scale, is indicated down to
$|t| \approx 10^{-4}(\mathrm{GeV} / \mathrm{c})^{2}$. (From Ref. No. 64) 


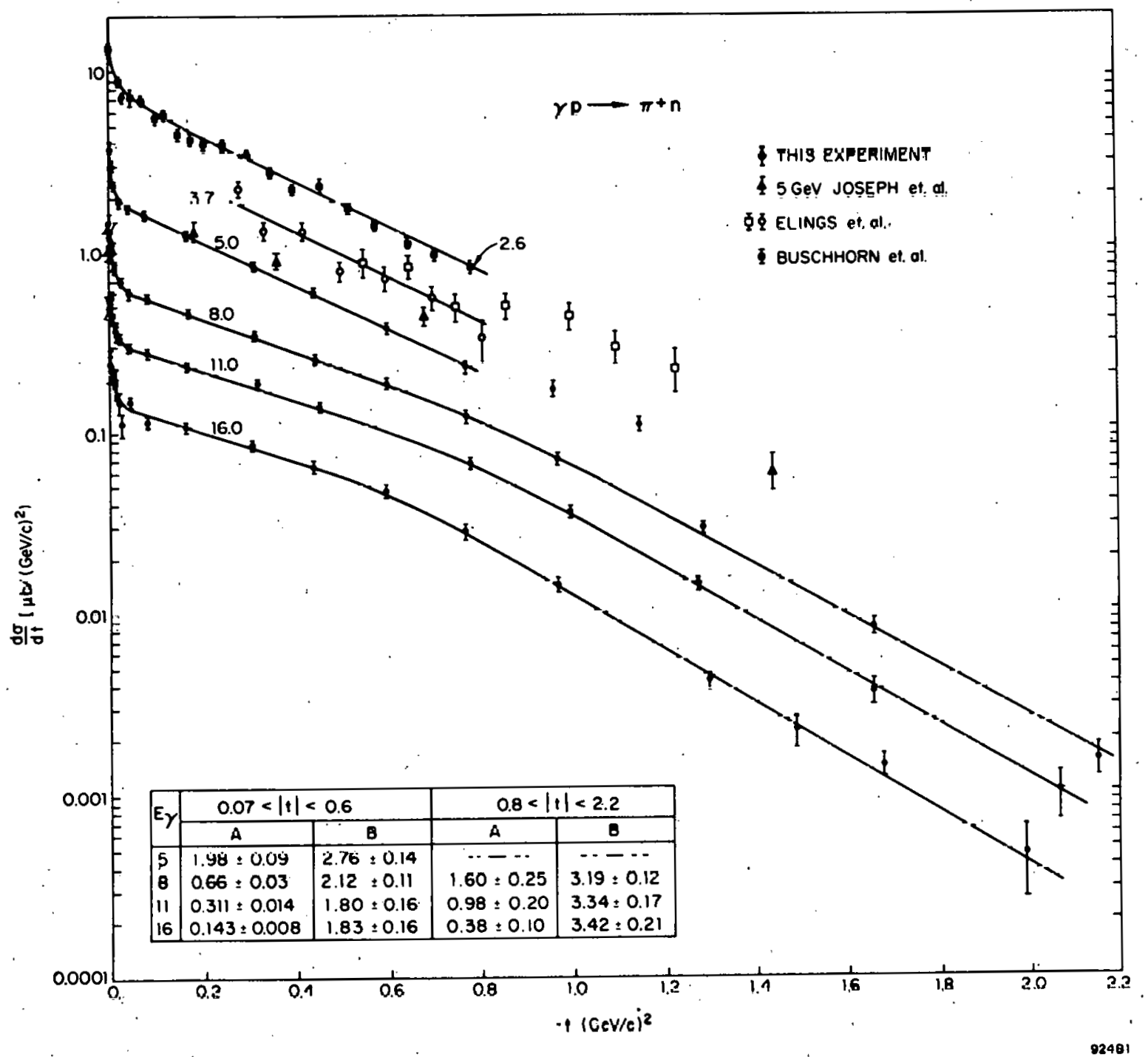

Fig. 3.1-5 High-energy $\pi^{+}$photoproduction. Forward peak and exponential slopes are indicated, with break in slope around $-t=0.7$ $(\mathrm{GeV} / \mathrm{c})^{2}$. (From Ref. No. 67) 


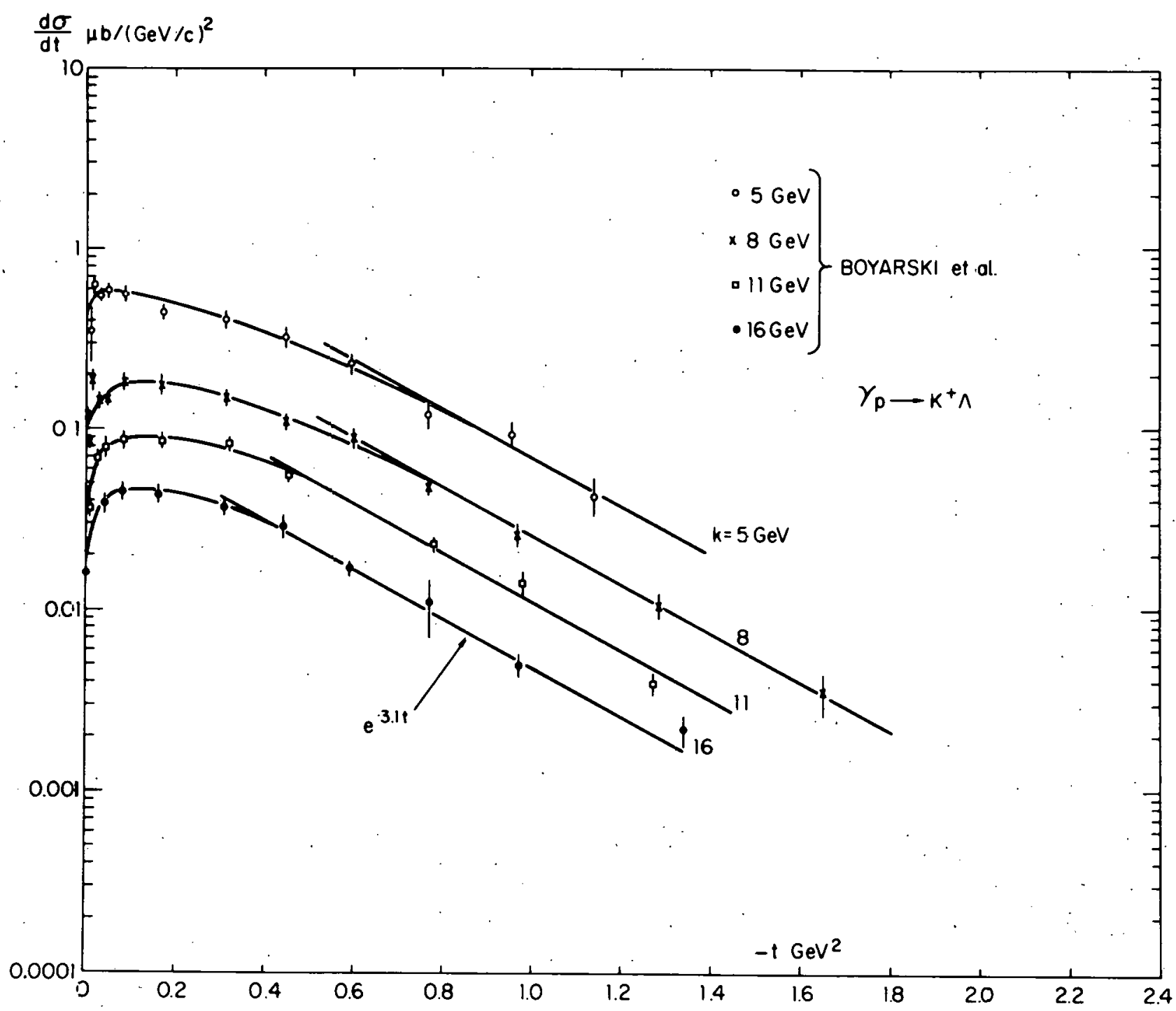

Fig. 3.1-6 High-energy $\mathrm{K}^{+}$photoproduction differential crosssection exhibiting dip at small $|t|$. (From Ref. No.68) 
because of the subtraction necessity. We'Il come back to the $K \Lambda / K \Sigma$ ratio later on (section 3.7 ).

\subsection{Backward Photoproduction of $\pi^{\prime} s$.}

Next, let us look at experimental information on photoproduction processes ịvolving $u$-channel poles; we expect them to largely determine the backward cross-section in the same manner in which t-channel poles are needed to account for forward phenomena. However, let us keep in mind (from section 1.3) that for the backward cross-section, only one of the four helicity amplitudes'will contribute: Therefore, we also expect to see the resonance stmucture in this one amplitude in a very pronounced fáshion, at energies $\leq 3 \mathrm{GeV}$. a) $\gamma \mathrm{p} \rightarrow \mathrm{p} \pi^{\circ}$

Recent DESY data ${ }^{(69)}$ display a pronounced peak-and-dip type structure, when we plul lle $180^{\circ}$ rross-3cction over the photon energy (cf.Fig. 3.2-I). It is seen that mainly the $\Delta$ resonances $(I=3 / C)$ appear to show up appreciably $-\Delta(1920), \Delta(2420)$, and possibly higher mass $\Delta^{\prime} s$. The corresponding peaks in the production cross-section have been used to evaluate the photoexcitation of these states (cf. section 2.6). In terms of the helicity decomposition, the fact that we see these states strongly implies that the amplitude $\mathrm{H}_{4}$ plays a strong role in their excitation, out of initial helicity $1 / 2$ (section 1.3 ).

At higher energies, only very preliminary data from SLAC ${ }^{(70)}$ are presently available. Fig. $3.2-2$ shows the behavior of $\frac{d \sigma}{d u}$.vs. $u$ for four processes, at energies around $10 \mathrm{GeV}$, and momentum transfers 


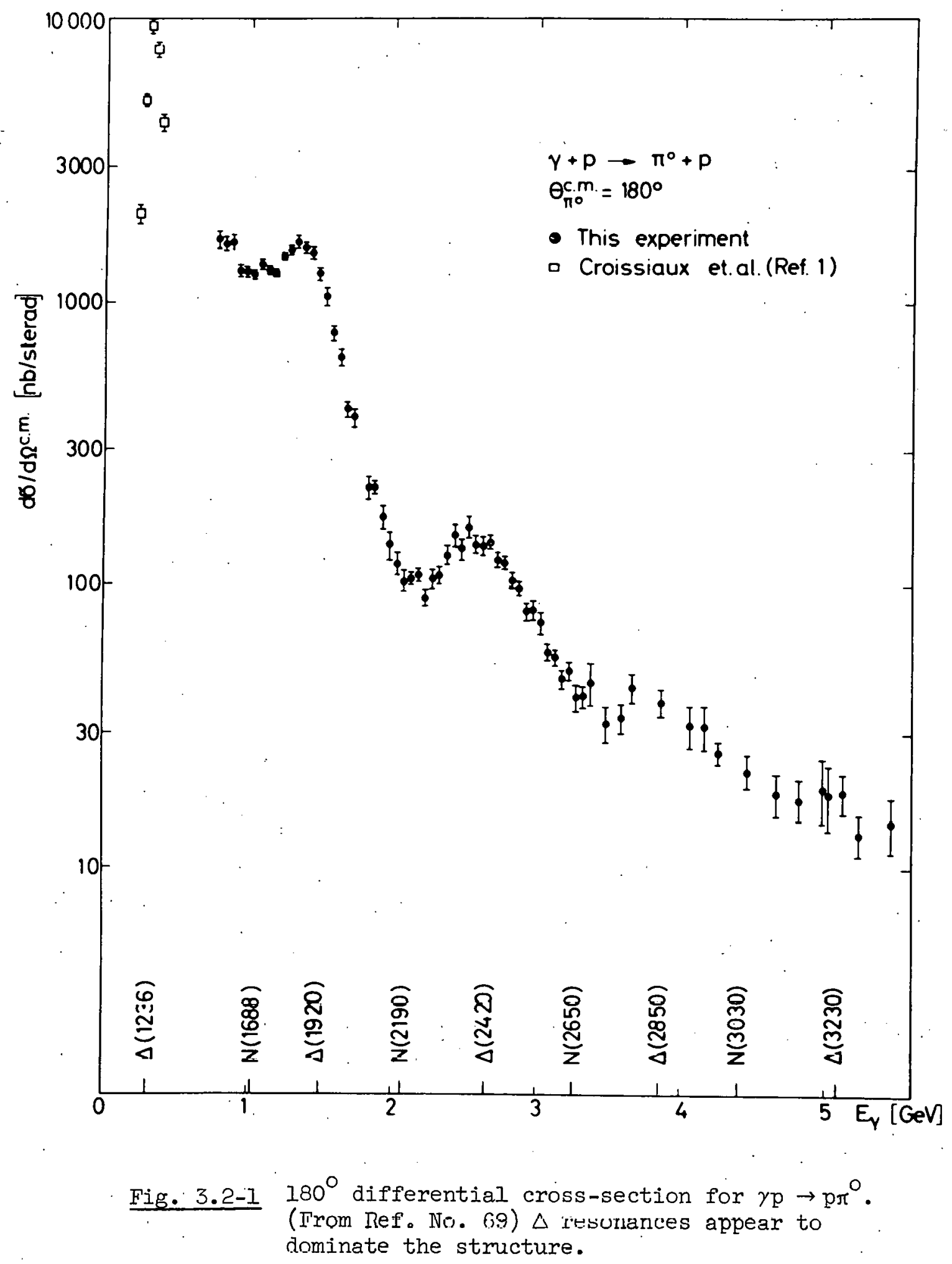




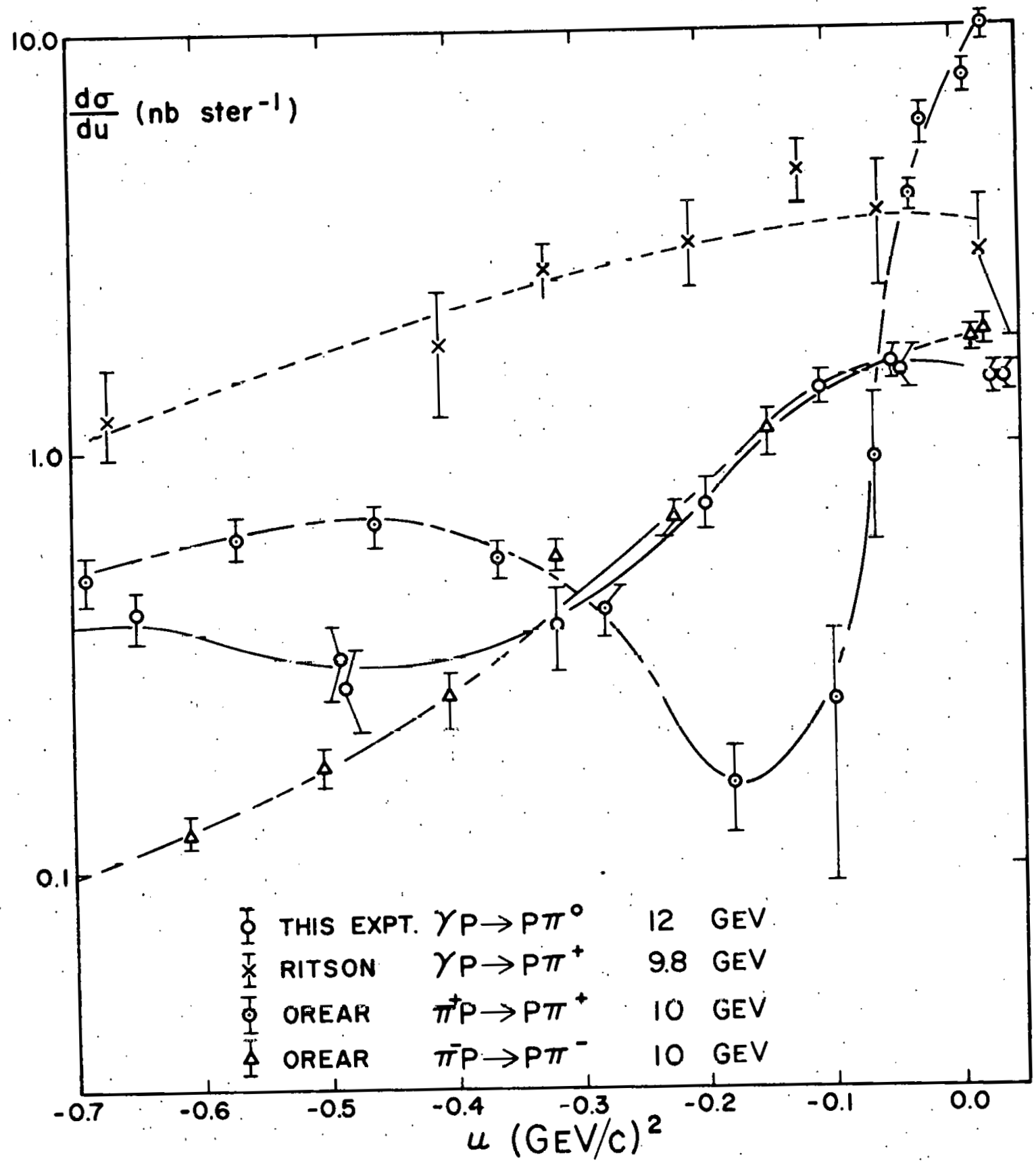

Fig. 3.2-2 Backward differential cross-section $\frac{d \sigma}{d u}(u)$ for $\gamma p \rightarrow p \pi^{\circ}$.
(From Ref. No. 70) Comparison with production and $\pi^{+}$p scattering is indicated. 
-u between 0 and $0.7(\mathrm{GeV} / \mathrm{c})^{2}$ (Note that we now are interested in momentum transfers in the u-channel, for backward production, quite analogously to the $t$ channel analysis in the context of forward production.) The $\pi^{\circ}$ cross-section $\frac{d \sigma}{d u}$ shows no clear structure as a function of $u$ : a slow decrease from $u=0$ down, with a possible flat $\mathrm{dip}$ at $-u \approx 0.5(\mathrm{GeV} / \mathrm{c})^{2}$. For comparison, similar curves are plotted for $\pi^{ \pm} \mathrm{p} \rightarrow \mathrm{p} \pi^{ \pm}$and for $\gamma \mathrm{p} \rightarrow \mathrm{n} \pi^{+}$in the backward direction. Let's just note that the pronounced dip in $\pi^{+} p \rightarrow p \pi^{+}$at $u=-.15(\mathrm{GeV} / \mathrm{c})^{2}$ does not show up in photoproduction.

b) $\gamma \mathrm{p} \rightarrow \mathrm{n} \pi^{+}$

Fig. 3.2-3 gives the angular distribution $\frac{d \sigma}{d u}$ for $\pi^{+}$backward production, at various energies between $2.8 \leq k \leq 9.8 \mathrm{GeV}$. These data were taken at SLAC ${ }^{(71)}$ with the $1.6 \mathrm{GeV} / \mathrm{c}$ spectrometer, again with an excitation-function technique - keeping beam energy and spectrometer angle fixed, and varying the accepted momentum for the spectrometer. As we explained in section 3.1, this method involves a difficult subtraction of backgrounds. At accepted momenta outside the allowed region for photoproduced $\pi^{+}$, there is still a considerable yield due to double processes in the target. In order to believe quantitatively the values given in Fig. 3.2-3, we have to put considerable trust in the subtraction procedure. However, the trend of $\frac{d \sigma}{d u}$ vs. $u$ appears clear - there is a smooth monotonical decrease, without any dip structure. The s-dependence of the backward crosssection is consistent with

$$
\left.\frac{d \sigma}{d u}\right|_{\text {fixed } u} \alpha 3^{-2.9} \pm 0.5
$$




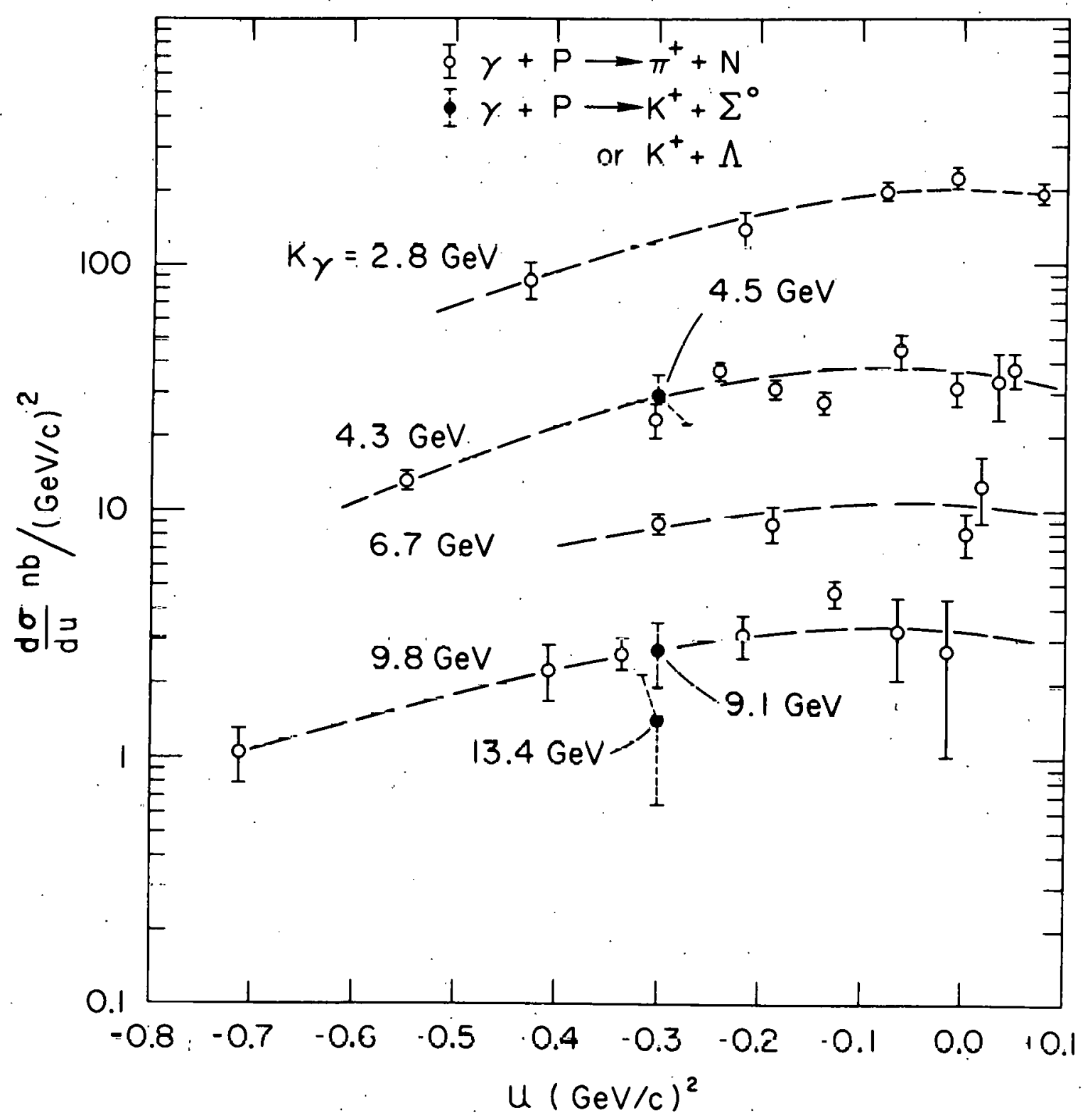

$\overline{\operatorname{indTrit}}$

Fig. 3.2-3 Backward differential cross-section $\frac{d \sigma}{d u}(u)$ for $\gamma \mathrm{p} \rightarrow \mathrm{n} \pi^{+}$. Three points represent same for $\gamma \mathrm{p} \rightarrow \mathrm{K}^{+} \Lambda$ (or $\left.\Sigma^{\circ}\right)$. (From Ref. No. 7I) 
between $k=2.8$ and $13.4 \mathrm{GeV}$. The figure also shows a couple of points for the combined processes $\gamma \mathrm{p} \rightarrow \mathrm{K}^{+} \Lambda$ and $\gamma \mathrm{p} \rightarrow \mathrm{K}^{-1} \Sigma^{\circ}(\Lambda$ and $\Sigma$ could not be experimentally separated), in the backward direction. Since these are the only $\mathrm{K}$ backward data we have, let's just remark that they are roughly as large as the $\pi^{+}$cross-sections at the same energies.

\subsection{The Eimple-Minided Regye Pilture.}

As we try to analyze the data shown in terms of $t-$ and $u-$ channel exchanges, let us recall that in relativistic scattering theory, crossing symmetry links up asymptotic high-energy behavior in the reaction $\mathrm{a} b \rightarrow \mathrm{c} d$ with low-energy poles in the $\mathrm{t}$-channel a $\bar{c} \rightarrow \bar{b}$ and in the $u$-channel a $\bar{d} \rightarrow \bar{b} c$. In other words, the s-channel photoproduction amplitudes at high energies will be governed by. low-energy singularities with the quantum numbers of the $t$ and $u$ channels.

It is well-known that elementary pole diagrams do not yield a successful picture; however, the concept of Regge trajectory exchanges accociatcd with the quanlum rumbers of the corresponding channel has had considerable appeal.

In the simplest case, we have only one trajectory. dominating the forward or backward behavior, e.g., in the parade case $\pi^{-} \mathrm{p} \rightarrow \pi^{\circ} \mathrm{n}$, the $\rho$ exchange diagram, where we now have the entire $\rho$ trajectory (with angular momentum $\alpha_{\rho}(t) \approx 0.6+t$ ) exchanged, and nothing else is expected to contribute. Normally, there will be a spin-flip and a non-flip part of the amplitude, and we can write 


$$
\frac{d \sigma}{d t} \sim|A|^{2} \sim\left(\left|B_{f l i p}(t)\right|^{2}+\left|B_{\text {non-flip }}(t)\right|^{2}\right)\left(\frac{s}{s_{0}}\right)^{2 \alpha(t)-2}
$$

The B's are associated with the residues of the poles which move urith energy in the complex angular momentum plane. Then it becowes clear that for $t=-0.6, \alpha_{\rho}(t=-0.6)=0$, the spin-flip amplitude must have a zero (called a "nonsense zero", since it does not make physical sense that the $\alpha=0$ pole in the $t$ channel could carry across any spin unit.) We expect then, and see, a pronounced dip in the crosssection $\frac{d \sigma}{d t}(t)\left(\pi^{-} p \rightarrow \pi^{o} n\right)$ at $t \approx 0.6$, and we say the dip is due to a nonsense zero in the spin-flip part of the $\rho$ trajectory amplitude. Such dips have been observed in many cases, and we will look out for them in the photoproduction cross-sections - keeping in mind that they refer to the dominance of one trajectory (or at worst, a few) in the ampli.itudes. Let us look at the data presented, and see whether they exhibit characteristics which can be explained in these simple terms.

a) $\gamma \mathrm{p} \rightarrow \mathrm{p} \pi^{\circ}$ Forward

The quantum numbers of the $t$ channel with $C=-1$ suggest the $\omega$ as the leading trajectory. (We know that $\rho \rightarrow \pi \gamma$ and $\Phi \rightarrow \pi \gamma$ are smbll when compared with $\omega \rightarrow \pi \gamma)$ : The simple Regge pole model, with the exchange of the leading trajectory only, would then predict a zero cross-section at $t \approx 0.5(\mathrm{GeV} / \mathrm{c})^{2}$. The lower-energy data show a dip in this region, but not a zero. To remedy the situation, an additional exchange of the $J^{P}=I^{+} B$ meson was suggested $(72,73)$ to fill in the dip, and Fig. 3.1-1 shows the fairly credible fits. 
Some doubts remain, since the decay $B \rightarrow$ I: has never been seen. Above all, the leading trajectory (the $\omega$ ) is expected to dominate the situation more and more with increasing s according to eq. (3.3-1). So, if the B meson trajectory has a slope equal to the $\rho^{\prime} s$, the dip should become more pronounced at higher energies. The recent data at 11 and $16 \mathrm{GeV}$ suggest the opposite (Fig. 3.1-3).

One experimental remedy which can be invoked to study this aspect is the initiation of this process by polarized photons; ${ }^{(74)}$ exchange of a natural parity object $\left(\mathrm{P}=(-1)^{\mathrm{J}}\right)$ will be observed in the production plane only if the photons have a polarization component in the plane. Unnatural parity exchange $\left(P=(-I)^{\mathrm{J}+I}\right)$, like the $I^{+}$ $B$ meson) should be separated out by wleasuring the cross-section in the dip portion, $t \approx-0.5$, with incident photons. polarized perpendicularly to the production plane. Very preliminary results of an experiment to separate $I^{-}$from $I^{+}$exchange in this manner ${ }^{(75)}$ (using a crystal-bremsstrahlung beam for plane polarization) indicate that indeed the $\mathrm{B}\left(\mathrm{I}^{+}\right)$contribution is not what governs behavior in the dip position. For a thorough investigation, we await final results for this and other similar proposed investigations. Another way to explain the peculiar behavior of the $\pi^{\circ}$ crosssection as a function of energy and momentum transfer has been suggested by Ross (76). He proposes that not only the $\omega$ trajectory exchange determines the cross-section, but that a final-state rescatter modifies the picture such that the amplitudes due to

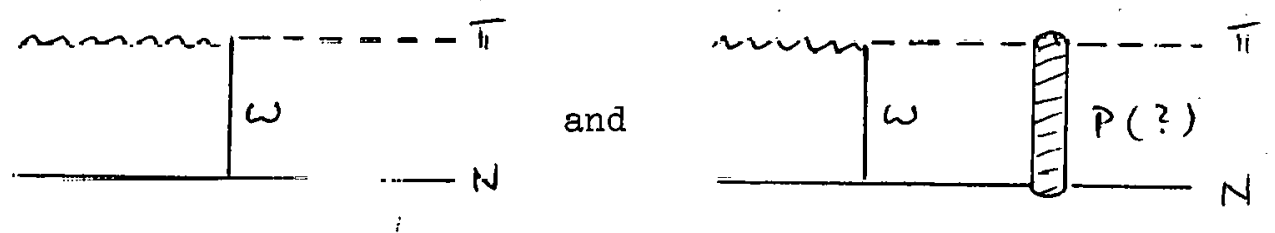


interfere in such a manner that they produce dips - which may then. move and change shape with energy. Similar calculations have been successful in explaining the nucleon polarization in $\pi^{-} p \rightarrow^{0} n$, which was persistently observed experimentally, and due to the $\rho^{\prime} \mathrm{s}$ being the only exchanged trajectory in the reaction, was not expected to occur; this has been, for some time, a particularly embarrassing detail for straight-laced Reggeists.

One can tackle the problem similarly with the expedient of introducing Regge cuts in addition to poles (this is, in a way, whill we did in the case of the above rescatter), or by involving fixed poles in the $J$ plane. We regret that the frame of these lectures does not allow us to spend more time. Rather, we will eagerly wait for more experimental input -- especially from polarized $\gamma$ beams. The overall experimental picture in $\pi^{\circ}$ photoproduction is summarized in Fig. 3.3-1, where the features are shown on a distorted scale; a very narrow peak in the angular distribution due to the "Primakoff graph",

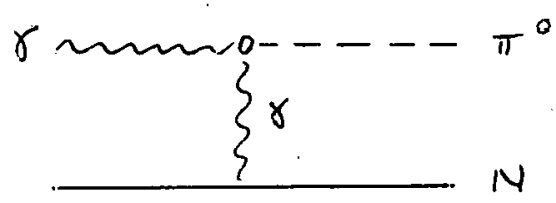

a forward dip, a peak, and secondary dip due to the t-channel $\omega$ pole and its "nonsense zero", and finally a not very steep backward peak 


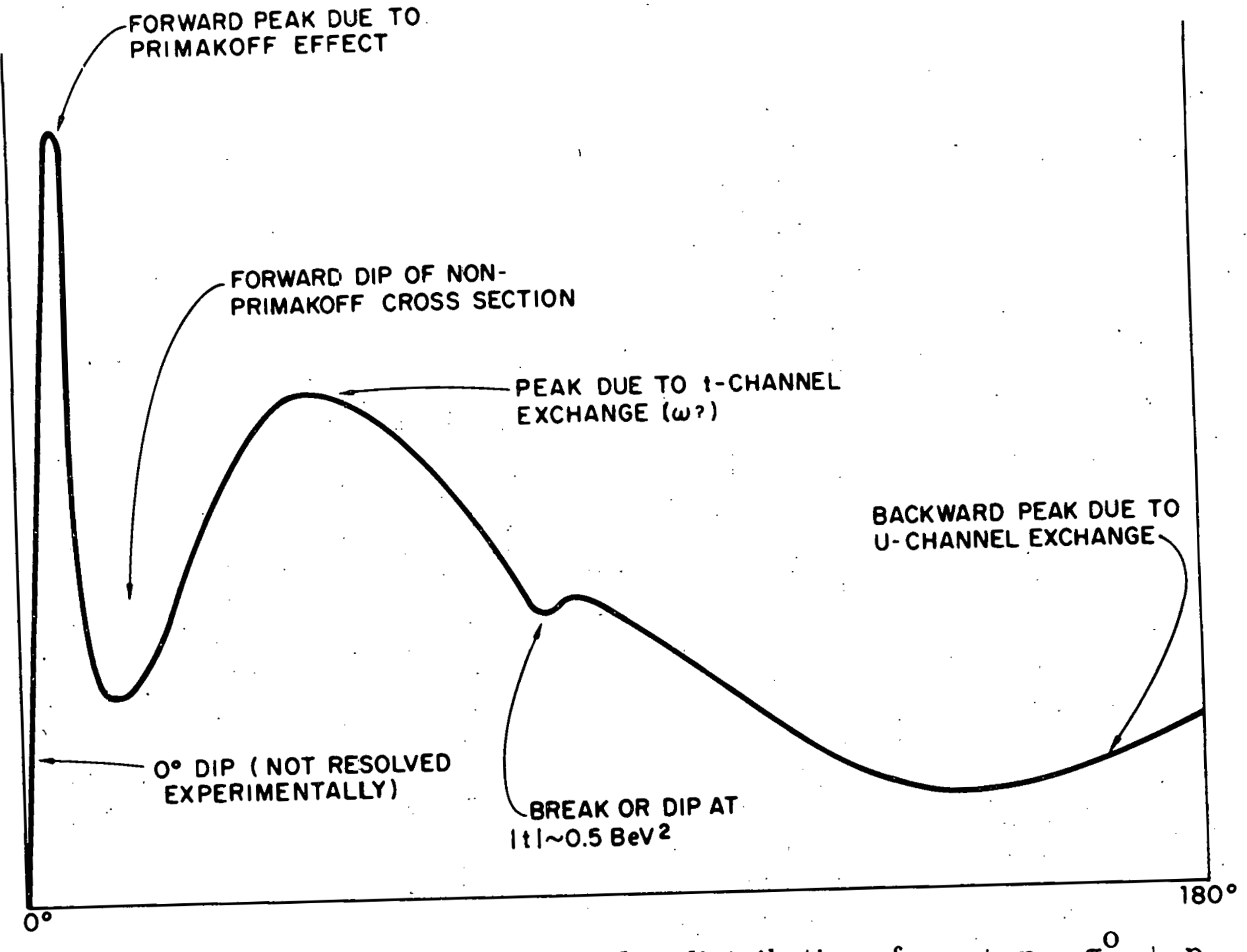

Fig. 3.3-1 General characteristics of angular distributions for $\gamma+p \rightarrow \pi^{0}+p$.

(From Ref. No. 12) 
due to u-channel poles: Fig. $3 \cdot 3-2$ compares the data, on a logarithmic scale, with the simple Regge fit (which does not include the sharp forward peak due to the Primakoff graph). The parameters adopted for the $\omega$ and $B$ trajectories were

$$
\begin{aligned}
& \alpha_{\omega}(t)=0.56+t \\
& \alpha_{B}(t)=-0.30+t
\end{aligned}
$$

b) $\gamma p \rightarrow \pi^{+} n$ forward

There are two features characteristic of the $\pi^{+}$forward data; the sharp peak at $|\mathrm{t}|<0.02(\mathrm{GeV} / \mathrm{c})^{2}$, and the exponential fall-off with $e^{\text {bt }}$ at larger $|t|$ values (Fig. 3.1-5). If we parametrize the latter feature in terms of eq. (3.3-1),

$$
\frac{d \sigma}{d t} \alpha E 2 \alpha(t)-2
$$

we find a trajectory for the leading exchange which is essentially flat with momentum transfer. Fig. 3.3-3 showa that the cnouing values of $\alpha(t)$ are almost compatible with a fixed pole at $\alpha=0$, very different from the trajectory naively expected from the lowestmass t-channel pole.

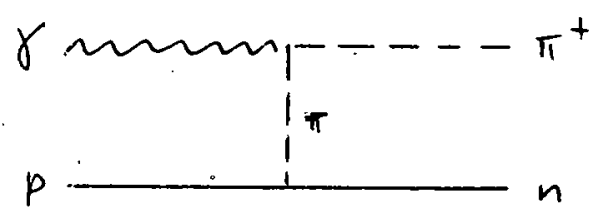

Even if $\alpha(t)$ is presumed to be due to collusion of several trajectories, it remains hard to explain, since, while the unit slope $\pi$ trajectory passes through $\alpha_{\pi}=0$ at $t=0.02(\mathrm{GeV} / \mathrm{c})^{2}$, all other candidates 


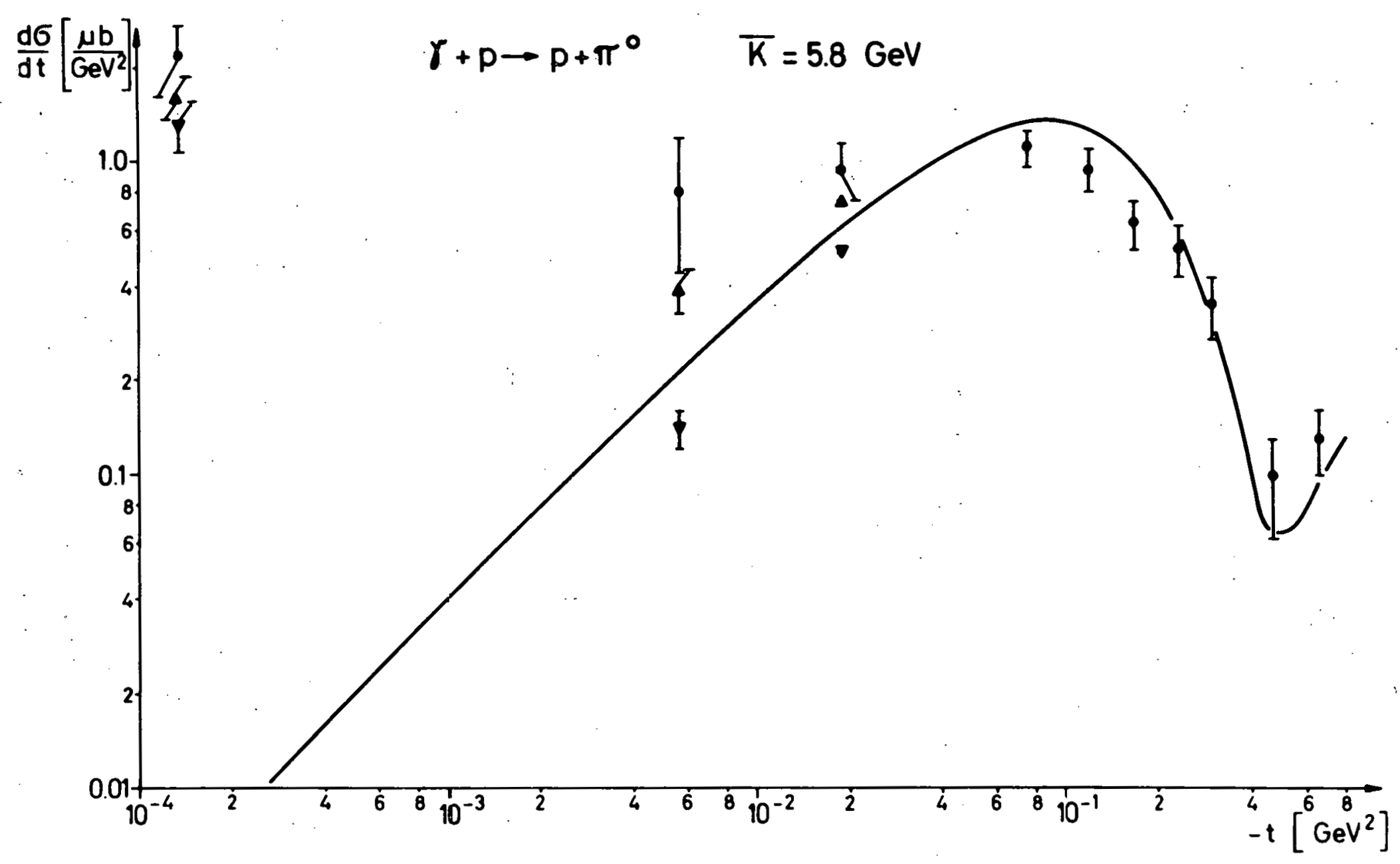

Fig. 3.3-2 Forward $\pi^{\circ}$ photoproduction: fit to data (on exponential scale) using Reggeized $\omega$ and $B$ exchange. (From Ref. No. 62) 


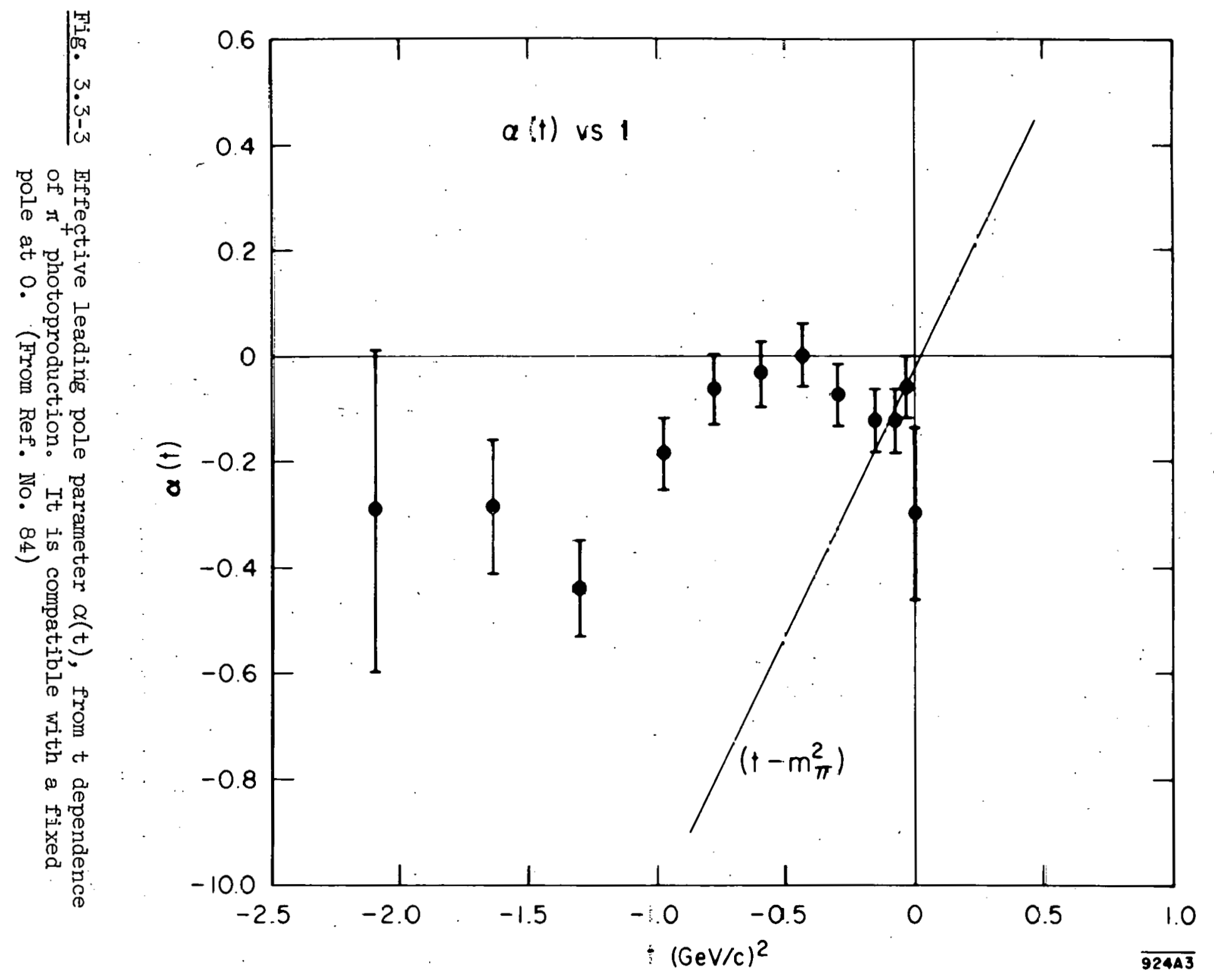


(like the vector mesons) cross through zero at $\sim-0.5(\mathrm{GeV} / \mathrm{c})^{2}$; however, the data indicate that $\alpha(t)$ remains close to zero, out to much larger $t$ values.

We simply note a failure of the simple Regge picture here, and we delay the discussion of the sharp forward peak to section 3.5 ; it cannot be understood in terms of single-trajectory exchange alone.

c) $\gamma \mathrm{p} \rightarrow \mathrm{K}^{+} \Lambda$ Forward

Recall that (cf. Fig. 3.1-6) the $\mathrm{K}^{+}$photoproduction data looked very similar to the $\pi^{+}$results, as long as we do not look at $t<0.02$, where the spectacular difference comes in between forward peak and dip. At $|t|>0.5$, the slope is similar to that in the $\pi^{+}$case, and from this $\frac{d \sigma}{d t} \alpha e^{3.1 t}$ behavior we again deduce a flat effective trajectory. $\alpha_{K}(t) \approx 0$, which we would naively want to associate with the charged $\mathrm{K}$ pole

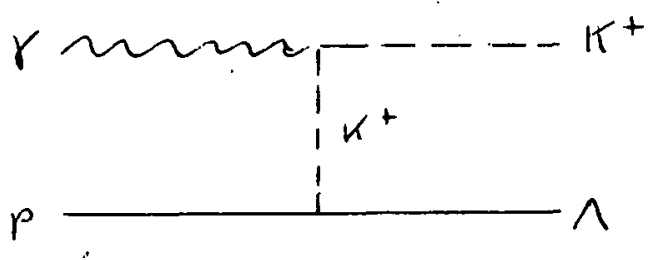

Fig. 3.3-4 shows the resulting trend for $\alpha_{\pi}(t)$, again compatible with a fixed pole at $J=0$, over the entire t-range covered. Again, no interpretation in simple Regge terms appears possible.

d) $\gamma \mathrm{p} \rightarrow \mathrm{p} \pi^{\circ}$ and $\gamma \mathrm{p} \rightarrow \mathrm{n} \pi^{+}$Backward.

Let us look at the vackward cross-sections $\frac{d \sigma}{d u}$ in an equally simpleminded way, trying to explain the data in terms of the exchange of the leading (Iowest-mass) poles. We notice that in elastic tp scattering, 


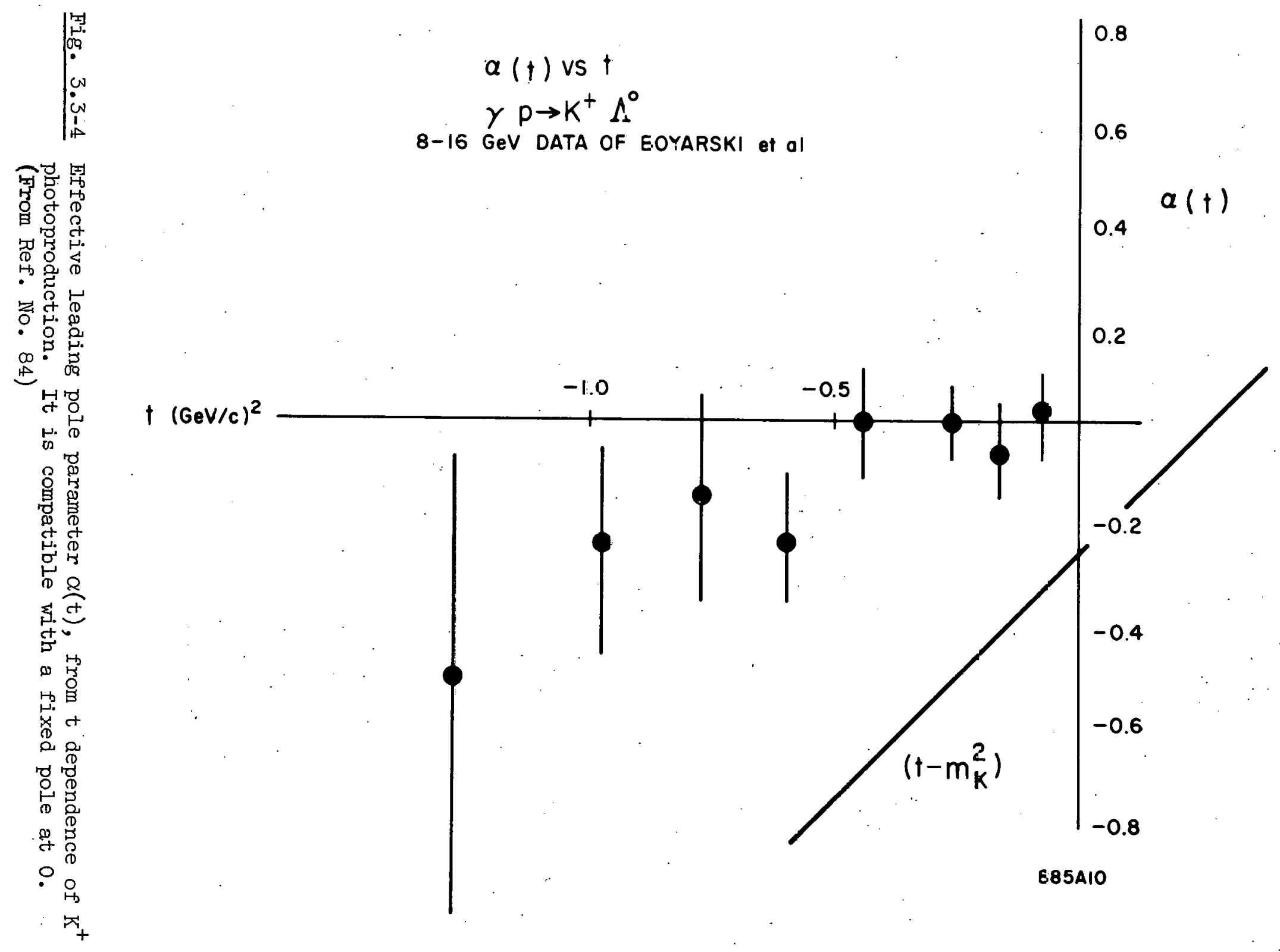


this approach appears successful. Fig. 3.3-5 ${ }^{(77)}$ illustrates our point: For the case $\pi^{+} \mathrm{p} \rightarrow \mathrm{p} \pi^{+}$

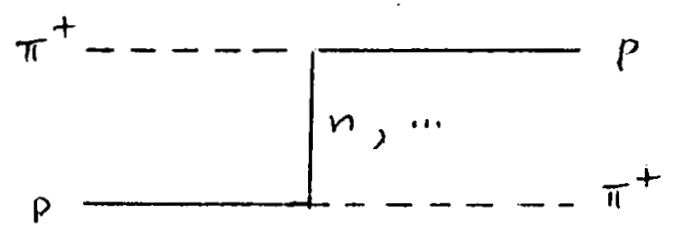

the $t$ channel quantum numbers determine the neutron pole as the lowestmass exchange; the nucleon trajectory, however, has a "nonsense zero" at $\alpha_{N}(u)=I / 2$, corresponding to $-u \approx 0.15(\mathrm{GeV} / \mathrm{c})^{2}$. There is a. distinct dip visible. In the case of $\pi^{-} p$ backward scattering,

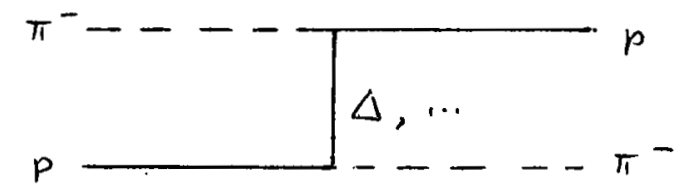

the I spin quantum number of the $t$ channel does not allow for nucleon exchange, but the $\Delta$ trajectory is expected to dominate. This is borne out by the data (also in Fig. 3.3-5): The $\Delta$ trajectory has no nonsense zero in the physical region $u<0$, at $u$ values covered here; it may or may not finally reach the nonseise point at $\alpha_{\Delta}=-3 / 2$, but no dip is visible.

In the photoproduction of $\pi^{\circ}$ and $\pi^{+1}$ in the backward direction, we would expect the diagram

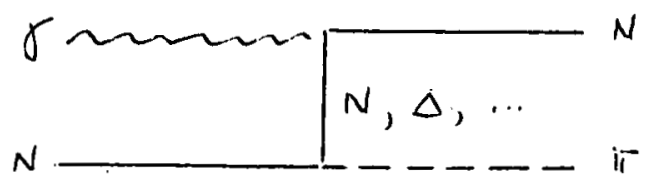

to be dominated by the nucleon pole in the $u$ channel. However, the data (Fig.5 3.2-2 and 3.2-3) make it quite clear that there is no indication of a dip at $-u=0.2$. 

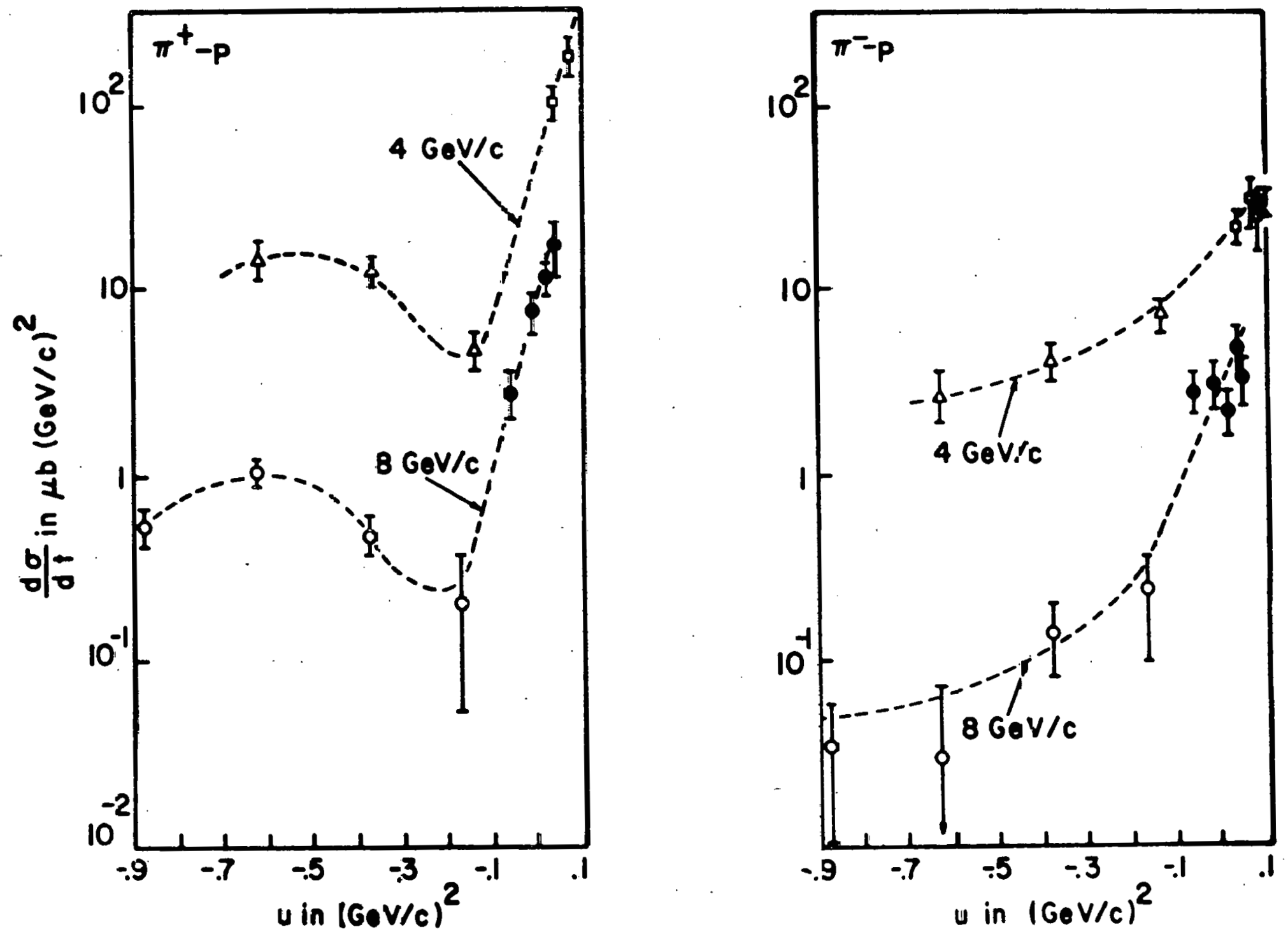

Fig. 3.3-5 Backward differential cross-sections for elastis scattering. $\pi^{ \pm}-p \rightarrow \stackrel{+}{\pi-p}$. (From Ref. No. 77) 
E. A. Paschos ${ }^{(78)}$ at SLAC has recently given a careful evaluation of the backward cross-section for both $\pi p$ elastic scattering and the photoproduction processes. Keeping track carefully of all the kinematical singularities involved, he fits the elastic scattering data with the nucleon $\left(" N{ }_{\alpha}\right.$ ") and $\Delta$ trajectories, respectively. Then, noting that the non-dip at the nucleon nonsense zero in photoproduction makes an interference effect likely, he excludes dominance of nucleon trajectory exchange. Similarly, however, $\Delta$ trajectory exchange is not the only process occurring. For pure $\Delta$ exchange, which has to be isovector, isospin invariance prescribes

$$
\left(\left.\frac{d \sigma}{d u}\right|_{\pi^{0}}=2\left|\frac{d \sigma}{d u}\right|_{\pi^{+}} \text {if } \Delta\right. \text { exchange. }
$$

However, the data make the two cross-sections of eq. (3.3-3) look about equal. Therefore, interference effects between the $\mathbb{N}_{\alpha}$ and the $\Delta$ trajectories are likely to account for the observed structure.

It may not appear too surprising that the coupling of the $\Delta$ to $N_{\gamma}$ is not suppressed with respect to the coupling of the $N_{\alpha}$ trajectory: remember that, for the particularcase of $\pi^{0}$ backward production (Fig. 3.2-1), the $\Delta$ states show up very strongly. Similarly, in the framework of the $\rho$ dominance picture, we recall that the $\Delta^{\prime} s(I=3 / 2)$ are favored in the coupling to the $\rho \mathrm{N}$ system (cf. section 2.1).

3.4. The Not-so-simple Regge Picture: Cuts, Fixed Poles, Conspiracy. In the above treatment, we have looked at the available $\pi$ and $\mathrm{K}$ photoproduction data with the specific question in our minds: Can they 
be reasonably described in terms of the exchange of one or a few leading trajectories, associated with the lowest-mass particles whose quantum numbers can be exchanged in the corresponding channel? The answer appears to be: in the forward direction, certainly not. Neither the sharp peak in $\pi^{+}$production (and the corresponding dip for $\mathrm{K}^{+}$), nor the constancy of ${ }^{\prime \prime} \alpha_{\pi}(t)^{\prime \prime}$ with $t$ for larger $|t|$ values, nor the general behavior of $\pi^{\circ}$ photoproduction are explained in such simple terms.

We cannot, in the context of these lectures, give an account of the theoretical framework of the concepts which may be called to the rescue when the simple picture fails. We'll just mention the main ideas, without even there attempting to be complete.

For one thing, it has consistently been pointed out that the singularity structure of the scattering amplitude in the complex angular momentum plane will normally comprise cuts as well as poles, in full analogy to normal polology in the complex momentum plane. It would be a much-hoped-for accident, if we had only poles, and not cuts, moving in the complex-1 plane.

It is obvious that the presence of cuts would cloud the peakand- dip structure naively expected, and often found, due to dominant poles. Physical concepts leading to such mathematical cuts can be formulated in a number of different ways, one of which is the idea of Ross et al. (76) to postulate rescatters in the ingoing and outgoing channels, in addition to the dominant exchange. Absorption corrections to single exchanges can lead to a similar picture. 
Another way in which cuts can be introduced, but which need not lead to cuts, is through fixed poles in the $\ell$ plane (i.e., poles which do not move as a function of $t$ ). We mentioned the simple Regge analysis of $\pi^{+}$forward production at moderate $t$ values, which led to a picture consistent with the presence of a fixed pole at $J=0$.

There is wide-spread controversy over whether such fixed poles exist in photoproduction $(79,80,81)$. In general, reactions involving spin have possible fixed poles at integer positions of $l$, either positive or negative, $\ell_{p} \leq J_{1}+J_{2}-1$ or $\leq J_{3}+J_{4}-1$, whichever is larger $(82)$. This implies that, in spinless processes, fixed poles are located at negative integer values, so their influence is expected to be masked by the (higher-lying) trajectories of moving poles. I. Jones ${ }^{(83)}$ has pointed out that a fixed pole at $J=0$ will

a) contribute to the "negative signature" amplitude, from which we obtain the above-mentioned nonsense zeroes, such that there is a constant term as $\alpha \rightarrow 0$, filling in the dip; it will not affect the high-energy behavior;

b) will not af'fect the general structure of the "positive signature" amplitude in the vicinity of its nonsense point $\alpha=0$, but rather add a term $\alpha \frac{1}{\mathrm{~s}}$ to the asymptotic value. This will then dominate the crosssection at $\alpha<0$.

At present, there is no compelling evidence which points to the presence of this particular mechanism in photoproduction. One concept which has recently attracted much attention is the 
"conspiracy" of various trajectories - in its most general form the collusion of two or more trajectories to mask kinematical singularities normally occurring in scattering amplitudes. A "conspiracy relation" is then a constraint imposed on the four helicity amplitudes due to the conspiring trajectories at one particular kinematical point, such that the overall amplitude at that point is free from the singularity normally occurring there.

We will specifically mention one case, in which the conspiracy mechanism has been used to explain the absence of a dip in $\pi^{+}$photoproduction at $t=0$, in the next section.

\subsection{Interpretation of Forward Dips or Peaks. Conspiracy or Not? \\ Consider, to start with, the simple one-particle exchange} approach: the only s-channel helicity amplitude which will contribute to the $0^{\circ}$ cross-section will be (cf. section 1.3 ) the so-called flipflip amplitude $A_{\mu}=I / 2, \lambda=I / 2$ ( or $I_{2}$ in our prcvious notation). In this amplitude, angular momentum conservation is effected through simultaneous flip of the nucleon helicity, and a change of helicity between the $\lambda_{k}=1$ photon and the $\pi$. If this double-flip is brought about by the exchange of one particle, its contribution must be. proportional to the momentum transfer $t$, because all helicity flip couplings involve the momentum of the exchanged object at least Iinearly.

In other words, single-particle exchanges lead to $\frac{d \sigma}{d t} \alpha t$ tor small $t$, and since $t=0$ is very close to $0^{\circ}, \frac{d \sigma}{d t}(t=0)=0$ means 
a dip in the forward cross-section.

Now recall the $\pi^{+}$data shown in Fig. 3.1-5; there is a sharp forward peak rather than a dip. How can we explain this?

Richter ${ }^{(84)}$ pointed out that the forward peak can be accounted for in terms of the Born terms alone. The diagrams (cf. section 1.2)

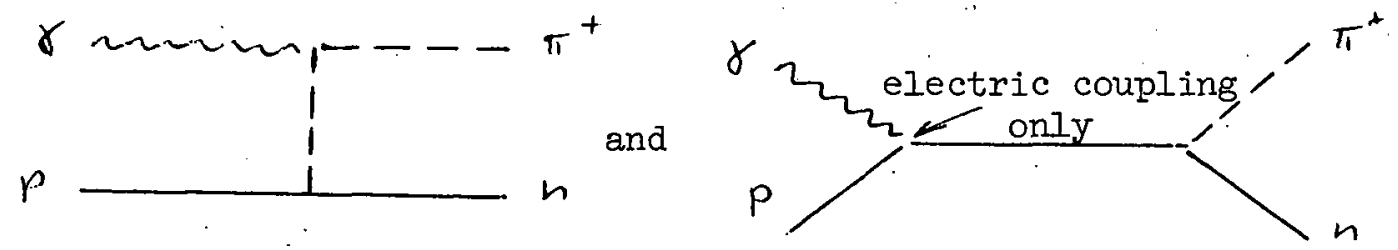

contribute to the forward amplitude like

$$
A \sim \vec{u}(p)\left(\frac{\epsilon \cdot q}{k \cdot q}-\frac{\epsilon \cdot p}{k \cdot p}-\frac{i \sigma}{2 k \cdot p} \epsilon_{k} \mu_{k}\right) u(p)
$$

The first term, the $\pi$ pole in the $t$ channel, dips in the forward direction, as we mentioned. The minimal gauge-invariant form, $\propto$ $\frac{\epsilon \cdot \mathrm{q}}{\mathrm{k} \cdot \mathrm{q}}-\frac{\epsilon \cdot \mathrm{p}}{\mathrm{k} \cdot \mathrm{p}}$, still dips at $\mathrm{t} \rightarrow 0$, but the $\sigma_{\mu \nu}$ term has a $0^{\circ}$ contribution (only in the low partial waves). Fig. 3.5-1 shows that in these terms alone, we can account for the forward behavior at all. energies, whereas at larger momentum tranefere, $|t| \geq m_{\pi}^{2}$, the conventional Regge terms look more appropriate. One might object that at high energies there will certainly be some absorption of the low partial waves, which would eliminate the peak due to the $\sigma_{\mu \nu}$ term. However, absorption would simultaneously affect the first two terms, and they would then no longer dip around $0^{\circ}$, so that the overall picture. remains consistent. 


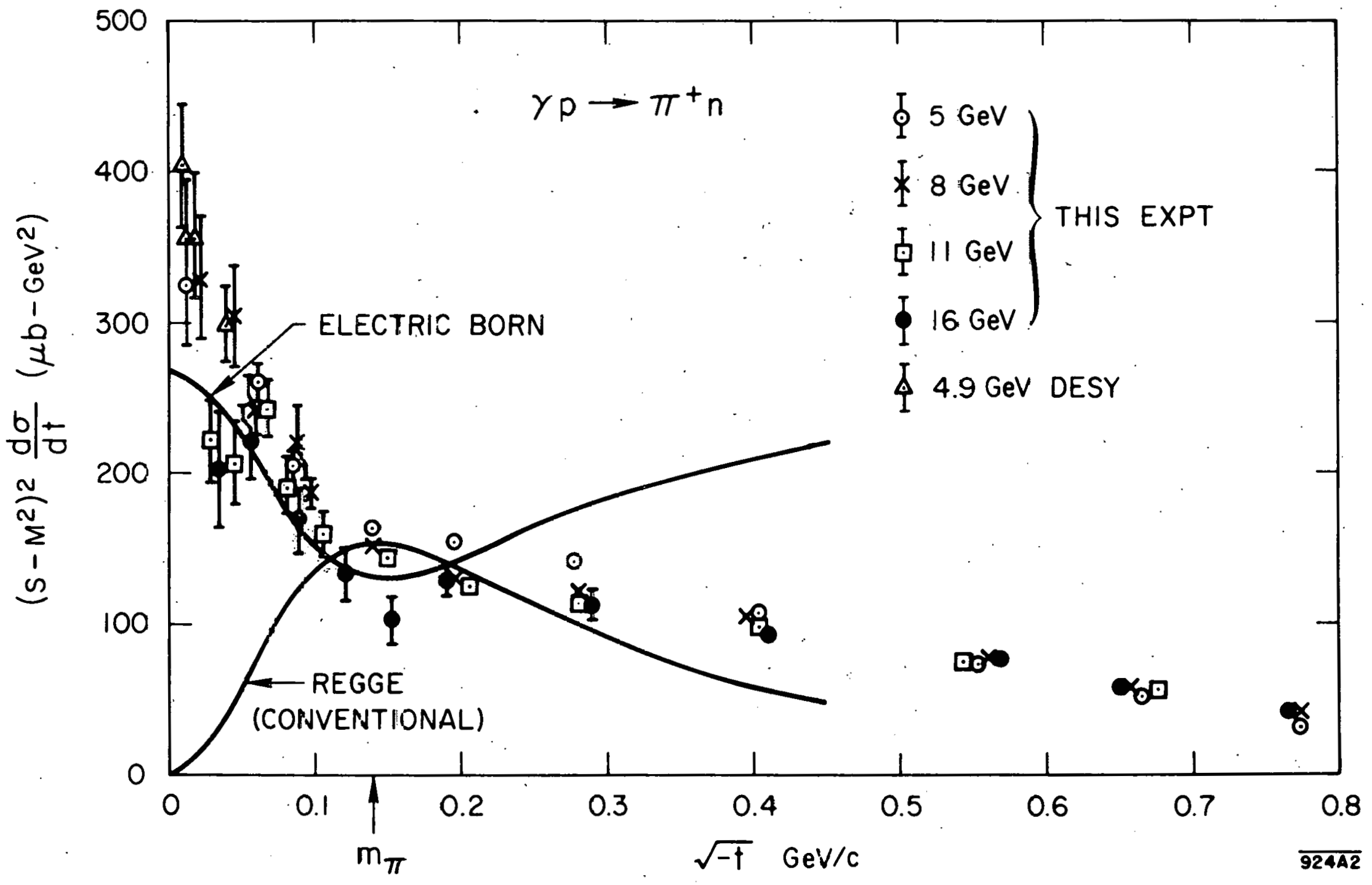

Fig. 3.5-1 Fit to forward peak in $\pi^{+}$photoproduction, using Born terms (eq. 3.5-1) alone. Agreement is seen to be good for $|t| \leq m_{\pi}^{2}$. (From Ref. No. 84) 
The forward peak can also be explained in terms of conspiring trajectories. Ball et a.l. (85) went through the details of $\pi^{+}$and $\mathrm{K}^{+}$ forward production. (For the general formalism, we refer to the literature cited in ref. 85.)

In section 1.3, we wrote down the helicity amplitudes for the s channel. Similarly, it is often convenient to define t-channel helicity amplitudes. We can write them in such a fashion that they are free from kinematical singularities, and find four independent parity-conserving amplitudes, corresponding to the exchange of natural (parity $\left.=(-I)^{l}\right)$ or unnatural $\left(\mathrm{P}^{\prime \prime}=(-I)^{\ell+I}\right.$ ) parity exchanges. Specifically, we can write

$$
\begin{aligned}
& F_{1}, F_{3} \text { contain natural parity exchanges }\left(0^{+}, 1^{-} \ldots\right) \\
& F_{2} \quad \text { contains unnatural parity exchange }\left(0^{-}\right)-- \text {e.g., } \pi \\
& F_{4} \quad \text { contains unnatural parity exchange }\left(1^{+}\right)-- \text {e.g., } A_{1}
\end{aligned}
$$
If we relate these amplitudes at the singular points $t=0,4 m_{\pi}^{2}$ such that the overall amplitude is regular (this is the meaning of the word conspiracy), we get relations among the various $F^{\prime} s$. In particular, the $t_{1}=n$ condition links up $F_{2}$ and $F_{3}$. Thio moans we obtain a condition between the $\pi$ exchange and a normal parity exchange.

We can then postulate that in adiition to the $\pi$ trajectory, whose presence is expected, we also have a conspiring trajectory of opposite parity, the (in-) famous $\pi_{c}\left(0^{+}\right)$trajectory, making up a parity doublet. The specific conspiracy condition then relates the residues $\beta(t)$ and the $\alpha(t)$ values at $t=0$ according to 


$$
\begin{aligned}
& \alpha_{\pi}(0)=\alpha_{\pi_{C}}(0), \\
& \beta_{\pi}(0)=-\frac{m^{2}}{m_{N}} \beta_{\pi_{C}}(0) .
\end{aligned}
$$

With these conditions among the opposite-parity trajectories, the dip at small $|t|$ disappears, and we can actually account for a peak structure. However, in order to have a somewhat palatable picture, we have to postulate also that the conspirator trajectory "choose nonsense" at $\alpha_{\pi_{C}}=0$; otherwice we would have the embarrassing prediction of a $\mathrm{O}^{+}$meson degenerate with the $\pi$, which has somehow. eluded our observation.

After having given this example for a conspiracy-type explanation of the forward peak, we have to add that this approach does contain some uneasy features. In particular, we have to make the residue function $\beta_{\pi}(t)$ of the $\pi$ trajectory vary strongly with $t$ in order to get a consistent picture, whereas we leave the conspirator residue constant. Also, for a simultaneous explanation of the dip in forward $\mathrm{K}^{+}$production, the presence of a conspirator $\mathrm{K}_{\mathrm{C}}$ will have to be suppressed in its influence on the $0^{\circ}$ amplitude. This may be explained by the closeness in mass of $K$ and $K^{*}$ (much closer than $\pi$ and $\rho$ ), so that vector meson exchange may bring about the overall dip in the $\mathrm{K}^{+}$ cross-section.

Amati et al. (86) shunned these features and showed that a slowly t-dependent background interferring with the $\pi$ pole may also be used to explain the forward data. It may be interpreted as steming from a fixed pole, or from absorptive corrections to the $\pi$ pole. 


\subsection{The $\pi^{-} / \pi^{+}$Ratio}

A very important addition to our understanding of the $\pi$ photoproduction process can be expected from the study of the ratio $R$ defined as

$$
\mathrm{R} \equiv \frac{\gamma \mathrm{d} \rightarrow \pi^{-} \mathrm{p}(\mathrm{p})}{\gamma \mathrm{d} \rightarrow \pi^{+} \mathrm{n}(\mathrm{n})}
$$

where the (p), (n) are supposed to be "spectator" particles, not directly involved in the reactions. We have experimental results from CEA ${ }^{(87)}$ and DESY ${ }^{(88)}$, and expect more from SLAC at higher energies.

The analysis of such data is meaningful only if we make sure that the spectator model is solid in the momentum transfer range covered. The earlier experiment ${ }^{(87)}$ showed (Fig. 3.6-1) a ratio $\mathrm{R}$ which is strongly momentum-transfer dependent, and is considerably smaller than unity at the lowest $t$-value measured, $-t=0.4(\mathrm{GeV} / \mathrm{c})^{2}$. This value for $R$ may be taken as an indication for considerable interference between (in terms of the vector dominance model) the diagrams
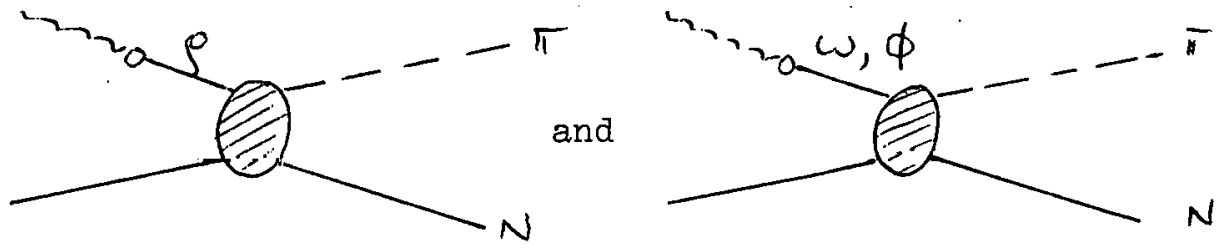

Although $\omega$ and $\Phi$ are more weakly coupled to the photon (cf. section 2.5) than the $\rho$, the fact that $\omega$ and $\rho$ interfere with different signs in $\pi^{+}$and $\pi^{-}$production may well account for this ratio (see eq. 2.6-4). The more recent Hamburg experiment ${ }^{(88)}$ investigated the ratio $R$ down to much smaller $|t|$ values. Fig. $(3.6-2)$ shows results of $\pi^{+}$ and $\pi^{-}$cross-sections from deuterium, and $\pi^{+}$from hydrogen. It is seen 


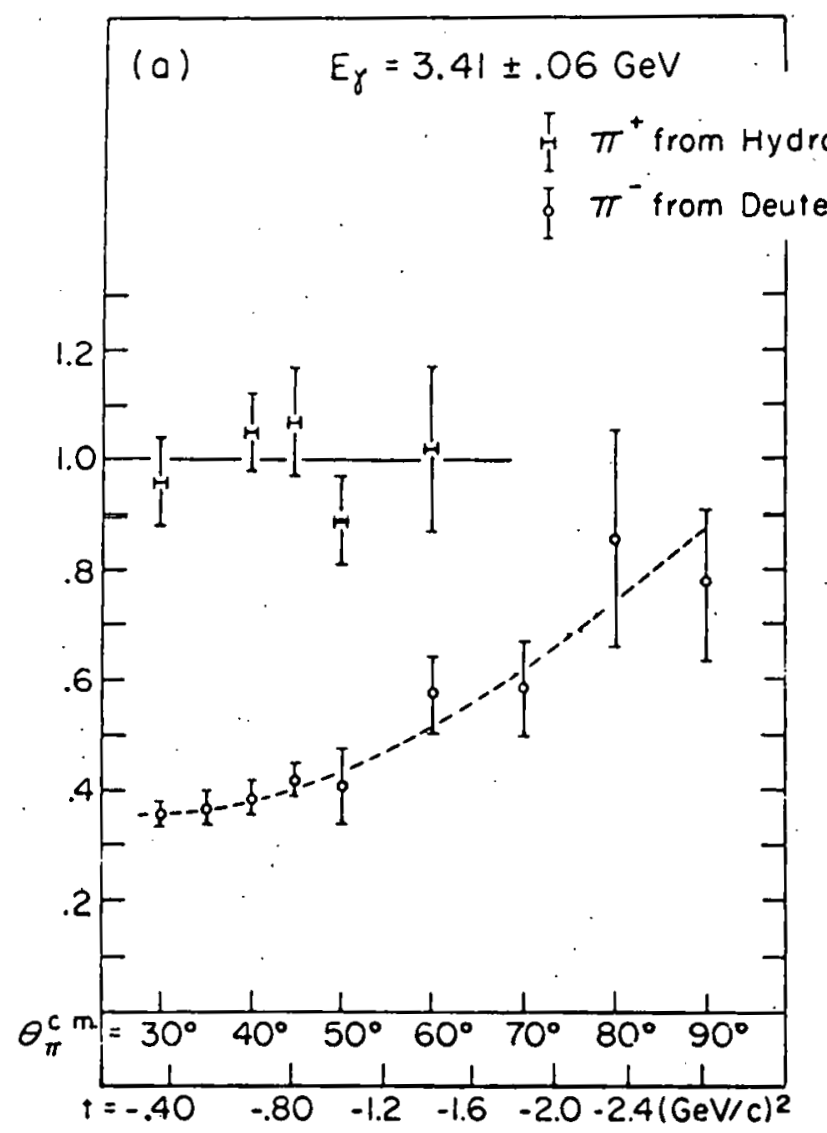

(b) $\quad \theta_{\pi}^{\text {c.m. }}=45^{\circ}$

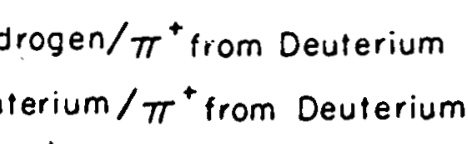




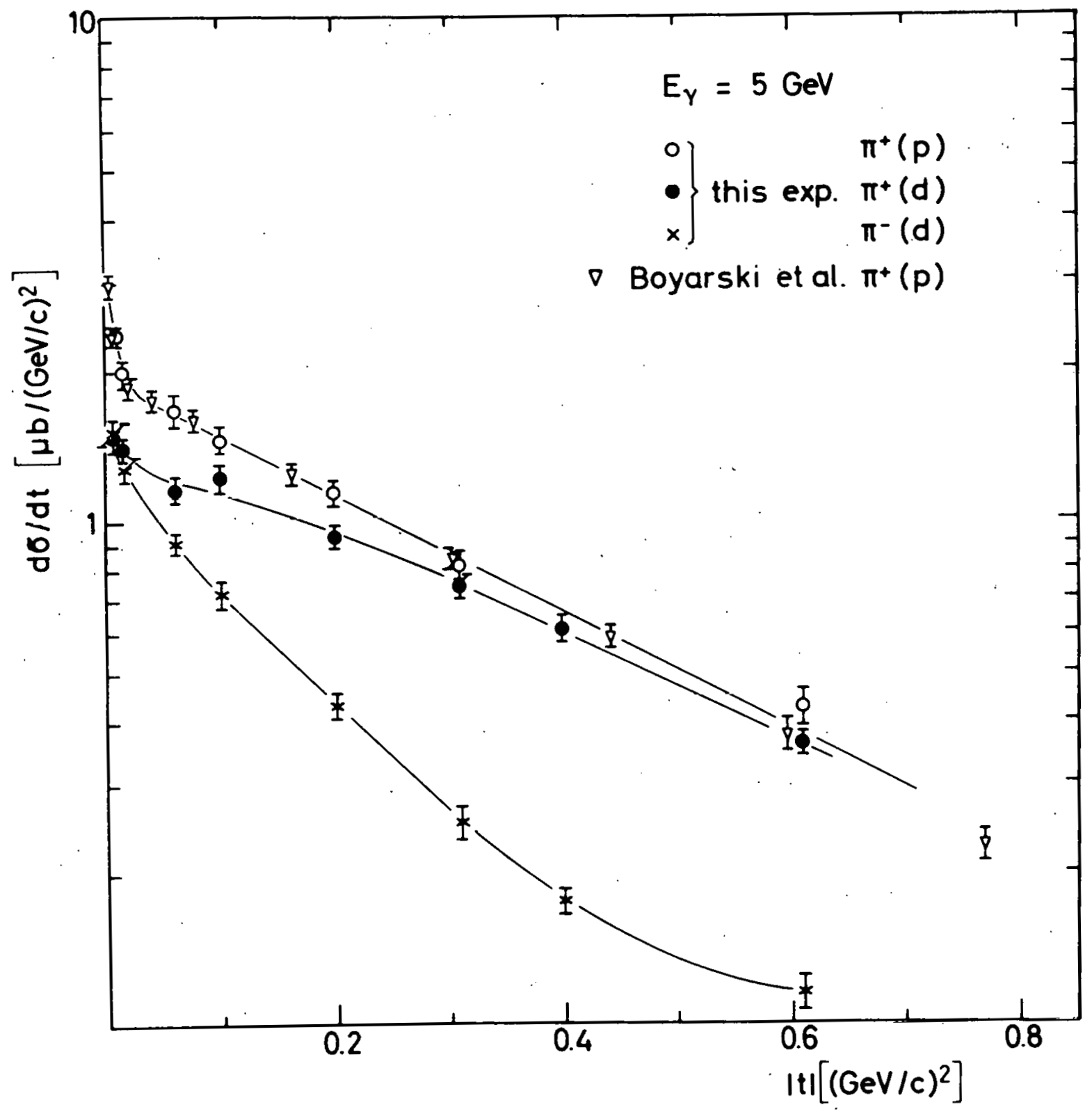

Fig. 3.6-2 Differential cross-sections for $\pi^{+}$photoproduction off hydrogen and deuterium; and for $\pi^{-}$photoproduction off deilterium. (From Ref. No. 88) 
that at small $t$, the $\pi^{+}$rates off hydrogen are significantly larger than those off deuterium. This fact immediately points up one necessary correction to the spectator model; it is due to the Pauli principle which will not permit two final-state neutrons in the same spin and energy state. At larger $|t|$ values, this difference disappears.

There are more corrections we have to apply if we want to extract the free neutron (or free proton) cross-sections from experiments on deuterium. There are "Glauber corrections" for multiple nuclear processes, and the effects of Fermi motion of the nucleons within the deuterium nucleus. However, all of these corrections are expected to be closely similar for both reactions occurring in eq. $3.6-1$, so that the ratio $R$ is not affected. If we want to find the free neutron cross section $\frac{d \sigma}{d t}\left(\gamma \mathrm{n} \rightarrow \mathrm{p} \pi^{-}\right)$trom $\frac{d \sigma}{d t}\left(\gamma d \rightarrow \mathrm{pp} \pi^{-}\right)$, we can take the corrections empirically from the corresponding $\pi^{+}$cross-sections as shown in the two upper curves of Fig. 3.6-2.

The ratio $R$ is plotted, in Fig. 3.6-3, for $0.005 \leq|t| \leq 0.8$ $(\mathrm{GeV} / \mathrm{c})^{2}$. While at $-t=0.4$ the previous ratio is confirmed, the ratio appears to be unity in the very forward direction.

In the spirit of the previous two sections, the $\pi^{-} / \pi^{+}$ratio in the region of the forward peak may provide a crucial test of some of the models proposed for the non-dip structure. In terms of an exchange model, either elementary or Regge-ized, $R \neq I$ implies an interference between exchanged systems of different G-parity. $R(t=0) \approx 1$, as experimentally found, is therefore compatible with the assumption of 


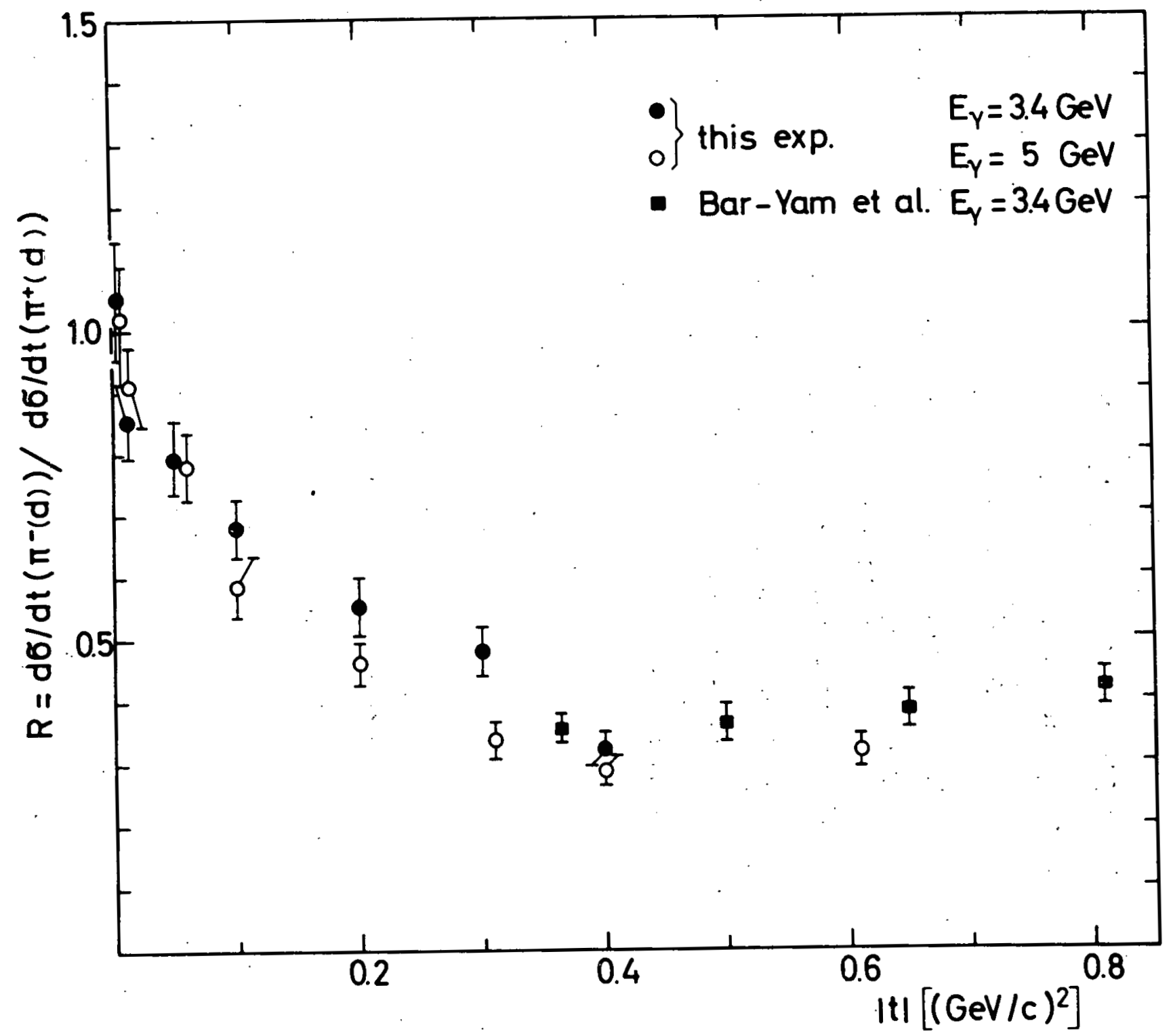

Fig. 3.6-3 Momentum-transfer dependence of the $\pi^{-} / \pi^{+}$ratio R. (From Ref. No. 88) 
conspiring $\pi$ and $\pi_{C}\left(0^{+}\right)$trajectories, since they are of the same G-parity. It will be interesting to extend these measurements to higher energies.

For consistency, we show in Fig. 3.6-4 the similarity of the energy dependence of the differential cross-section for $\pi^{+}$and $\pi^{-}$ production at $\left(t=-0.1(\mathrm{GeV} / \mathrm{c})^{2}\right.$. However, we should point out that the momentum transfer dependence of the differential cross-sections $\frac{d \sigma}{d t}\left(\gamma p \rightarrow n \pi^{+}\right)$and $\frac{d u}{d t}\left(\gamma n \rightarrow p \pi^{-}\right)$looks quite dissimilar; the two low curves in Fig. 3.6-2 point up this difference for $\pi^{+}$and $\pi^{-}$production off deuterium, as reflected in the $t$ dependence of $R$. We can use our arguments about the similarity of the necessary corrections to find the free neutron cross-section through the relation

$$
\frac{d \sigma}{d t}\left(\gamma n \rightarrow \mu^{-}\right)=R \frac{d \sigma}{d t}\left(\gamma p \rightarrow n \pi^{+}\right)
$$

Its $t$-dependence is obviously very different from the $\pi^{+}$production process; the forward spike is not affected, but the $\pi^{-}$cross-section continues to fall of f' more rapidly with increasing $|\mathrm{t}|$. We can formulate this in terms of the amplitudes induced by isoscalar and isovector photons: the squared isovector and isoscalar amplitudes

$$
\left|A_{s}\right|^{2}+\left|A_{v}\right|^{2}=\frac{(I+R)}{\varepsilon} \frac{d \sigma}{d t}\left(\gamma p \rightarrow n \pi^{+}\right)
$$

lead to the forward peaks, whereas the interference term

$$
2 A_{S} A_{v}=\frac{(I-R)}{2} \frac{d \sigma}{d t}\left(\gamma p \rightarrow n \pi^{+}\right)
$$

is absent in the forward direction, since, for very small $|t|, R \approx 1$. 


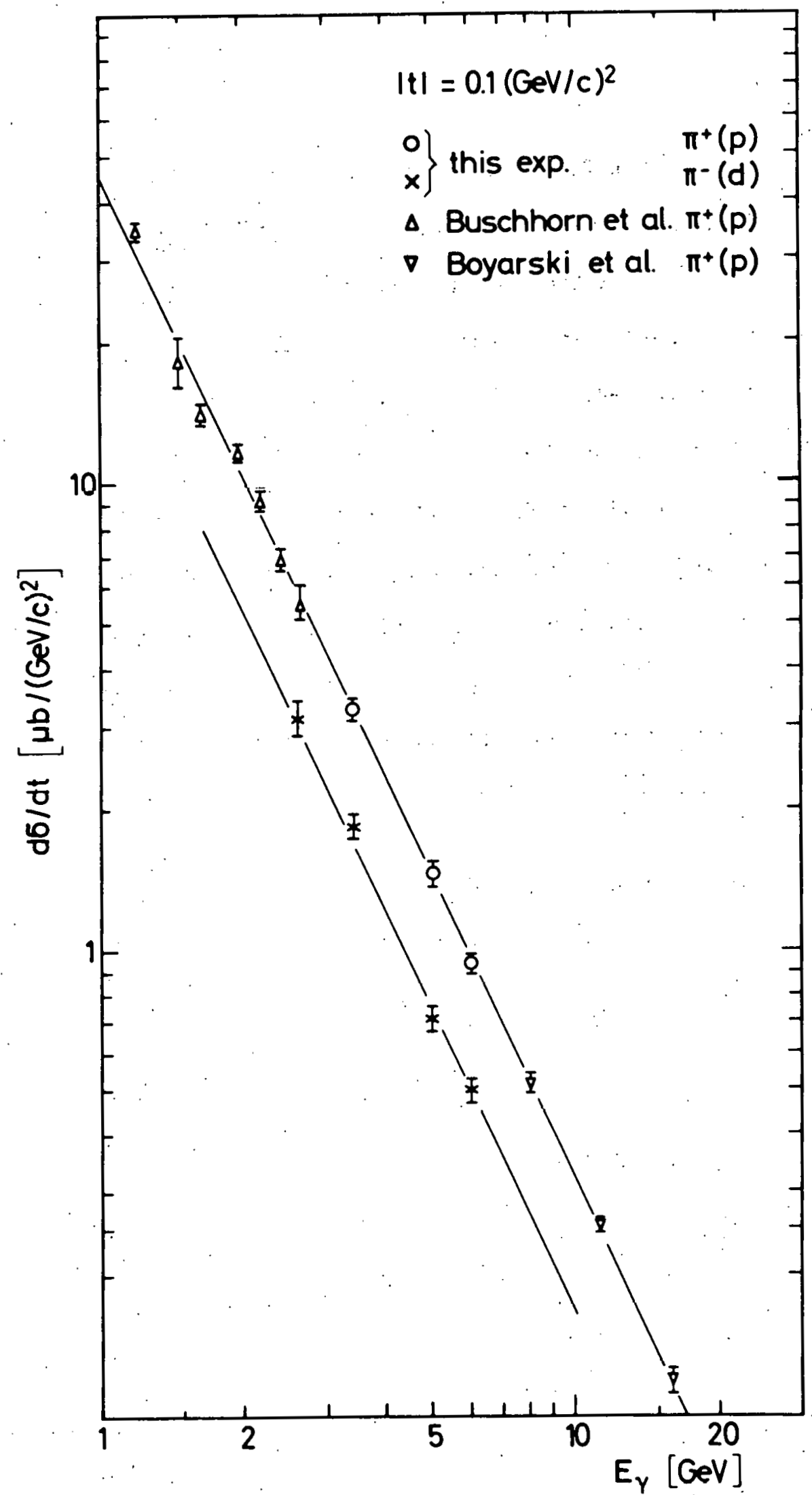

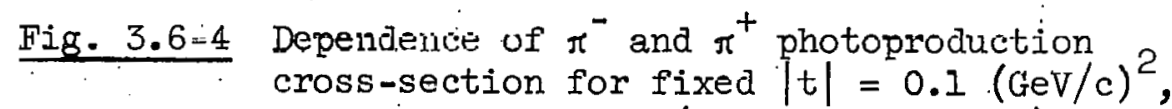
on photon energy. (From Ref. No. 88) 
A successful model for single-charged-r photoproduction will have to reproduce this behavior as well as the differential cross-section, plus any data on polarization parameters that may show up. At the time of the writing of these notes, first results are being presented for the asymetry parameter $\quad \Sigma=\frac{\sigma_{i}-\sigma_{11}}{\sigma_{\perp}+\sigma_{11}}$, defined in section 1.3 , at high energies from a $\pi^{+1}$ production experiment ${ }^{103)}$. They strongly indicate dominance of the exchange of a natural-parity object $\left(0^{+}, 1^{-}, \ldots\right)$; since the $\pi^{-} / \pi^{+}$ratio data postulate the interference of even and odd G-parity exchange at $|t|$ values not too close to zero, Fróyland and Gordon $(104)$ chose a model incorporating $\pi$ and $\rho$ exchange; a $\rho P$ and a conspiring $\pi \ddot{P}$ cut were additionally needed for a reasonable fit to the data for $\frac{d \sigma}{d t}, \Sigma$ and $R$.

\subsection{A Few Tests of SU(3) Symmetry.}

The high-energy behavior of amplitudes which are governed by exchange diagrams should provide good tests for symmetry schemes, since we expect kinematical correctionto become less important as $s$ and $t$ increase.

We mentioned in Section 1.1 that the conventional assignment of the photon as the $U=0$ part of an octet is not entirely established. This assignment stems from the Gell-Mann Nishijima formula $Q=\frac{Y}{2}+I_{3}$, which is obeyed by all known hadrons.

There is no reason (89) why there should not be an additional additive quantum number $D$ which may not transform like a member of an octet (remember that $Y$ and $I_{3}$ can be identified with two of the 
generators of the group SU(3)), so that

$$
Q=\frac{Y}{2}+I_{3}+D
$$

may contain a non-octet part. However, since for all observed hadrons $D=0$, we will keep in mind that only the octet part of $Q$ will have non-vanishing matrix elements between known hadrons. Similarly, it is possible that the relation between charge $Q$ and electromagnetic current $j$

$$
Q=\int j_{0}(\vec{x}, t) d^{3} \vec{x}(\underbrace{+\int_{1}^{3} \vec{x}}_{=0})
$$

contains an octet current $j_{0}$ whose space integral is equal to the charge, plus a conjectured additional current which may transform like a member of $\underset{\sim}{1}$ or 27 but whose space integral happens to vanish. Since the symmetry properties of the photon are those of the . electromagnetic current $j_{\mathrm{em}}$, we will have to look for possible nonoctet parts of the photon in order to establish the initial assignments. The difficulty in this search is that mogt predictiono made on the grounds of the $U=0,(\underset{\sim}{)}$ )assignment can be obtained from the U-scalar character of the photon alone. However, $\underset{\sim}{1}, \underset{\sim}{8}$ and $\underset{\sim}{27}$ all contain a U spin singlet.

Among the many $\mathrm{SU}(3)$ tests proposed, $(90)$ a few are sensitive to the octet assignment. This holds particularly for the vector dominance graph for the process $\mathrm{V}^{\circ} \rightarrow \mathrm{e}^{+} \mathrm{e}^{-}$ 


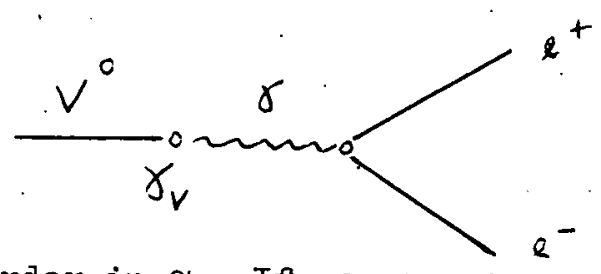

to lowest order in $\alpha$. If we assume pure octet character for the photon, then we obtain (cf. section 2.5), with the usual $\Phi-\omega$ mixing,

$$
\gamma_{\rho}^{2}: \gamma_{\omega}^{2}: \gamma_{\Phi}^{2}=9: 1: 2
$$

and, by virtue of eq. $2.5-4$ and ref. 50 ,

$$
\Gamma\left(\rho \rightarrow \mathrm{e}^{+} \mathrm{e}^{-}\right): \Gamma\left(\omega \rightarrow \mathrm{e}^{+} \mathrm{e}^{-}\right): \Gamma\left(\Phi \rightarrow \mathrm{e}^{+} \mathrm{e}^{-}\right) \approx 9: 0.65: 1.30
$$

where the neglected small terms in (2.5-4) may account for some discrepancy. The numerical check is, according to Table I,

$$
\Gamma_{\rho}=6.4 \mathrm{keV}, \Gamma_{\omega}=0.5 \text { lo } 1.1 \mathrm{keV}, \Gamma_{\Phi}=1.2 \mathrm{keV} \text {. This check is }
$$
not quantitatively tight enough to exclude some non-octet admixture for the photon, but the picture certainly looks consistent for the octet assignment.

$$
\text { Harari }(90) \text { has listed a large number of SU(3) tests in photo- }
$$
production, involving the U-scalarity of the photon. Predictions are usually for amplitudes, and high-energy experiments mostly yield cross-sections only. In the absence of phase information, we can then test inequalities rather than equalities. Assuming unbroken SU(3) symmetry, we can obtain two such inequalities for the photoproduction of pseudoscalar mesons off protons, $\sigma(\mathrm{PS}, \mathrm{B})$ :

$$
\sqrt{2} \sigma^{1 / 2}\left(\pi^{+} \mathrm{n}\right) \leq \sqrt{3} \sigma^{1 / 2}\left(\mathrm{~K}^{+} \Lambda\right)+\sigma^{1 / 2}\left(\mathrm{~K}^{+} \Sigma\right)
$$




$$
\sigma^{1 / 2}\left(\pi^{o} p\right) \leq \sqrt{2} \sigma^{1 / 2}\left(K^{o} \Sigma^{+}\right)+\sqrt{3} \sigma^{1 / 2}(\eta p)
$$

We can write eq. 3.7-4 in terms of the triangle inequality given in Fig. 3.7-1 where $\Phi$ is the unknown phase angle between the $K \Lambda$ and $K \Sigma$ amplitudes. Then SU(3) demands $|\cos \Phi| \leq 1$. Fig. 3.7-2 shows a check of the inequality $3.7-4$ by Elings et al. (91), performed at energies $3.4 \leq \mathrm{k}<4 \mathrm{GeV}$. Obviousiy $\sigma\left(\pi^{+} n\right)$, is well contained within the allowed band.

More recently, the high-energy SIAC data ${ }^{(84)}$ on forward $\mathrm{K}^{+}$ production have permitted a check on the same relations. Fig. 3.7-3 gives the results at energies $5 \leq \mathrm{k} \leq 10 \mathrm{GeV}$, and at momentum transfers $|t|$ up to $\sim 1.3(\mathrm{GeV} / \mathrm{c})^{2}$. Again, there is good agreement at $|t|$ values larger than $0.1(\mathrm{GeV} / \mathrm{c})^{2}$. At smaller $|t|$, there appears to be a strong violation of the condition $|\cos \Phi| \leq 1$. Although no precise account for this discrepancy can be given, the unequal masses involved in the processes will no doubt manifest themselves more strongly at small $|t|$ than at larger $|t|$ values.

The relation 3.7-5 cannot at present be checked since there are no good data available for $\gamma \mathrm{p} \rightarrow \mathrm{K}^{\circ} \Sigma^{+}$. Predictions involving reactions with more than two particles in the final state are not well-enough studied experimentally to provide stringent tests.

\subsection{High-Energy $\eta$ Photoproduction.}

In section 1.5, we mentioned the usefulness of $\eta$ production in the isobar region as a probe for the $I=1 / 2$ channel. In the tramework of higher energy production t-channel exchange processes of 


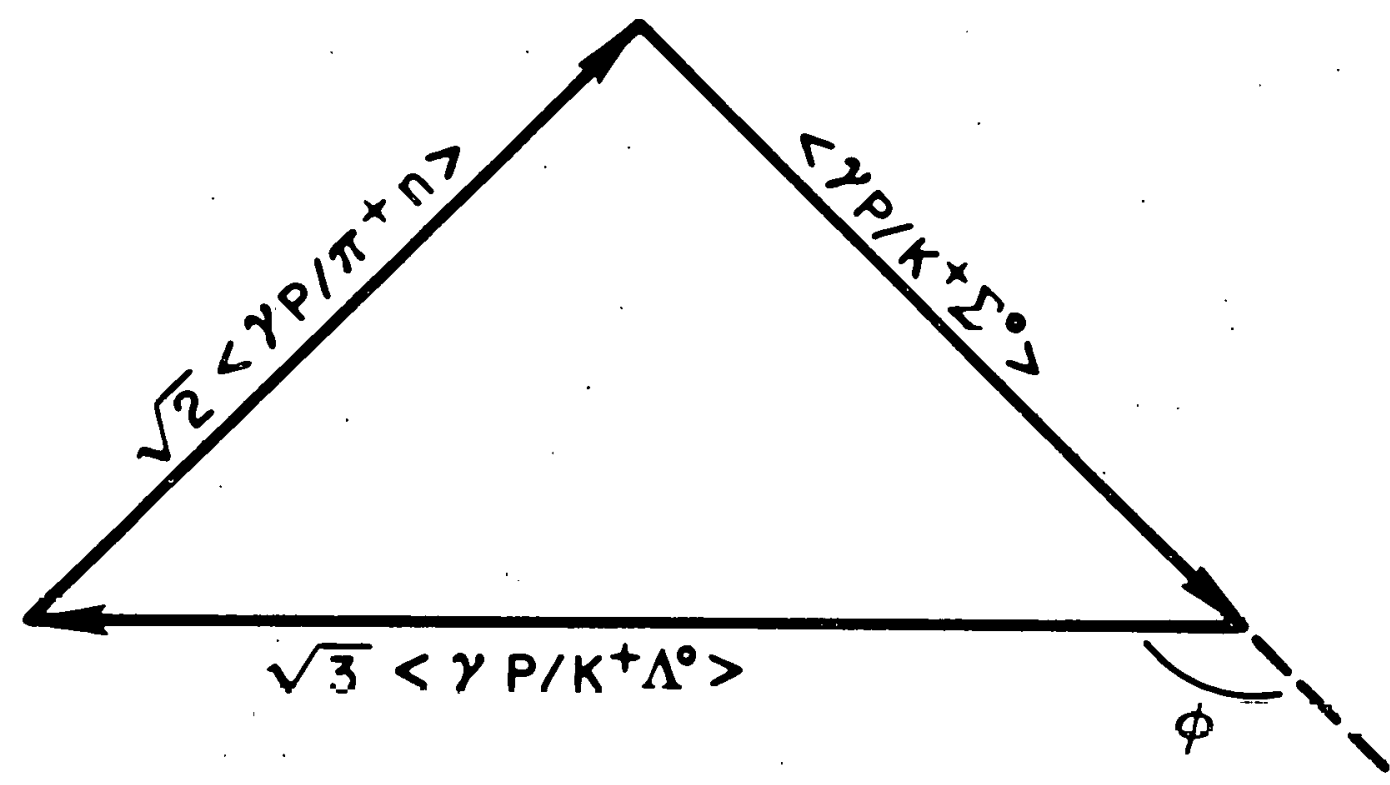

Fig. 3.7-1 SU(3) relation between photoproduction processes $\left(\pi^{+} n\right),\left(\mathrm{K}^{+} \Sigma^{\mathrm{O}}\right)$ and $\mathrm{K}^{+} \Lambda^{\odot}$. (From Ref. No. 9l)

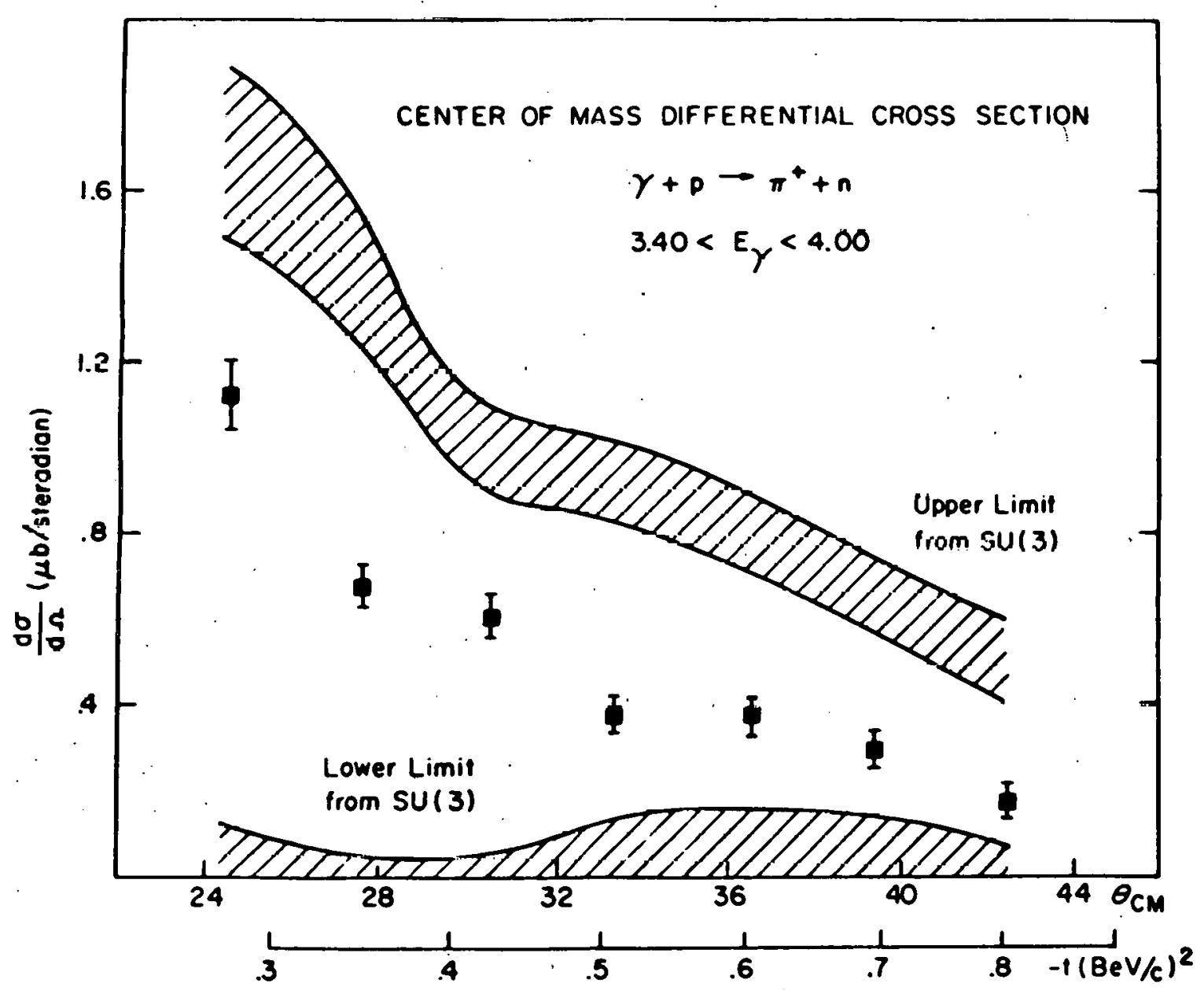

Fig. 3.7-2 Comparison of the experimental values for $\gamma p \rightarrow \pi^{+} n$ with the SU(3) limits imposed by the inequality 3.7-4 and Fig. 3.7-1. (From Ref. No. 91) 


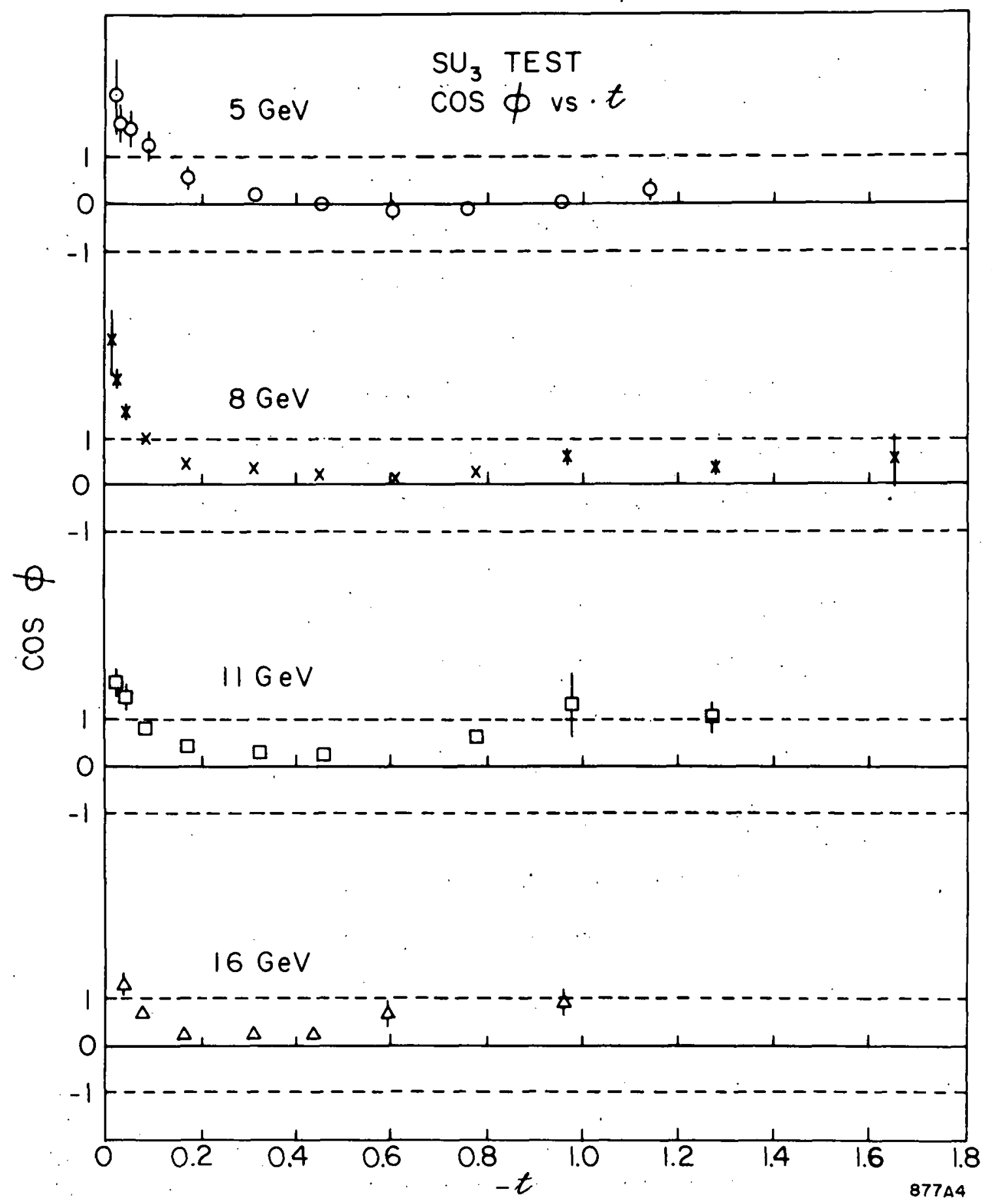

Fig. 3.7-3 SU(3) check according to ed. 3.7-4, as a functionn of momentum transfer $|t|$. The $S U(3)$ condition $|\cos \Phi|<1$ is violated at small $|t|$. (From Ref. No. 84) 
the type

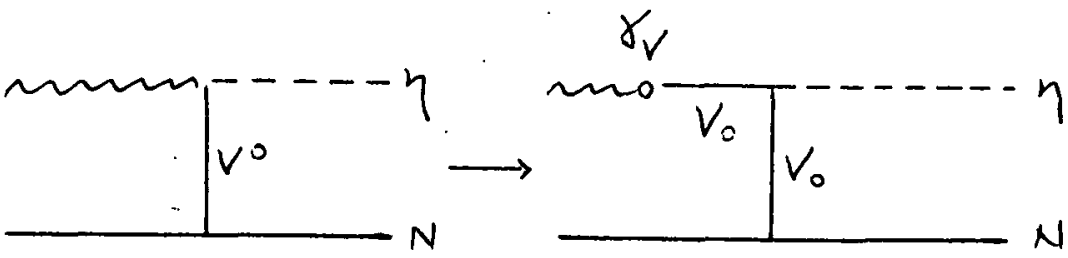

are expected to play a dominant role in analogy to the $\pi$ production processes. If we invoke the vector dominance model, we have two strong vertices to deal with, and can make statemenț ștemming from isospin and SU(3) invariance.

On such grounds, Dar and Weisskopf ${ }^{(92)}$ made predictions for the $t$ dependence of the differential cross-section $\frac{d \sigma}{d t}(\gamma p \rightarrow p \eta)$ at higher energies. Note that, in this case, the prediction came before the experiment!

One can safely assume ${ }^{(92)}$ that, in the above diagram, $\rho$ exchange dominates the amplitude. Connecting the respective coupling constants through SU(6) relations and isospin invariance, one can link up the $\rho$ exchange amplitudes in $\gamma p \rightarrow p \eta$ and $\gamma p \rightarrow \pi^{+} n$. The vector dominance model then relates the $\rho$ exchange amplitude for $\gamma p \rightarrow \pi^{+} n$ to the $\rho$ exchange amplitude for $\omega p \rightarrow \pi^{+} n$. Note that for reasons of G-parity conservation, we can have only the $\omega$ as the intermediate vector meson in the graph

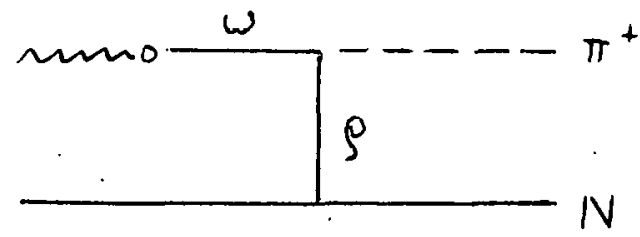


Finally, we invoke time-reversal invariance, and projecting out the transversely polarized $\omega^{\prime}$ s only, we arrive at the relation

$$
\frac{d \sigma}{d t}(\gamma p \rightarrow p \eta) \approx \frac{3.3}{2} \frac{\pi \alpha}{\gamma \omega^{2}}: P_{11} \frac{d \sigma}{d t}\left(\pi^{+} n \rightarrow \omega p\right)
$$

where the numerical factor is due to the SU(6) coupling ratios, and to the assumption of an unpolarized photon beam; $\gamma_{\dot{\omega}}$ denotes the strength for the direct coupling $\gamma-\omega$, as introduced in section 2.5 .

Since, by isospin invariance, we can replace $\pi^{+} \mathrm{n} \rightarrow$ wp by $\pi^{-} \mathrm{p} \rightarrow \omega_{n}$, we can draw on the data for both these reactions to predict the $\gamma p \rightarrow p \eta$ cross-section at given energies. This is done in Fig. 3.8-I, which gives the quantity $s^{2} \frac{d \sigma}{d t}$ vs. the momentum transfer $t$, for incident energies $\sim 3$ and $10 \mathrm{GeV}$.

Note that this prediction does not show either a forward dip or a secondary dip around $-t \approx 0.5(\mathrm{GeV} / \mathrm{c})^{2}$. Both these features are present in $\pi^{\circ}$ production (at least at intermediate energies), and would be expected here if we assumed simple $\rho$ trajectory exchange (cf. section 3.3 ).

First data were recently taken at $\mathrm{CEA}{ }^{(93)}$ at $\mathrm{k}=4 \mathrm{GeV}$ and at SLAC ${ }^{(40)}$ at $k=6 \mathrm{GeV}$. The $6 \mathrm{GeV}$ data are shown in Fig. 3.8-2. The general trend appears to bear out the prediction; the dip structure observed in $\pi^{n}$ production is not seen here. The measurements were taken at $|t|>0.2(\mathrm{GeV} / \mathrm{c})^{2}$, so that no statement can be made about a forward dip. Quantitatively, there is agreement within a factor of 2. This ought to be regarded as reasonable agreement, considering the approximations made and the errur bars on lhe eruss-seclluns. 


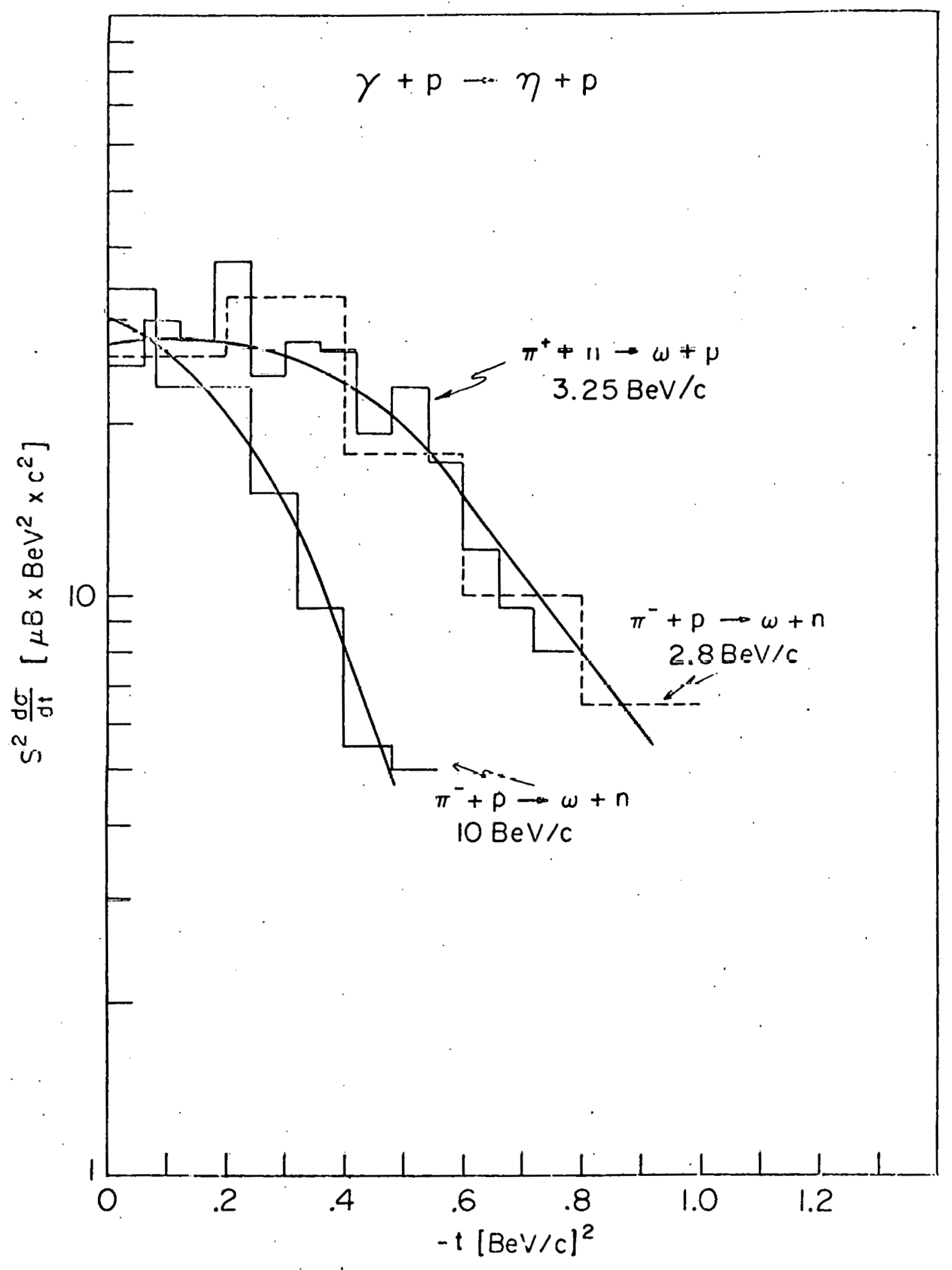

Fig. 3.8-1 Prediction for high-energy $\eta$ photoproduction cross-section according to eq. 3.8-1, using the vector dominance model and $\pi \mathbb{N} \rightarrow$ wN data. (From Ref. No. 92) 


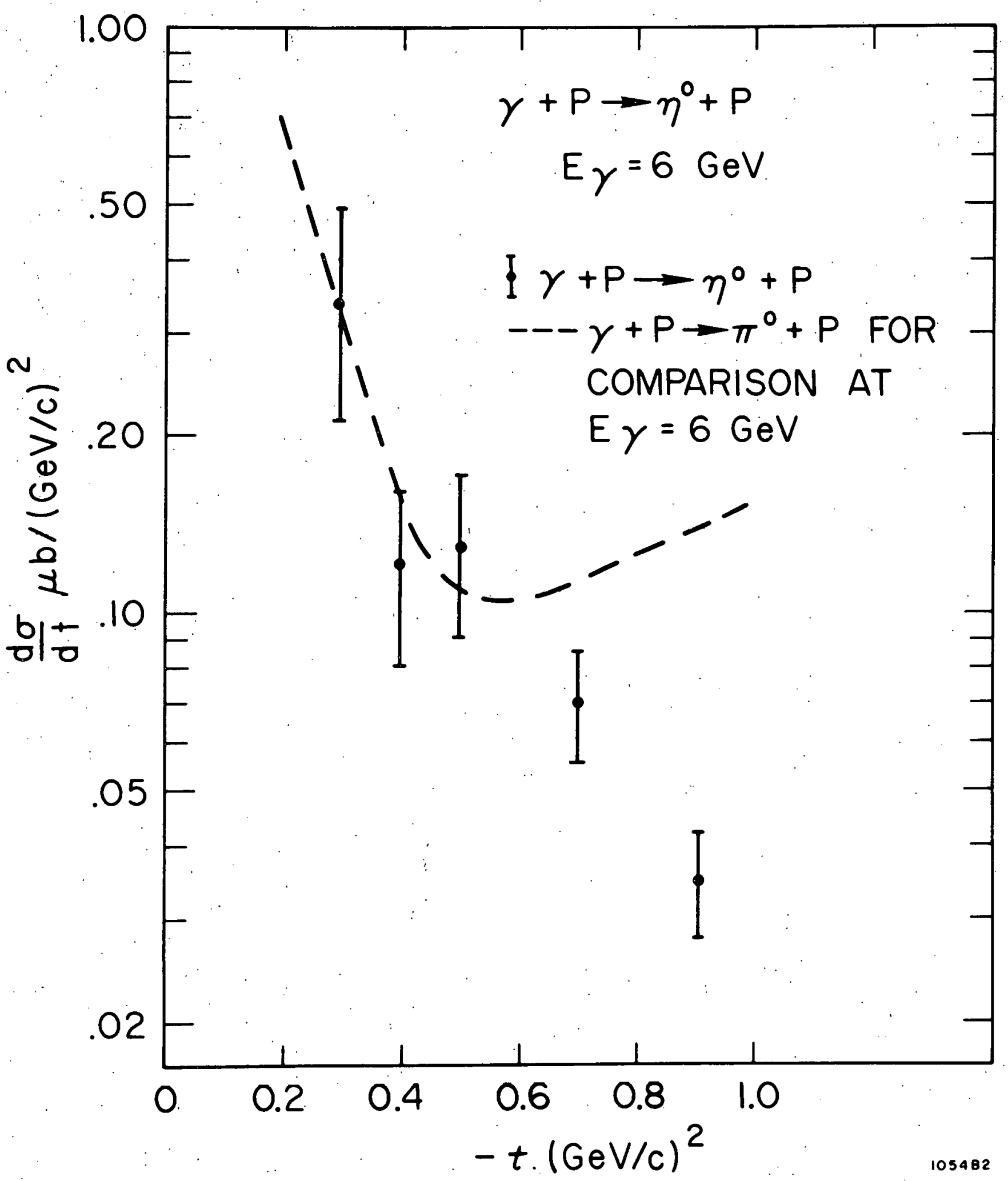

Fig. 3.8-2 Differential cross-section for high-energy $\eta$ photoproduction: Dashed curve: general behavior of $\pi^{\circ}$ photoproduction crosssection in comparable region. (From Ref. No. 40) 
The preliminary CEA data do go to smaller $t$, and may contain indications for a dip at $|t|<0.5(\mathrm{GeV} / \mathrm{c})^{2}$. At larger values, they also agree qualitatively with the predictions ${ }^{(92)}$. Clearly, we will have to wait for more data before we can do a detailed analysis.

\subsection{Concluding Remarks}

In the preceding argumentation, we are left with a slightly uneasy feeling. We start with a simple $\rho$ exchange picture which would make us expect a dip-and-peak angular structure somewhat like in the $\pi^{\circ}$ case, Fig. 3.3-1. Invoking several reasonably well established invariance principles and models, we wind up with a predicted cross-section quite unlike the typical one-trajectory exchange picture of our "input". Maybe this points up a situation typical for the "state of the art". The theory is a curious blend of pragmatism, based on suggestive principles plus ad-hoc assumptions which may or may not appear compelling; they may carry little immediate intuitive appeal, and lack the solid basis to satisfy the more rigorous theorist.

Experiment is found, within reasonable error, to bear out some of the features we set out to describe - but the limited range of parameters measured, and the need for more precise data leave agreement in a doubtful state.

On this open-ended note we will conclude these lectures. They may, altogether, indicate that photon-induced hadronic reactions are particularly suited to study distinctive features of the strong interactions; that photon experimentation, which carries its own 
difficulties, has come a long way in recent years; that theoretical understanding is in no better and no: worse shape here than in the general field of hadron-induced hadronic interactions; and that physicists in this field will not be on the unemployed roll for some time to come.

A final remark appears in order on what important experimental dcrclowmenl wan we anticipated in the field of photon-hadron physics in the near future. In the course of these lectures, we have stressed time and again that the need for the fixing of photon momentum and polarization parameters, and the need to check truly asymptotic behavior, leave present interpretation of the data open to doubt.

The production of polarized photon beams is just now becoming practically feasible, along several lines. At DESY and CEA, coherent bremsstrahlung production off. crystals ${ }^{(95)}$ is yielding photon beams of good intensity ( $\lesssim 10^{11}$ equivalent quanta/min) with a high degree of plane polarization $(\lesssim 80 \%)$, at energies up to about one-half of the final machine energy (1.e. $\lesssim 3.6 \mathrm{GeV}$ at DESY). The cross-sections for two-body reactions can be broken up into parts with only natural or unnatural parity exchanges $(95)$, so that these beams will help greatly in the understanding of exchange processes. One simply has to study the distribution

$$
\frac{d \sigma(\Phi)}{d \Phi}=A+B \cos ^{2} \Phi+C \sin ^{2} \Phi
$$

where $\Phi$ is the angle between the photon polarization and the reaction 
plane, and the successive terms on the RHS indicate the contributions from unpolarized photons, from natural $\left(0^{+}, 1^{-}, \ldots\right)$, and from unnatural $\left(0^{-}, 1^{+}, \ldots\right)$ parity exchanges. In the concluding remarks of section 3.6, we saw that first results from such data in $\pi^{+}$ photoproduction put an important constraint on the models used to describc the prooese.

Monochromatic beame from positron annihilation ${ }^{(06)}$ and f'rom backscattered laser photons ${ }^{(97)}$ are in initial use at SLAC with intensities useful for bubble chamber experimentation. The laser photons can carry circular as well as plane polarization, and the. attractive feature of this scheme is that $180^{\circ}$ scatters preserve this polarization fully.

Lastly, it should be mentioned that even in the absence of plans to build higher-energy electron machines, photon experimentation at energies more closely satistying asymptot1c cond1tiuns will become one of the standard teatures at the $200-500$ leV proton accelerators now being projected at Weston and Saint-Trope * ${ }^{*}$. Fig.5 $3.9-1$ and $3.9-2$ give rough estimates ${ }^{(98)}$ of the photon yield per interacting proton, per BeV energy interval, from pp collisions at $200 \mathrm{BeV}$. Intensities are lower but still respectable even for purified beams $(98)$, and together with the good duty-cycle cluaracteristics of the proton machines, and large-solid-angle detection devices, make us look forward to the solution of some presently unanswerable questions.

* The error bars on this last statement presently reach from the Escorial to somewhere in Northern Sweden. 


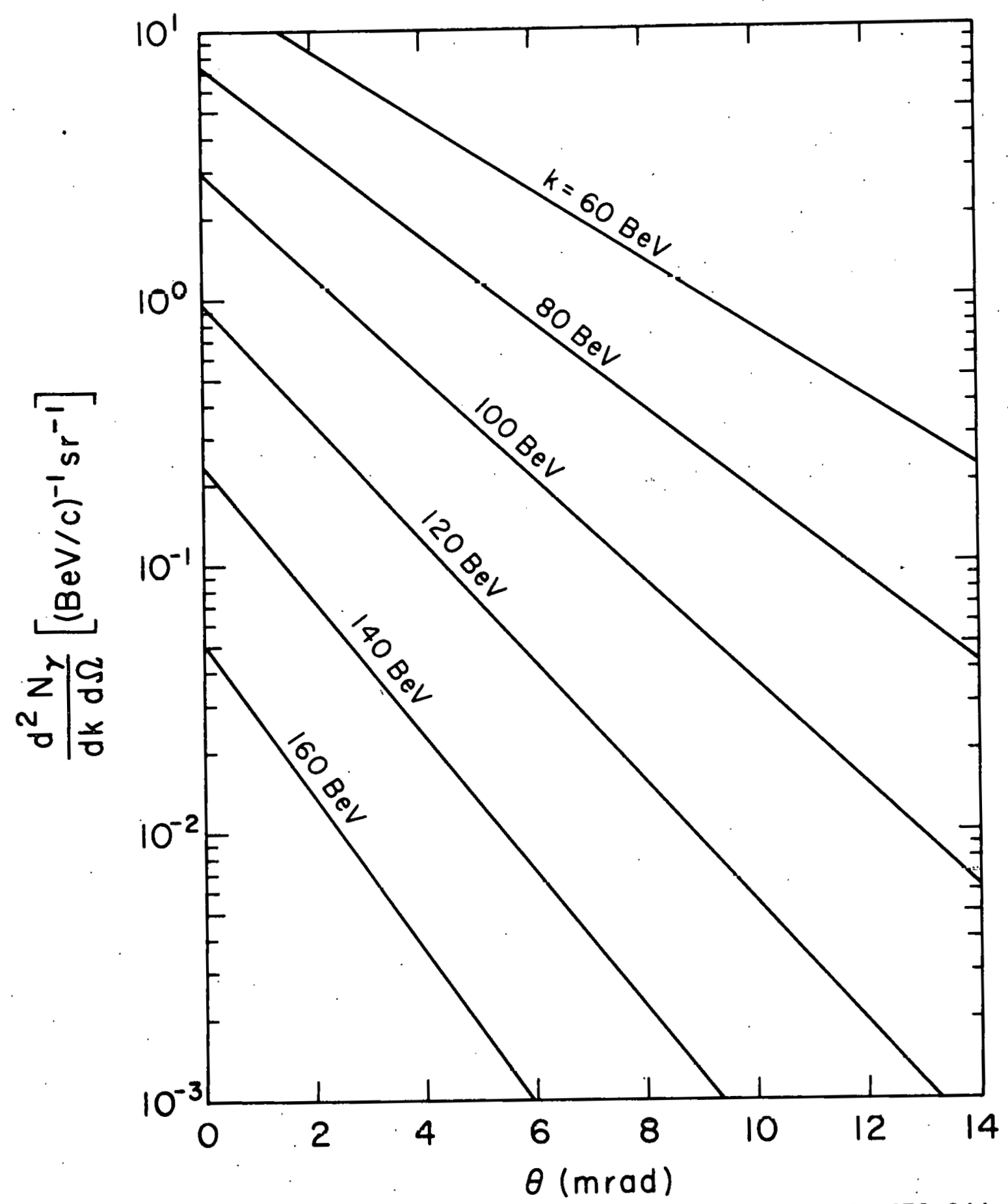

XBL672-844

Fig. 3.9-1 Angular dependence of photon yield per unit energy interval; per $200 \mathrm{GeV}$ protun interacting with a proton target at rest. (From Ref. No. 98) 


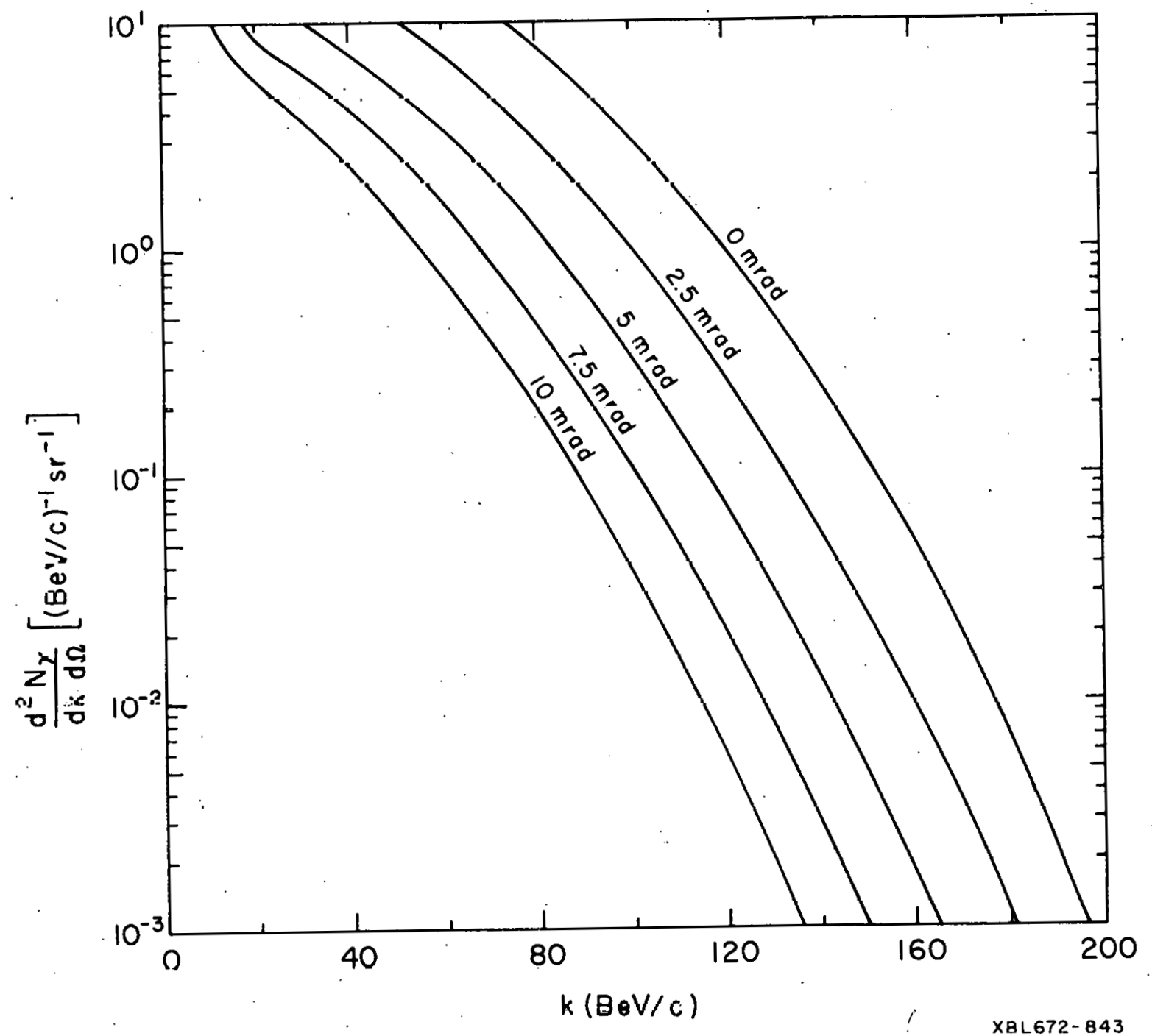

Fig. 3.3-2 Estimate of photon yield per unit energy, per interacting $200 \mathrm{GeV}$ proton, as a function of photon energy, for various angles. (From Ref. No. 98) 
Acknowledgments

I would like to thank many colleagues for informative discussions. In particular, I am grateful to experimenters at SLAC, DESY, and CEA who made their results available to me prior to publication. I appreciate the hospitality of the Aspen Center for Physics, where these notes were written up. Drs. Stephen Adler, Leon Rochester, and Karl Strauch gave them a critical reading, for which I am grateful. 


\section{$\underline{\text { References }}$}

I) M. J. Moravcsik, P.R. 104, 1451 (1956).

2) M. Gourdin, P. Salin, N.C. 27, 193 (1963).

3) P. Salin, Nv.C. 2ㅇ, 1294 (1963).

4) D. Beder, N.C. 33, 94 (1964).

5) R. L. Walker, submitted to P.R. (1968).

6) M. Jacob and G.C. Wick, Ann. Phys. 7, 404 (1959).

7) See the data compilation of J. T. Beale, S. D. Ecklund,

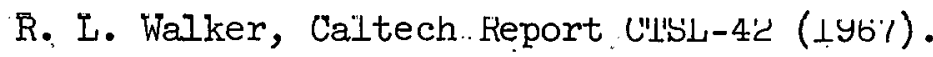

8) M. Borghini, CERN preprint; Contribution to Dubna Conf. (1967).

9) G. F. Chew, M. L. Goldberger, F. E. Low, and Y. Nambu, P. R. 106,1345 (1967).

10) S. D. Ecklund and R. L. Walker, P. R. 159, 1195 (1967).

11) E. D. Bloom, C. A. Heusch, C. Y. Prescott, L. S. Rochester, F.R.L. 19, 071 (1067).

12) H. Harari, Summary talk, Proc. SLAC Symposium (1967).

13) N. Cabibbo, L. Radicati, P.I. 19, 697 (1966).

14) F. J. Gilman, H. J. Schnitzer, P.R. 150, 1362 (1966).

15) S. D. Drell, A. G. Hearn, P.R.L. 16, 908 (1966).

16) Y. C. Chau, R. C. Moorhouse, N. Dombey, P.R. 163, 1632 (1967), and contribution to SLAC Symposium(1967).

17) C. A. Heusch, Lecture at the 1966 Erice Summer School.

18) E. D. Bloom, C. A. Heusch, C. Y. Prescott, L. S. Rochester, P.R.L. (to be published).

19) German Bubble Chamber Collaboration, DESY 68/8 (1968).

20) S. D. Deans, W. G. Holladay, P.R. 161, 1466 (1967).

21) C. A. Heusch, C. Y. Prescott, E.D. Bloom, I. S. Rochester, P.R.L. 17, 573 (1966). 
22) C. A. Heusch, C. Y. Prescott, R. F. Dashen, P.R.L. 17, 1019 $(1966)$

23) J. Tyson, et al., quoted by K. Strauch, Proc. SLAC Symposium (1967).

24) H. Rollnik, Proc. Heidelberg International Conference on Elementary Particles, (1967); Lectures at Int. School of Physics, Herceg-Novi (1967).

25) J. S. Ball, P. R. 114, 2014 (1961).

26) R. Taylor, private communication.

27) R. Dalitz, Lectures at Grenoble Summer School, 1965.

28) R. Moorhouse, P.R.L. 16, 772 (1966).

29) S. D. Drell, Proc. SLAC Symposium (1967), p.

30) M. Ross, L. Stodolsky, P.R.I. 149, 1172 (1966); see also

S. D. Drell and J. Trefil, P.R.I. 16, 552 (1966).

31) P. Söding, P.I. 19, 702 (1965).

32) V. Auslander et al., Novosibirsk preprint (1967).

33) J. Pisút, M. Koo's, Nuc. Phys. B6, 325 (1968).

34) Y. Eisenberg et a.1., P.I. 22, 223 (1966).

35) G. Kramer, DFGY $67 / 32$ (1967).

36) M. Davier, et al., SLAC preprint (1968).

37) H. Blechschmidt, et al., DESY 67-30 (1967).

38) J. G. Asbury, et al., P.R.I. 19, 865 (1967).

39) J. Fleischer, DESY 68/15 (1968).

40) W. G. Jones, et al., SLAC preprint (1968); and R. Anderson, et al., SLAC-PUB-431 (1968).

41) K. Schilling and F. Storim, DESY 68/23 (1968). 
42) D.R.O. Morrison, P.I. 22, 528 (1966)

43) Preliminary results, quoted by S.C.C. Ting (1968).

44) Y.S. Tsai et al., P.R.L. 19, 915 (1967).

45) A. Boyarski et al., P.R.L. 20, 877 (1968).

46) S. Meshkov and R. Ponzini, National Bureau of Standards preprint (1968).

47) For a list of references, see H. Joos, Proc. Heidelberg Conference (1.967)

48) S. C.C. Ting, private communication (based on $\sim 50 \Phi \rightarrow e^{+} e^{-}$ events (1967).

49) A. Zichichi, private communication (based on $~ 10$ events for both $\omega$ and $\Phi)$, (1968).

50) R. J. Oakes and J. J. Sakurai, P.R.I. 19, 1266 (1967).

51) K. Kajantje and J.S. Trefil, CERN Th. 736 (1966).

52) A. Dar et a.., P.R.I. 20, 1261 (1968).

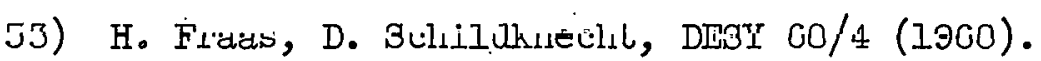

54) R. Diebold and J. A. Poirier, P.R.L. (SLAC-PUB-412 (1968)).

55) M. Krammer, DESY 68/6 (1968).

56) J. S. As.bury et al., P.R.I. 20, 227 (1968).

57) H. Joos, D. Schildknecht, DESY preprint (1968).

58) I. Etudululsy, P. R.L. 18, 135 (1867).

59) H. G. Hilpert et al., DESY preprint (1968).

60) L. N. Hand, P.R. 129, 1834 (1963).

61) L. W. Mo, Y. S. Tsai, SLAC-PUB-380 (1968).

62): M. Braunschweig, et al., Boun preprint 1-038 (1968). 
63) G. C. Bolon et a.1., P.R.L. 18, 926 (1967).

64) G. Buschhorn et al., P.I. 25B, 622, (1967).

65) G. Buschhom et al., P.R.I. 18, 571 (1967).

66) P. M. Joseph, et al., Contribution to the SLAC Symposium (1967); P.R.L. 19, 1206 (1967).

67) A. M. Boyarski, et al., P.R.I. 20, 300, (1968).

68) A. M. Boyarski, et al., quoted by B. Richter, Proc. SLAC Symposium (1967).

69) G. Buschhorn et al., P.R.L. 20, 230 (1968).

70) B. Gittelman, private communication (1968).

71) R. Anderson et al., SLAC-PUB-428 (1968).

72) M. P. Locher, H. Rollnik, P.I. 22, 696 (1966).

73) J. P. Ader, M. Capdeville, P. Salin, CERN Th. 803 (1967).

74) P. Stichel, Lectures on Photoproduction, DESY 66/2 (1966).

75) L. S. Osborne, private communication (1968).

76) M. Ross, private communication (1968).

77) J. Orear, et al., P. R. 152, 1162 (1966); also J. Orear, Proc. of the Topical Cunference on Hadrons, CERN, Geneva (1968).

78) E. A. Paschos, SLAC preprint (1968).

79) H. Abarbanel et al., P. R. 160, 1329 (1967).

80) R. F. Dashen, S. C. Frautschi, P. R. 143, 1171 (1966),

8I) H. R. Rubenstein et al., P. R. 167, 144I (1967).

82) S. Mandelstam, Wang, P. R. 160, 1490 (1967).

83) Lorella Jones, Caltech internal note (1968).

84) B. Richter, Proc. SLAC Symposium (1967); SLAC-PUB- 353 (1967). 
85) J. S. Ball et al., P.R.L. 20, 518 (1968), see also

A. Bietti et al., P. L. 26B, 457 (1968).

86) D. Amati et.al., P. L. 26B, 510 (1968).

87) Z. Bar-Yam et al., P.R.L. 19; 40 (1967).

88) P. Heide et al., DESY 68/22 (1968).

89) M. Nauenberg, P.R. 135 , B. 1047 (1964).

90) H. Harari, F.R. 155, 1565 (1967); cf. also H. Harari, SLAC-PUB-349 (1967).

91) V. Elings et al., P.R.L. 16, 474 (1966).

92) A. Dar, V. F. Weisskopf, P.R.L. 20, 762 (1968).

93) D. Belenger et al., M.I.T. preprint (1968).

94) L. Criegee et a.., P.R.L. 16, 1031 (1966), and references there.

95) J. P. Ader et al., CERN Th. 886 (1968).

96) G. Chadwick, Note in SLAC Handbook (1967).

97) T. T. Murray, P. R. KDein, SLAC-TN-67-19 (1967), and references there,

98) C. A. Heusch, $200 \mathrm{BeV}$ Accelcrator, Studies on Experimental Use, p. 156 UCRL 16830 (1967).

99) C. Bacci et al., P.R.I. 20, 571 (1968)

100) A. Donnachie, P.I. 24B, 420 (1967).

107) Orsay Contribution to the Vienna Conference (1968).

102) I. Derado, Z.G.T. Guiragossian, SLAC-PUB-460 (1968).

103) C. Geweniger et al., Contribution to the Vienna Conference (1968).

104) J. Froyland, D. Gordon, M.I.T. preprint CTP38 (1968). 
LEGAL NOTICE

This report was prepared as an account of Government sponsored work. Neither the United States, nor the Commission, nor any person acting on behalf of the comisstion:

A. Makes any warranty or representation, expressed or implied, with respect to the accuracy,-completeness, or usefulness of the information contalned in this report, or that the use of any information, apparatus, method, or process disclosed in this report may not infringe privately owned rights; or

B. Assumes any liabilities with respect to the use of, or for damages resulting from the use of any information, apparatus, method, or process disclosed in this report.

As used in the above, "person acting on behalf of the Commission" includes any employee or contractor of the commission, or employee of such contractor, to the extent that such employee or contractor of the Commission, or employee of such contractor prepares, disseminates, or provides access to, any information pursuant to his employment or contract with the Comission, or his employment with such contractor. 